San Jose State University

SJSU ScholarWorks

Master's Theses

Master's Theses and Graduate Research

Summer 2020

\title{
LC-MS/MS Method Development for the Quantitation of \\ Methylmalonic Acid Using Aqueous Normal Phase \\ Chromatography
}

Daniel Joseph Biocini

San Jose State University

Follow this and additional works at: https://scholarworks.sjsu.edu/etd_theses

\section{Recommended Citation}

Biocini, Daniel Joseph, "LC-MS/MS Method Development for the Quantitation of Methylmalonic Acid Using Aqueous Normal Phase Chromatography" (2020). Master's Theses. 5118.

DOI: https://doi.org/10.31979/etd.r2rd-jan9

https://scholarworks.sjsu.edu/etd_theses/5118

This Thesis is brought to you for free and open access by the Master's Theses and Graduate Research at SJSU ScholarWorks. It has been accepted for inclusion in Master's Theses by an authorized administrator of SJSU ScholarWorks. For more information, please contact scholarworks@sjsu.edu. 


\title{
LC-MS/MS METHOD DEVELOPMENT FOR THE QUANTITATION OF METHYLMALONIC ACID USING AQUEOUS NORMAL PHASE CHROMATOGRAPHY
}

\author{
A Thesis \\ Presented to \\ The Faculty of the Department of Chemistry \\ San José State University \\ In Partial Fulfillment \\ of the Requirements for the Degree \\ Master of Science
}

by

Daniel Joseph Biocini

August 2020 
(C) 2020

Daniel Joseph Biocini

ALL RIGHTS RESERVED 
The Designated Thesis Committee Approves the Thesis Titled

LC-MS/MS METHOD DEVELOPMENT FOR THE QUANTITATION OF METHYLMALONIC ACID USING AQUEOUS NORMAL PHASE CHROMATOGRAPHY

by

Daniel Joseph Biocini, MT(ASCP)

APPROVED FOR THE DEPARTMENT OF CHEMISTRY

SAN JOSÉ STATE UNIVERSITY

August 2020

Daryl Eggers, Ph.D. Department of Chemistry

Laura Miller-Conrad, Ph.D. Department of Chemistry

Joseph Pesek, Ph.D. Department of Chemistry,

Professor Emeritus 


\section{ABSTRACT \\ LC-MS/MS METHOD DEVELOPMENT FOR THE QUANTITATION OF METHYLMALONIC ACID USING AQUEOUS NORMAL PHASE CHROMATOGRAPHY}

by Daniel Joseph Biocini

The aim of the study was to develop a quantitative assay for methylmalonic acid (MMA), an important biomarker for clinical diagnosis of vitamin B12 (B12) deficiency. The techniques of liquid chromatography and tandem mass spectrometry (LC-MS/MS) are used for separation and quantitation of MMA, as well as its endogenous isomer succinic acid. The MS/MS method was developed using an infusion set-up and manual tuning experiments. Relevant transitions were detected for the two analytes and MMA-d3, then a preliminary MS/MS method was developed. The preliminary LC method was further optimized. Various gradients, mobile phases (MPs), and additive combinations were used across three-column chemistries. The three-column chemistries chosen were representative of distinct separation modes, C18 for reverse phase, Diamond Hydride (DH) for aqueous normal phase (ANP), and BEH-Amide for hydrophilic interaction liquid chromatography. The chromatographic parameters of peak shape, capacity factor $(k)$, selectivity $(\alpha)$, and resolution $(\mathrm{R})$ were used to judge the quality of separation. In conclusion, the $\mathrm{DH}$ column showed the best peak shape, $k$, and $\mathrm{R}$. The peak width of MMA on DH was 0.13 minutes with a symmetrical shape, and $\mathrm{R}_{\mathrm{MMA}}$ on DH was 4.7. In contrast, BEH-Amide showed the best $\alpha$. Additionally, experimental evidence confirmed that the DH column retains by an ANP mechanism, as shown by the increase in $k_{M M A}$ from 3.72 to 4.89 , with increased retention when using a low salt MP. 


\section{ACKNOWLEDGEMENTS}

I would like to acknowledge my thesis committee, Dr. Pesek, Dr. Eggers, and Dr. Miller-Conrad, for their support and responsiveness throughout the process. In addition, I would like to thank the Chemistry Department at SJSU for offering a program with so much freedom in course offerings and electives, which allowed me to approach this project in a way that complimented my current career path. I would like to thank the Valley Medical Center Laboratory Toxicology Department for allowing me instrument time and supplies to perform the method development experiments. There were three people in particular at Valley Medical Center that really supported my growth throughout this project: Thomas Kampfrath Ph.D., Penny Peng MT(ASCP), and Philip Sobolesky, Ph.D. I would like to thank Waters and especially Erik Todd, for the generous gift of polar columns for these experiments. Finally, and most importantly, I would like to thank my wife, who has been my support system and inspiration throughout this MS. The strength she has shown while pregnant and now raising our daughter, as I have buried my head in chemistry texts over the last few years has been nothing short of heroic. I would like to dedicate this to my daughter Lydia Noemi Biocini; I could not have finished without your smiles of encouragement. 


\section{TABLE OF CONTENTS}

List of Tables............................................................. viii

List of Figures............................................................... ix

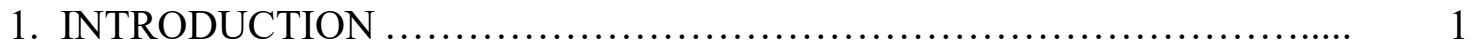

1.1 Background ...................................................... 1

1.1.1 Method Development in the Clinical Laboratory.................... 1

1.2.1 Methylmalonic Acid.......................................... 3

1.2.1.1 MMA Biochemistry .................................... 3

1.2.1.1.1 Methylmalonic Acidemia.............................. 3

1.2.1.1.2 B12 Deficiency..................................... 7

1.2.1.2 MMA Structure........................................ 11

1.2.1.3 Testing Strategies for MMA............................. 12

$\begin{array}{ll}\text { 1.3.1 Brief Review of Analytical Methods in the Clinical Laboratory..... } & 12\end{array}$

1.4.1 Chromatography.......................................... 16

1.4.1.1 History of Chromatography................................ 16

1.4.1.2 Important Concepts in Chromatography...................... 17

1.4.1.3 Van Deemter Equation...................................... 19

1.4.1.4 Modes of Separation.................................... 21

1.4.1.4.1 Reverse Phase vs. Normal Phase....................... 22

1.4.1.4.2 HILIC vs. ANP...................................... 24

1.4.1.5 Ultra High-Pressure Liquid Chromatography Instrumentation 29

1.5.1 Mass Spectrometry ........................................... 31

1.5.1.1 GC-MS ................................................. 37

1.5.1.2 LC-MS/MS .............................................. 38

1.6.1 MMA in Literature......................................... 43

1.6.1.1 GC-MS for MMA........................................ 44

1.6.1.2 LC-MS/MS for MMA.................................. 47

1.7.1 Research Goals............................................. 54

2. MATERIALS AND METHODS ............................................ 56

2.1 Materials......................................................... 56

2.1.1 Analytes.................................................... 56

2.1.2 Mobile Phase Constituents................................... 58

2.1.3 Columns..................................................... 59

2.1.4 Miscellaneous Materials......................................... $\quad 60$

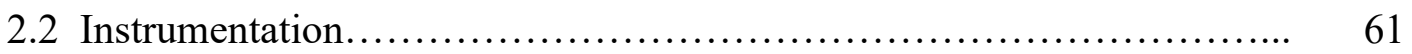

2.3 Methods......................................................... 64

2.3.1 Development of an MS/MS Method for MMA, MMA-d3, and SA* 64

2.3.1.1 Identification of Precursor Ions.......................... 65

2.3.1.2 Identification of Fragment Ions............................ 67

2.3.2 Development of an LC Method for MMA, MMA-d3, and SA*... 69 
2.3.3 Comparing Retention Modes of ANP and HILIC................ 71

3. RESULTS AND DISCUSSION ...................................... 73

3.1 Development of an MS/MS Method for MMA, MMA-d3, and SA*..... 73

3.1.1 Contamination of the Infusion Inlet......................... 74

3.1.2 Low-End Mass Accuracy Issue in ESI Negative Mode........... 78

3.1.3 Path to a Successful MS/MS Method.......................... 81

3.2 Development of an LC Method for MMA, MMA-d3 and SA*......... 87

3.2.1 Preliminary RT and Peak Shape............................ 88

3.2.2 Reproducibility - The Trends of RT and Peak Shape............. 95

3.2.3 Selectivity and Resolution ................................. 106

3.2.4 Buffer Salt Concentration to Verify ANP Retention Principles..... 112

4. CONCLUSION AND FUTURE WORK.............................. 120

5. LITERATURE CITED ............................................. 125 


\section{LIST OF TABLES}

Table 1. LC-MS/MS vs. GC-MS for MMA.............................. 46

Table 2. MMA MS/MS Values from Literature............................ 58

Table 3. Analyte Vendor Information.................................. 57

Table 4. Analyte Molecular Formulas.................................. 57

Table 5. Mobile Phase Constituents................................. 58

Table 6. Column Dimensions............................................ 60

Table 7. Additional Lab Equipment.................................... 61

Table 8. $\quad$ ESI Negative Mode - MS/MS Preliminary Method.................. 73

Table 9. MS/MS Tune Parameters...................................... 74

Table 10. Preliminary Gradient............................................ 88

Table 11. Summary of Chromatographic Results Obtained with High Salt MP... 111

Table 12. Varied MP Additive Concentration to Test ANP Retention Theory..... 119 


\section{LIST OF FIGURES}

Figure 1. Biochemical pathway of methylmalonic acid..................... 5

Figure 2. Two important B12 dependent pathways........................ 8

Figure 3. Structures of analytes...................................... 11

Figure 4. Immunoassay............................................ 13

Figure 5. Beer's Law............................................. 13

Figure 6. Partition mechanism in HILIC ............................... 25

Figure 7. Type B vs. type C silica.................................. 26

Figure $8 . \quad$ Average monolayers of water by stationary phase................. 28

Figure 9. Schema of MS instrument................................ 33

Figure 10. Triple quadrupole mass analyzer (QqQ) .......................... 40

Figure 11 . Selectivity gained by hyphenation........................... 42

Figure 12. Column chemistries for C18 vs. DH vs. Waters Amide BEH......... 59

Figure 13. Waters Acquity TQD used in this experiment...................... 62

Figure 14. Infusion set-up........................................... 65

Figure 15. QqQ fragmentation schema....................................... 68

Figure 16. Front inlet set-up for this instrument......................... 75

Figure 17. Intellistart recurring error message........................... 76

Figure 18. Intellistart readout highlighting issue with mass accuracy........... 78

Figure 19. Calibration with phosphoric acid in ESI negative mode............... 81

Figure 20. MMA Intellistart readout after MS/MS calibration.................. 82

Figure 21. Intellistart report with only one stable transition................. 83 
Figure 22. Manual tuning and optimization of [M-H] $]^{-}$ion for MMA............. 84

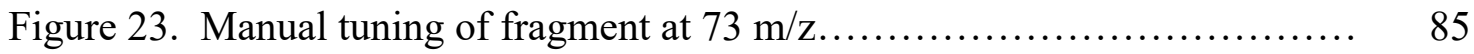

Figure 24. Stable RT on DH -5 injections............................. 89

Figure 25. DH - one injection by transition............................... 90

Figure 26. Classical HILIC/ANP MP mixture on DH. 3 injections plus transitions...

Figure 27. MMA-d3 preliminary injections. (A) DH (B) C18 (C) BEH.......... 94

Figure 28. Three injections in a row without prime....................... 97

Figure 29. Dirty skimmer cone (left). Clean skimmer cone and cone (right)..... 99

Figure 30. ESI capillary probe dislodged............................... 100

Figure 31. C18 repeat injection series................................. 102

Figure 32. DH repeat injection series.................................. 103

Figure 33. Carryover of MMA on C18 column............................. 104

Figure 34. BSM back pressure issue with ethyl acetate prime................. 106

Figure 35. BEH-Amide all 3 analytes................................. 107

Figure $36 . \mathrm{C} 18$ all 3 analytes...................................... 108

Figure 37. DH all 3 analytes............................................ 110

Figure 38. $10 \mathrm{mM}$ ammonium acetate $0.1 \%$ formic acid on $\mathrm{DH}$ by transition..... 114

Figure 39. $10 \mathrm{mM}$ ammonium acetate $0.1 \%$ formic acid on $\mathrm{DH}$ repeats (TIC).... 115

Figure 40. $10 \mathrm{mM}$ ammonium formate $0.1 \%$ formic acid on DH by transition.... 117

Figure 41. $10 \mathrm{mM}$ ammonium formate $0.1 \%$ formic acid on DH repeats (TIC)... 118 


\section{LIST OF ABBREVIATIONS}
ANP - aqueous normal phase
$\mathrm{B} 12$ - vitamin B12
CAP - College of American Pathologists
CoA - coenzyme A
CLSI - Clinical Laboratory Standards Institute
CSF - cerebral spinal fluid
DH - Diamond Hydride
EI - electron impact
FDA - food and drug administration
GC-FID - gas chromatography - flame ionization detector
GC-MS - gas chromatography-mass spectrometry
HETP - height equivalent to a theoretical plate
HILIC - hydrophilic interaction liquid chromatography
HPLC - high-pressure liquid chromatography
HPLC-MS/MS - high-pressure liquid chromatography-mass spectrometry/mass spectrometry
IF - intrinsic factor
LC - liquid chromatography
LC-MS/MS - liquid chromatography-mass spectrometry/mass spectrometry
LDT - laboratory-developed test
MMA - methylmalonic acid
MMA-d3 - deuterated methylmalonic acid
NP-LC - normal-phase - liquid chromatography
PA - Pernicious Anemia
PSI - pounds per square inch
RP-LC - reverse-phase - liquid chromatography, (also known as RPLC)
SA - succinic acid
SA* - 2,2 dimethylsuccinic acid
UHPLC - ultra-high-pressure liquid chromatography
TOF - time of flight
ESI - electrospray ionization
MALDI - matrix-assisted laser desorption ionization
QqQ - triple quadrupole
SIM - selected ion monitoring
TMS - trimethylsilyl trifluoroacetamide
RT - retention time
RRT - relative retention time
MP - mobile phase
TIC - total ion chromatogram 


\section{INTRODUCTION}

\subsection{Background}

\subsubsection{Method Development in the Clinical Laboratory}

The clinical laboratory is designed to take human body fluid samples and measure the concentration of various analytes to assess health or monitor disease progression. The types of body fluids that might be delivered to the clinical laboratory are diverse, including cerebral spinal fluid (CSF), synovial fluid, respiratory secretions, stool, tissue, urine, and blood. Each type of fluid can provide unique information on different disease processes. Urine and blood are the two most common body fluids assayed because of the ease of collection and the wealth of information that can be gleaned from the concentrations of various biomarkers within. Biomarkers are molecules that can be measured and compared to reference ranges to identify pathophysiology or confirm normal physiological function. In summary, the clinical laboratory's goal is quite simple: take fluid from a patient, test that fluid for the presence and concentration of various analytes, and report that information to the clinical staff to inform medical decision making.

The clinical laboratory is a heavily regulated environment as the results produced directly impact patient care and have the potential to produce harm if inaccurate. Clinical laboratories in the United States are overseen by one of two organizations, the Joint Commission or the College of American Pathologists (CAP). While both organizations have different ultimate requirements, they ensure the lab is functioning correctly and are the responsible entities for auditing performance every three years. These organizations 
then issue a laboratory with approval or accreditation, which in essence, is proof that the laboratory is compliant with the critical rules passed in the Clinical Laboratory Improvement Amendments of 1988, as well as other relevant legislation.

In addition to the regulatory standards imposed by these organizations, the Food and Drug Administration (FDA) is responsible for overseeing laboratory diagnostic testing equipment and method design for commercial assays. The process for the FDA to approve an assay is cumbersome and, for some assays, can take years to complete. Often new biomarkers come to light through research that could provide information on a disease process, yet no FDA approved methods exist. In these scenarios, many laboratories with advanced analytical capabilities look to develop an in-house laboratory developed test (LDT) to address the clinical need in a timely fashion.

LDTs are held to a strict set of guidelines and must have acceptable documentation to be considered a fully validated test. The breadth of these experiments is significant and includes criteria such as linearity, carryover, accuracy, and precision, as well as a method comparison. Guidance on the type of experiments required is outlined in several vital documents published by the FDA, CAP, and the Clinical Laboratory Standards Institute (CLSI). One example of specific guidance for LC-MS/MS Method Development is CLSI C62-A. ${ }^{1}$ The rigorous requirements for LDTs are to ensure that patient results are accurate and lead to informed medical decisions. To develop an assay in a clinical laboratory, a thorough understanding of the regulations, biochemistry of the analyte, and instrumentation is required. 


\subsubsection{Methylmalonic Acid}

\subsubsection{MMA Biochemistry}

MMA is an endogenous organic acid that is elevated in two distinct pathophysiological pathways. The presence of an increase in MMA concentration is typically used to diagnose B12 deficiency or the presence of an inborn error of metabolism, a methylmalonic acidemia. ${ }^{2}$ In a healthy individual, MMA concentrations are typically less than $0.40 \mathrm{nmol} / \mathrm{mL}$; however, when an inborn error of metabolism or B12 deficiency is present, the concentration can rise substantially. ${ }^{3}$ The relative absence of MMA in the healthy population and the stark increases with these pathophysiologies make MMA a promising and sensitive biomarker. In order to understand why MMA would increase in these two rather different conditions, which present with quite different symptoms, requires an in-depth look at the biochemistry of MMA. It is worth noting that the increase in MMA concentrations for these two separate scenarios show quite a significant difference in order-of-magnitude, with the increases in methylmalonic acidemias being 100 to 1000 -fold higher than in the case of cobalamin deficiency (B12). ${ }^{2,4,5}$ In addition to concentration differences between the two pathophysiologies, there are differences in presentation, preferred sample type, age of onset, and treatment.

\subsection{Methylmalonic Acidemia}

The presence of a methylmalonic acidemia, an inborn error of amino acid metabolism, is usually diagnosed by a newborn screening test. The preferred sample for this type of screening test is a dried blood spot on filter paper, which is then tested for metabolic disorders, endocrine disorders, hemoglobin disorders, and others. ${ }^{6}$ The 
prevalence of a methylmalonic acidemia is approximately 1 in 30,000 to 1 in 50,000 live births. ${ }^{3,7}$ The detection at an early age for this condition is critical as the reduction of protein consumption can slow the build-up of MMA concentrations in blood. The aberrant pathway in this condition is due to a defect or deficiency in one or several enzymes involved in amino acid catabolism.,

As shown in Figure 1, the breakdown of branched-chain amino acids requires several enzymatic steps with the final delivery of succinyl-CoA to the citric acid cycle. However, fault in any enzymatic step will lead to a build-up of precursors and a lack of progress through this biochemical pathway. The biochemical profile of this pathology includes the build-up of intermediates such as MMA, as well as other metabolites of propionate metabolism including 3-hydroxypropionic, 2- methylcitric acid and tiglycine. ${ }^{4,8}$ 

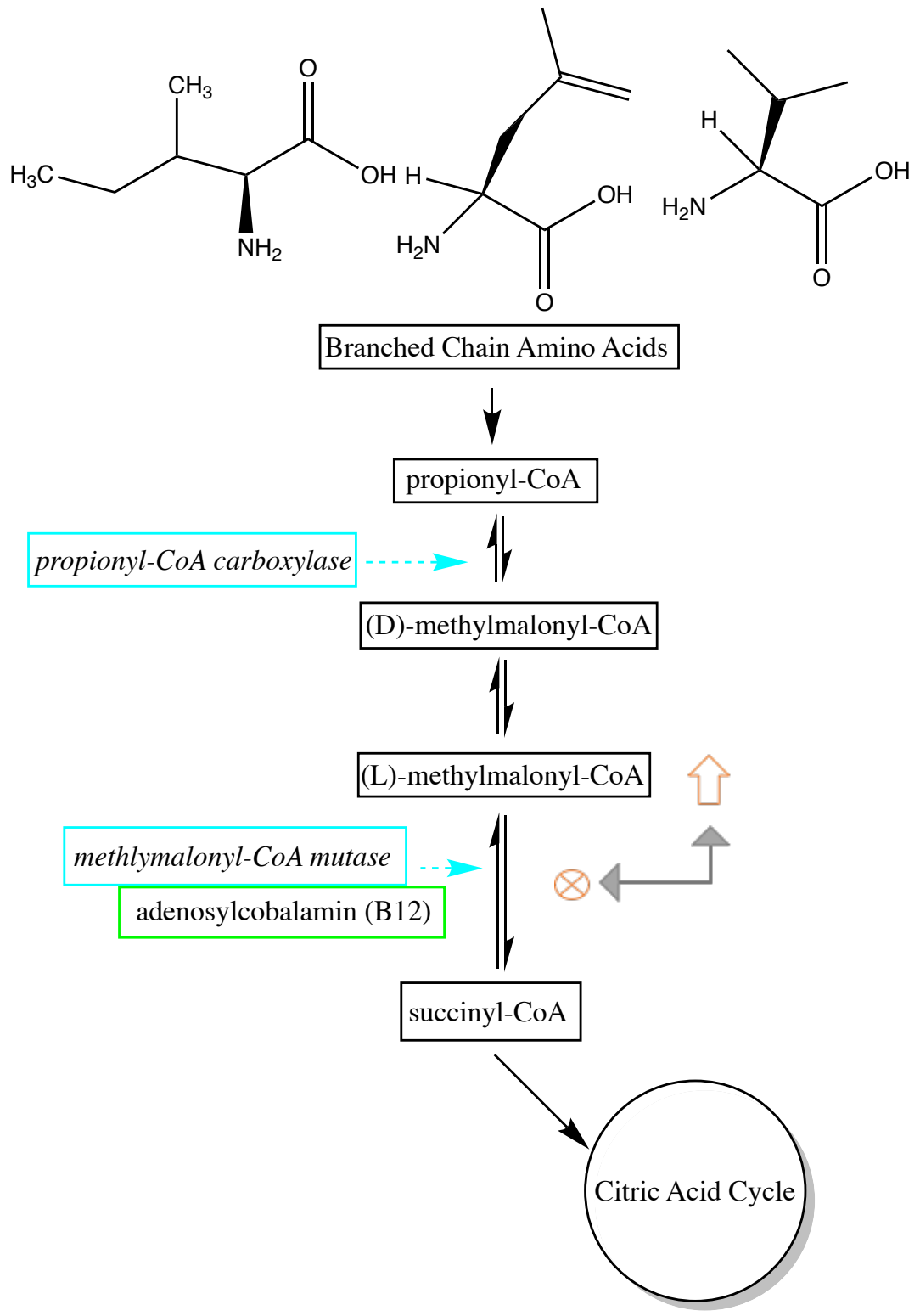

Figure 1. Biochemical pathway of methylmalonic acid. The amino acid catabolism shown has intermediates displayed in black, enzymes displayed in blue boxes, and the cofactor B12 is shown in a green box. The orange $\mathrm{x}$ and arrow illustrate the elevation of (L)-methylmalonyl-CoA when this step in the pathway is inhibited.

Depending on which step in the pathway has the enzymatic deficiency, patients with this inherited condition may be unable to convert propionyl-CoA to methylmalonyl-CoA 
or methylmalonyl-CoA to succinyl-CoA. One consequence of these enzymatic deficiencies is the prevention of branch chain amino acids' end products from entering the Krebs cycle. Although not all are displayed above, the diverse list of mutations that are possible include, mutations in methylmalonyl-CoA mutase, methylmalonyl-CoA epimerase and cobalamin A, B, C, D, F and J deficiencies. ${ }^{9}$ The block in the final step before the citric acid cycle, the conversion of (L)-methylmalonyl-CoA to succinyl-CoA as indicated by the "X" in Figure 1 results in the most substantial elevations of MMA concentration in blood. The presentation and severity will vary depending on specific mutation or combinations of several, but many of these deficiencies can be life-threatening.

The acute presentation in newborns, due to the massive increases in MMA, up to 10,000 times the normal reference range results in a severe pathology, often entailing metabolic acidosis, hyperammonemia, and rapid deterioration. ${ }^{4,10}$ Failure to thrive is the blanket clinical diagnosis given to infants before laboratory findings are available, indicating the severity of the condition and its life-threatening nature. Treatment for metabolic disorders is mainly based on avoidance of the foods that feed into defective pathways. In the case of branched-chain amino acids, avoidance of whey, beef, chicken, fish, and whole wheat, amongst other specific foods that have elevated BCAAs, would be recommended. ${ }^{11}$ While B12 deficiency may be present in the newborn population, the presentation is more subtle, and MMA concentrations are only mildly elevated. 


\subsection{B12 Deficiency}

The second pathway that results in a mildly elevated MMA concentration is B12 deficiency, which is often suspected in an older population due to a hematologic malignancy on a blood smear review. ${ }^{12}$ Megaloblastic anemia is the finding that suggests an investigation of B12 status. Cobalamin, B12, is a cofactor for several enzymatic pathways in humans, including red blood cell formation and DNA production. ${ }^{13} \mathrm{~A}$ cofactor is needed to bind certain enzymes to make them functional. As a cofactor, B12 in the form of adenosylcobalamin is needed to complex with the enzyme methylmalonyl- coenzyme A (CoA) mutase for normal function. In the case of B12 deficiency, the patient may not have any enzymatic deficiencies in the biochemical pathway shown in Figure 1. However, without the required B12 as a cofactor, methylmalonyl-CoA mutase will underperform. Methylmalonyl-CoA mutase is responsible for the final step in the conversion of L-methylmalonyl-CoA to succinylCoA. ${ }^{14}$ The increase in MMA concentration in B12 deficiency is mild relative to what is observed in the case of enzymatic defect.

While B12 is a cofactor for methylmalonyl-CoA mutase, a fact which is used to tie a rise in MMA concentration to a deficit of B12, it is also a cofactor in other enzymatic pathways. B12 is also a cofactor for methionine synthase that is responsible for the conversion of homocysteine to methionine, which is required for red blood cell formation (Figure 2). 

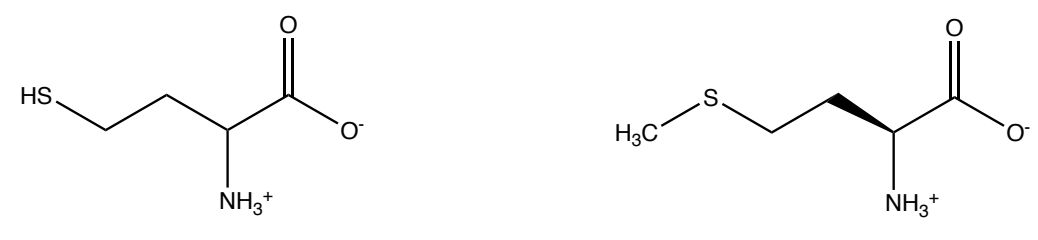

homocysteine

methionine

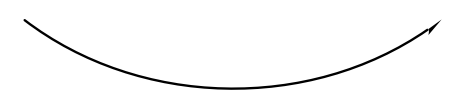

B12 Required as Cofactor
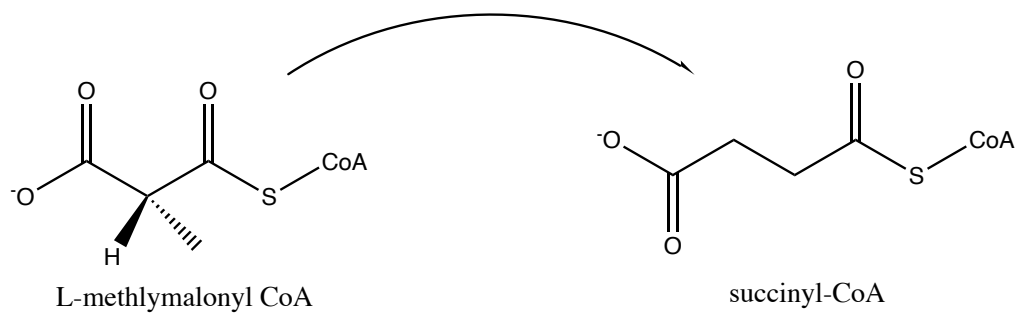

Figure 2. Two important B12 dependent pathways. B12 is an essential cofactor in DNA synthesis (with methionine synthase) and branched-chain amino acid catabolism (with methylmalonyl-CoA-mutase).

While the decrease in methionine synthase's efficacy does result in an increase of homocysteine, other conditions cause this increase, making homocysteine less specific of a biomarker than MMA for B12 deficiency. The lack of adequate cofactor for these enzymatic steps can have variable presentation depending on the severity of the B12 deficiency. However, the typical presentation is macrocytic anemia with neurological deficits. Other conditions can cause macrocytic anemias and neurological deficits, so the ability to test MMA provides a more in-depth understanding of the cause of the pathology.

While it may seem logical that B12 would be the preferred test for B12 deficiency, this is surprisingly not the case. B12 is mainly stored in tissues, and tissue stores can become depleted long before the serum value is lower than the normal reference range. ${ }^{5,15,16}$ Interestingly, while B12 is a simpler molecule to test in some regards, it 
provides less information biochemically than MMA. Therefore, MMA is commonly measured as a functional biomarker of B12 status, although no FDA approved assay for MMA is available commercially. ${ }^{10}$ Analytical testing of MMA as a biochemical marker of B12 status is typically performed on a plasma sample; whole blood is centrifuged to separate the liquid from the cellular elements. A typical reference range quoted in the literature for MMA elevation due to B12 deficiency is $60-360 \mathrm{nM}$, but this can vary based on the laboratory performing the testing and the testing methodology. ${ }^{3,5}$ While B12 deficiency is not necessarily life-threatening, like the inherited inborn errors of metabolism discussed previously. Early detection is still essential as it allows for early treatment and often complete reversal of symptoms. Symptoms of B12 deficiency include muscle weakness, anemia and neurological deficits. ${ }^{12}$

Treatment for B12 deficiency involves the administration of B12, either through changes in diet, supplementation, or injection. The fact that many foods have B12 makes treatment with diet a plausible response assuming that the patient does not suffer from pernicious anemia (PA), which is a condition that prevents B12 absorption in the gut due to issues with intrinsic factor (IF). ${ }^{17}$ If the patient does suffer from lack of access to foods containing B12 or has issues with absorption, there are injectable options that circumvent these issues. Unlike MMA elevation due to inborn errors of metabolism, MMA increases due to B12 deficiency are responsive to B12 supplementation. The enzymes are normal, but the lack of cofactor results in the MMA build-up. Despite the apparent clinical need for an assay that enables early detection of a condition that is treatable, B12 deficiency, few clinical laboratories can quantitate MMA in house. One 
may wonder if MMA is such a useful biomarker, why is there no FDA approved commercial assay for this analyte?

Before elucidating some of the challenges in assay design that result from the structure of MMA, it should be made clear that testing for B12 directly has a host of limitations. Therefore, despite the analytical challenges of MMA measurement as a surrogate for B12 status, MMA is still the preferred biochemical marker to diagnose this condition. The low concentration of MMA in the healthy population combined with the stark increases when B12 is deficient make MMA the most sensitive assay currently available as a functional biomarker of B12 status at the tissue level. ${ }^{18}$ There are two predominant issues with testing B12 directly, one is a physiological issue, and the other is an analytic issue. Testing serum B12 to approximate tissue stores in patients can often be misleading, where values in the normal to low normal range for B12 are measured, yet the patient begins to develop symptoms of B12 deficit. Analytically, the issue is the lack of standardized reference materials and the wide variety of platforms and methods by which B12 is currently tested. ${ }^{19}$ The variety of testing methods for B12 include IF-based automated competitive assays, microbiological assays using Lactobacillus leichmanii and radioimmunoassay. The diversity of these assays, each with its own set of interferants and limitations, confound agreement across platforms. Testing MMA has its own challenges, mostly due to the structure, size, and polar nature of the molecule. 


\subsubsection{MMA Structure}

The clinical utility of an FDA approved assay for MMA is clear. However, there are challenges in developing this assay due to the structure of MMA and the presence of an endogenous interferant succinic acid (SA).
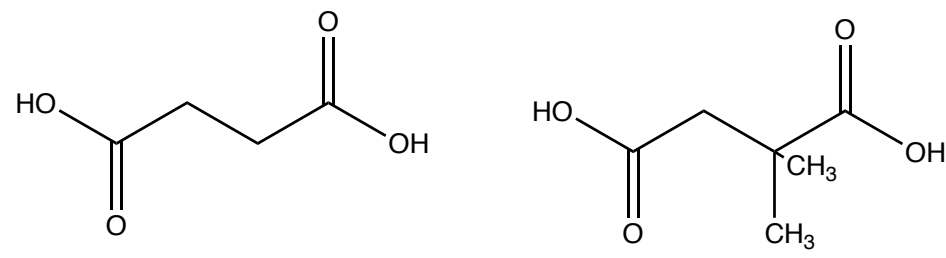

$\mathrm{SA}=118.09 \mathrm{~g} / \mathrm{mol}$

$\mathrm{SA}^{*}-146.09 \mathrm{~g} / \mathrm{mol}$<smiles>CC(C(=O)O)C(=O)O</smiles><smiles>O=C(O)C(C(=O)O)C(=O)O</smiles>

MMA $=118.09 \mathrm{~g} / \mathrm{mol}$

MMA-d3 - $121.11 \mathrm{~g} / \mathrm{mol}$

Figure 3. Structures of analytes. All analytes relevant to this study are displayed. SA is succinic acid. SA* is 2,2-dimethylsuccinic acid. MMA is methylmalonic acid. MMA-d3 is the deuterated internal standard. Structures shown prevail under acidic conditions.

Structurally, MMA is a dicarboxylic acid (Figure 3). It is a small polar molecule with a low physiologic concentration, and its structural isomer SA is in higher concentration physiologically. ${ }^{5}$ The small size, polar nature, and presence of a high concentration endogenous interferant present challenges for several testing methodologies. The pKa's for the two carboxylic acid groups for MMA are 3.07, and 5.76. ${ }^{20}$ Ideally SA would be employed in method development for MMA to ensure the two endogenous isomers can be chromatographically separated; however 2,2 dimethylsuccinic acid (SA*) was used in 
place of SA due to availability at Valley Medical Center laboratory. The retention time of SA cannot expect to be mirrored by SA*. However, chromatographic trends and investigation of this similar compound's behavior can be used to inform the chromatographic control of SA. MMA has been tested by several methods by various research groups; subsequently the most common methods will be compared, and the limitations will be discussed.

\subsubsection{Testing Strategies for MMA}

The variety of testing methods for MMA in the literature includes different chromatography varieties with or without mass spectrometry and capillary electrophoresis. MMA has been quantitated with gas chromatography combined with a flame ionization detector (GC-FID), gas chromatography-mass spectrometry (GC-MS), high-performance liquid chromatography (HPLC) and LC-MS/MS. Another technique that will be discussed is immunoassay, as this is, by far, the most prominent analytical technique used in the clinical laboratory. It will be shown in subsequent sections that although all of these techniques are used in the clinical laboratory for various analytes, the most promising method for the quantitation of MMA is LC-MS/MS, particularly with a chromatographic approach that favors retention of polar compounds. To explain the literature methods in appropriate detail, a discussion of the analytical techniques commonly used is warranted.

\subsubsection{Brief Review of Analytical Methods in the Clinical Laboratory}

The modern clinical laboratory is dominated mainly by immunoassay and spectrophotometric techniques based on Beer's Law to quantitate analyte concentrations 
monitored by absorbance (Figure 4, 5). These techniques can be used in tandem or stand-alone fashion, such as lateral flow immunoassay or coupled chemical reactions with a photometric endpoint.

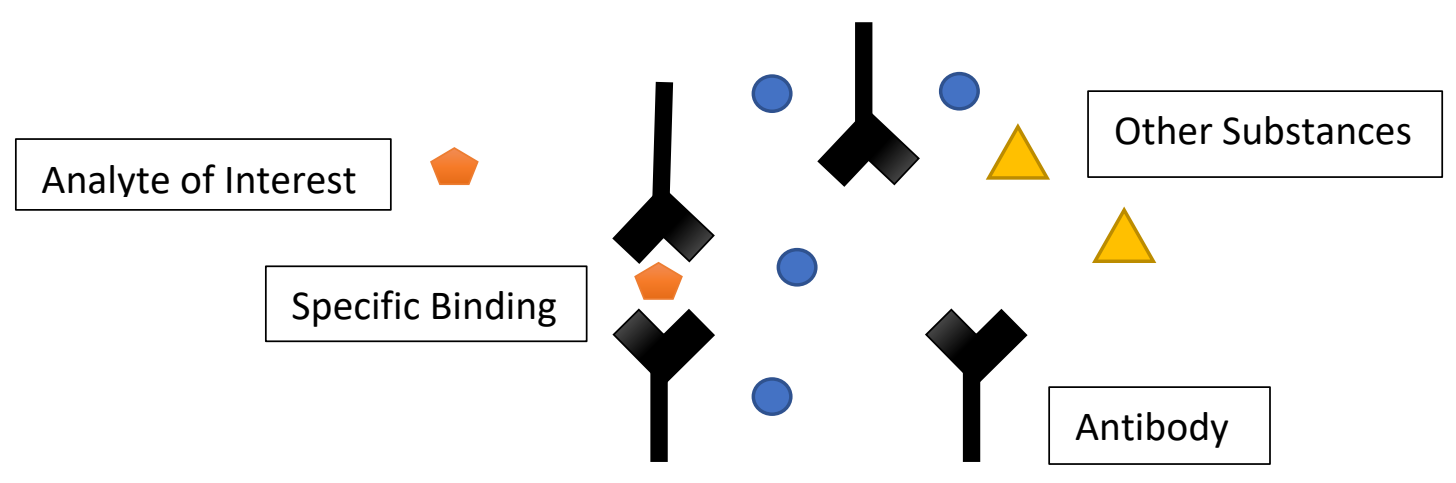

Figure 4. Immunoassay. A simple schematic displaying the specific interaction of analyte and antibody.

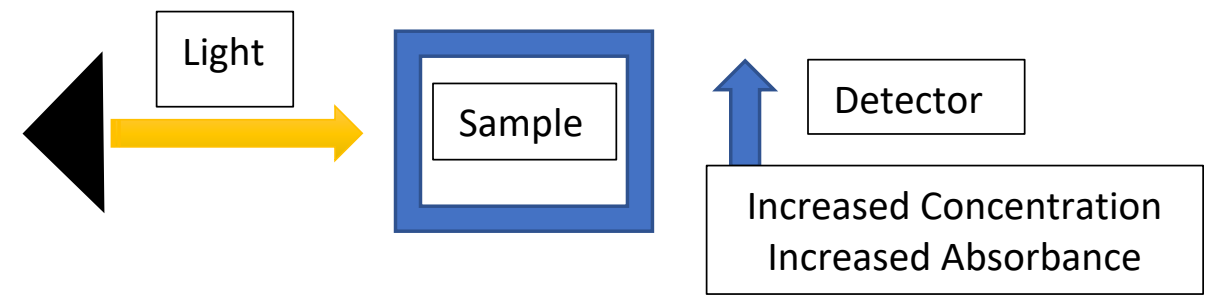

Figure 5. Beer's Law. A schematic showing the principle and set-up of an instrument that uses absorbance to calculate concentration.

$$
\text { Eq. 1. } A=\boldsymbol{\epsilon} * \boldsymbol{l} * \boldsymbol{c} \longrightarrow A \propto \boldsymbol{c}
$$

In a spectrophotometric measurement, incident light is directed at a sample, which will allow transmission of a fraction of that incident light inversely proportional to the concentration of the analyte in the sample. In simple terms, Beer's Law boils down to absorbance (A) is proportional to the concentration(c) (Figure 5). It is possible to increase specificity by coupling chemical reactions with a chromogenic endpoint, or 
labeled antibodies can bind small molecules in solution to increase absorbance. Despite these attempts to make spectrophotometry more specific, there is always the possibility of an unknown pigment in the sample matrix or unseen biomolecules that absorb at the same wavelength other than the analyte of interest. Therefore, while Beer's Law underpins many measurements in the clinical laboratory, there are several inherent flaws that chromatography, coupled with mass spectrometry, can mitigate.

Immunoassay is an analytical technique in which a reagent antibody is used to preferentially interact and bind an analyte of interest by recognizing an epitope on that analyte (Figure 4). Yalow and Berson invented immunoassay in 1960, which boasted the specificity of this antibody-epitope interaction and was commercially available for a wide variety of biomolecules. ${ }^{21}$ The diversity of applications and widespread commercialization, including automated testing lines and the perceived specificity, have allowed immunoassay to be the predominant testing method in the clinical lab for the last sixty years. Despite the continued antibody engineering and the significant investments in this technology over the years, there are several issues inherent to the technology that persist today. Immunoassay suffers from specificity issues due to cross-reactivity, autoantibody interference, hook effect, heterophile antibodies, and standardization issues due to the proprietary nature of reagent antibody development.

Even with immunoassays known issues with specificity compared to LC-MS/MS, it would take a federal mandate to challenge this status quo and introduce a more powerful technique into the clinical laboratory. In 1981 a US airplane crashed into the Nimitz aircraft carrier, prompting President Reagan to adopt an aggressive drug screening policy. 
Immunoassay, which was the predominant testing method at the time for drugs of abuse, had a very high false-positive rate, making the testing unreliable. The flaw of crossreactivity for immunoassay of small molecules was exposed, which prompted a 1988 federal mandate that stated all drug of abuse testing must be performed by a confirmatory method such as GC-MS. ${ }^{22}$ Since this initial foothold in the late 1980s, applications for chromatography and mass spectrometry in the clinical laboratory have steadily grown. Although the sensitivity and specificity of the hyphenated techniques of GC-MS and LC-MS/MS are quite impressive, they still only hold niche roles in the clinical laboratory and have not surpassed immunoassay and spectrophotometry, the dominant techniques.

In order to simply demonstrate the power of combining a separation technique such as chromatography, with an incredibly specific measurement technique like mass spectrometry, consider the typical clinical sample. A clinical sample can be blood, urine, or any other body fluid used to measure a specific analyte or biomolecule of interest. The sample will have a matrix, which is the fluid portion of the sample, including all other constituents of interest other than the analyte. Interfering substances can include any number of proteins, lipids, or biomolecules that may confound or interfere with an accurate measurement. Each person can have a wide variation in the amounts of each of these substances. Imagine a sample is taken just as it is, matrix and analyte, no separation performed before generating a mass spectra. That mass-spectra would be very difficult to deconvolute as the analyte of interest would essentially be a needle in the haystack. 
The benefit of adding a separation modality such as chromatography allows the delivery of different compounds to the detector in discrete packets and drastically increases the signal-to-noise ratio. As chromatography and mass spectrometry are both stand-alone techniques with rich histories, they will be discussed separately to explain the underlying theory and instrumentation. Once they are understood in isolation, combining them as a powerful analytical measurement technique will be discussed.

\subsubsection{Chromatography}

\subsubsection{History of Chromatography}

Chromatography has become a powerful analytical technique with many applications that allows the separation of a complex sample into its various constituents by the differential interaction of the analytes with both the mobile and stationary phases. Currently, the term chromatography refers to a whole arm of separation science. However, it began as an observation regarding the migration of plant pigments by a Russian botanist, M.S. Tswett, at the turn of the $20^{\text {th }}$ century. The credit for beginning the field that eventually became chromatography is given to Tswett, for his observation of the differential migration of chlorophyll relative to carotenoids in a packed calcium carbonate column with petroleum ether as the mobile phase (MP). ${ }^{23}$ Since these humble beginnings, the utility of a customizable chemical separation that can deliver components of a complex mixture to a detector for accurate quantitation has revolutionized analytical chemistry. Select chromatography applications as they apply to the field of analysis in the clinical laboratory will be discussed in subsequent sections. 


\subsubsection{Important Concepts in Chromatography}

Over the last 100 years, the field of chromatography has blossomed into a scientific discipline unto itself, with its own language and doctrines, which will be examined in a limited fashion in this section. A chromatographic experiment is one in which a sample, often a complex mixture, is mixed with a solvent and passed through a stationary phase, often a column. This complex mixture will dissociate in the solvent, also known as a MP. While passing through the packed column, various molecules in the mixture will have fleeting chemical interactions with the stationary phase particles. There are various chemical interactions possible with the surface chemistry of these stationary phase particles, including ionic interactions, van der Waals interactions, hydrogen bonding, adsorption, and even partitioning into localized solvent layer particle-mobile phase interface. Depending on the degree of interaction between the particles and the analytes, the various compounds will be retained for different lengths of time before eventual elution in the MP.

The retention time concept is a critical one in chromatography, which means time retained within the column. Every chromatographic instrument will develop an output graph, known as a chromatograph, which will list retention time on the $\mathrm{x}$-axis and some form of detector counts on the y-axis. In a well-designed chromatographic experiment, the compounds being measured will elute independently at their defined retention times, and they will also elute reproducibly. In other words, retention time can be a defining characteristic for an analyte, especially if internal standards are used to calculate a relative retention time. An internal standard is a molecule that should ideally be nearly 
identical to the analyte. Isotopically labeled internal standards are popular for use when coupled with a mass spectrometry-based detection method. The use of an internal standard that is chemically similar to the analyte of interest allows the scientist to adjust for small amounts of chromatographic drift using the relative retention time calculation. Calculating a relative retention time (RRT) will allow the retention time (RT) to remain a defining characteristic even in situations where the column length changes (typically in $\mathrm{GC}$ ), the flow rate changes, or other parameters affecting absolute retention time are altered.

$$
\text { Eq. 2. } R R T=\frac{R T_{\text {Analyte }}}{R T_{\text {Internal } \text { Standard }}}
$$

The key parameters affecting chromatographic quality, elution order, and peak shape can fill a whole chapter of text. However, several key definitions are critical in understanding how to manipulate a separation. In simple terms, the goal of a chromatographic experiment is to achieve optimal separation in the minimum time required. Resolution, capacity factor, selectivity, and efficiency will be discussed as necessary concepts to describe a chromatographic experiment. Resolution can be thought of as the ability to see two things as distinct. For example, if two small circles were drawn on paper and that paper was moved farther and farther from the viewer, initially, the two circles would be well resolved. However, as the distance increased, the circles may blend into one from the observer's viewpoint. In this example, the resolving power of the eye is limited to a certain distance. In chromatography, resolution (R) is defined as the difference in the retention time of the two peaks eluting from the column, divided by the peak width. However, resolution (R) is dependent upon several variables, 
including capacity factor $(k)$, selectivity $(\alpha)$, and efficiency $(\mathrm{N})$, as described by the Purnell Equation (Eq. 3).

$$
\text { Eq. 3. } R_{S}=\left(\frac{\sqrt{N}}{4}\right)\left(\frac{k}{k+1}\right)\left(\frac{\alpha-1}{\alpha}\right)
$$

To achieve excellent resolution, one must control each of the variables described in the Purnell Equation. The three terms in the Purnell Resolution Equation can be related to efficiency, retention, and selectivity, respectively. A suitable resolution is typically considered to be "baseline," or in other words, the tails of the peak do not overlap but has also been numerically described as a number greater than 1 to $1.5 .^{24}$ The capacity factor (k), is used to measure the degree of retention of a solute relative to an unretained solute (Eq. 4). The selectivity of a column for two peaks is defined as the ratio of the two peaks' capacity factor, with a higher number denoting greater selectivity and, in theory, enhanced ability to separate (Eq. 5). However, the peaks can be too wide to resolve without decent efficiency despite the selectivity or capacity factor. Efficiency is a concept that is described by the Van Deemter Equation.

$$
\begin{gathered}
\text { Eq. 4. } k=\left(t_{r}-t_{0}\right) / t_{0} \\
\text { Eq. 5. } \alpha=\frac{k_{2}}{k_{1}}
\end{gathered}
$$

\subsubsection{Van Deemter Equation}

The Van Deemter Equation (Eq. 6) is a mathematical expression that directly addresses peak shape and width, critical variables for defining the efficiency parameter in the Purnell Equation (Eq. 3). The ideal peak is sharp, with no skew, allowing for the integration of the area underneath and subsequent accurate quantitation. Due to the 
principles of diffusion and mass transfer, there is a characteristic Gaussian distribution for ideal chromatographic peaks.

\section{Eq. 6. $H E T P=A+\frac{B}{\mu}+C * \mu$}

The Van Deemter equation is composed of three parts, the A term, the B term, and the $\mathrm{C}$ term. The $\mathrm{A}$ term is independent of flow rate, but the $\mathrm{B}$ and $\mathrm{C}$ terms are changed based on the MP flow rate $(\mu)$. The A term is to account for what is known as eddy diffusion, which describes the reality that in a column packed with small particles, there are many paths that an analyte can take to reach the detector. Since the analyte has this ability to take divergent paths, it would make sense that this compound will arrive at the detector at slightly different times, resulting in a broader peak. A column packed with smaller, more uniform particles will decrease this term and result in a sharper peak, but this will increase back pressure to the fluidics system.

The second term, B term, describes longitudinal diffusion, which is the process by which an analyte diffuses forwards and backward from the region of high concentration to a lower concentration. The B term is inversely related to MP linear velocity, as a faster flow rate will limit the amount of time the analyte has to diffuse longitudinally. Looking at the B term alone, it would seem the faster the flow rate, the sharper the peak, but the $\mathrm{C}$ term will show an unfavorable relationship with increased flow rate.

The last term in the Van Deemter equation, the $\mathrm{C}$ term, describes resistance to mass transfer. The $\mathrm{C}$ term is increased proportionally to the linear velocity of the MP. Mass transfer can be thought of the attraction and movement of as the analyte onto the stationary phase and then back into the MP. As the flow rate increases, the analyte and 
MP will travel past the stationary phase at an increasing speed, thereby decreasing the amount of time possible for interaction and mass transfer. When all three of these terms are taken together, a parabolic function dependent on flow rate can be calculated that can be used to determine the ideal linear velocity for the MP to achieve the highest efficiency, also known as height equivalent to a theoretical plate (HETP). It should be noted that efficiency is primarily defined by the flow rate and column dimensions, such as particle size and uniformity. In comparison, the selectivity and capacity factors are decided mainly by the functional groups bonded to the stationary phase particles and the chemical interaction of these functional groups with the analytes in the sample. In other words, it is possible to get variations in elution order, by changing column surface chemistry, while holding constant flow rate and particle size. These chemical principles defined above can be applied in several different separation modes, a select few of which will be discussed in subsequent sections.

\subsubsection{Modes of Separation}

While the most of this paper discusses concepts involved in LC, there is some description of GC in subsequent sections that necessitates a basic understanding of the concepts defined herein. In general, chromatographic experiments can be either isocratic or gradient elution profiles. In gas chromatography, the variable being modified during a chromatographic run is typically temperature, with no change in the column oven temperature during a run referred to as isothermal. In LC isocratic or gradient refers to MP composition, with isocratic describing a constant MP composition and gradient representing a change in the percentage of the weak and strong MPs throughout the 
course of the experiment. The chromatographic modes described in the following sections will vary the column chemistries and MP compositions of the weak and strong MPs, resulting in preferential retention of various analytes.

\subsection{Reverse Phase vs. Normal Phase}

Reverse-phase liquid chromatography (RP-LC) has been the clinical laboratory's workhorse since the entrance of LC-MS/MS in this environment in the 1990s. The typical RP-LC experiment consists of a non-polar column chemistry such as C18, with an aqueous or weak MP and an organic solvent as the elution or strong MP. Typical aqueous MPs include water, often with the presence of buffer salts and formic acid in low concentrations for compatibility with detection by mass spectrometry. In an RP-LC experiment, typical strong MPs are acetonitrile and methanol, often with the same buffer salts and formic acid as the aqueous MP.

The overarching concept in chromatography is that like stays with like due to the fleeting chemical interactions described earlier. In general, this is simplified to elution based on polarity, if the column is non-polar, the greasy alkyl chains will interact most substantially with non-polar compounds and thus will retain those compounds with the greatest strength. Suppose one considers the relative affinity for a stationary phase as compared to the MP. In that case, it may become evident that an aqueous MP would not have the ability to interact with a non-polar molecule with greater strength than the nonpolar stationary phase. However, as the MP composition changes throughout the gradient, the increased organic content of this MP can interact favorably with the analyte and cause the analyte to elute. In short, an RP-LC experiment is ideal for non-polar 
compounds. Analytes will elute in the order of decreasing polarity, with the most polar compounds eluting early and the least polar eluting last.

Normal phase can be thought of as the opposite of RP-LC. In a typical NP-LC experiment, a polar column is used to retain polar compounds. In this case, an organic solvent is used as the weak MP, and a slightly more polar organic solvent is used as the strong MP. In NP-LC, an example of a weak solvent is hexane, and a strong MP is isopropanol or ethyl acetate. The elution order for compounds in NP-LC is opposite of RP-LC, the least polar elutes first, and the most polar is retained the longest, on the column. NP-LC is not a very common technique in the clinical laboratory. In contrast, RP-LC is the predominant technique with the most diverse column chemistries and the number of application notes made by manufacturers of HPLC-MS/MS instrumentation. While RP-LC may dominate the number of current clinical applications, it is obvious it has limitations to its scope of applications for polar molecules.

Despite the dominance of RP-LC, the reality is that many biological molecules, analytes in clinical assays, are polar. The polar nature of drug metabolites and many other biological compounds is a result of how the liver and kidneys process molecules to ensure they can be excreted from the human body. The liver has a two-phase metabolism that substantially increases the molecule's polarity so that the kidneys can excrete the molecule in the urine. This hepatic-renal clearance mechanism does not only affect the composition of urine, but as urine is simply an ultrafiltrate of blood, it affects the metabolite composition in blood as well. Despite this physiological reality that much of that which is sought to be measured is polar, RP-LC has remained the predominant 
technique. It has been said by Abraham Maslow in his book, the Psychology of Science, "if all you have is a hammer, everything begins to resemble a nail."." In the context of chromatography, the hammer has been RP-LC, but in the last couple of decades, a shift in understanding has opened up the idea that perhaps a hammer is not what is needed. That there are better ways to address these physiological challenges than lengthy sample preparatory schemes to make biomolecules less polar before analysis. Two additional separation modes have emerged to address the challenge of chromatographic separation of polar molecules, HILIC, and ANP.

\subsection{HILIC vs. ANP}

In order to address the lack of representation for small-polar analytes in broad, untargeted assays and utilize a more substantial portion of the theoretical separation space available it became clear that divergence from RP-LC was necessary. ${ }^{26-29}$ Beginning in the early 1990s, two groups independently worked on solutions to this problem, the goal being a new stationary phase and chromatographic mode that could operate orthogonally and complementarily to RP-LC. Alpert is credited with performing the early work on HILIC in 1990 which hinged on retention by a partition mechanism (Figure 6) that was a result of a hydrophilic stationary phase with a diffuse adsorbed water layer. ${ }^{27,30}$ 


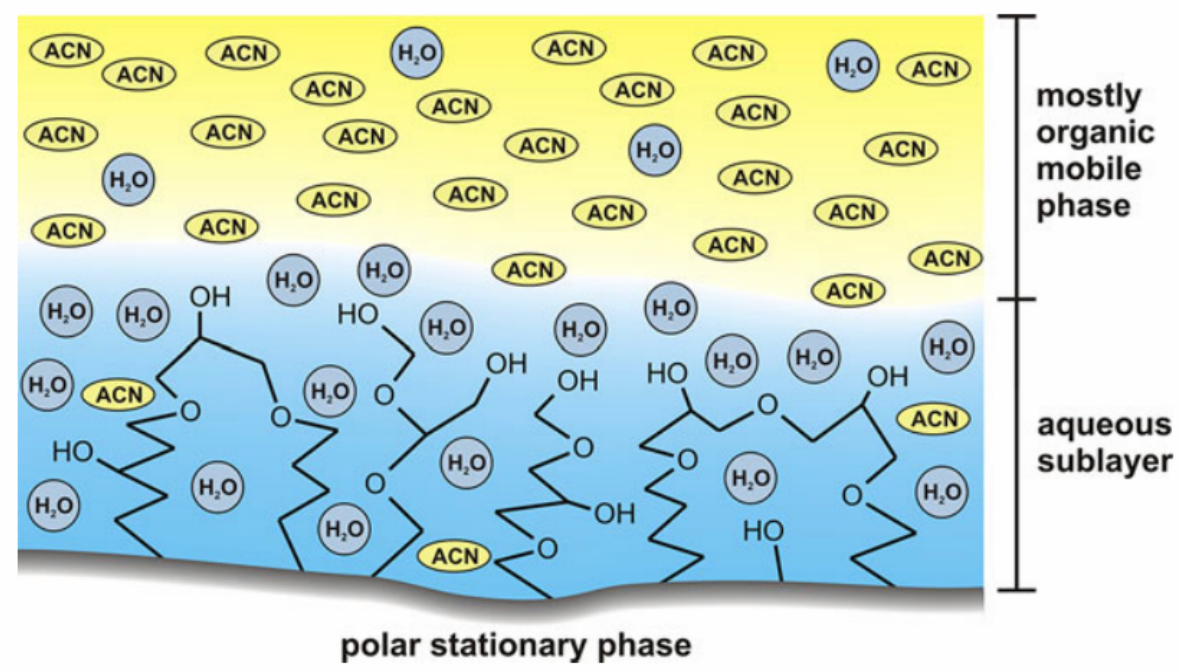

Figure 6. Partition mechanism in HILIC. An image representing the water layer that develops on a stationary phase that has silanols present. HILIC stationary phases operate by a partition mechanism. Reproduced with permission. ${ }^{31}$

The MP composition in a HILIC method is an organic MP that is miscible with water as the weak solvent, such as acetonitrile or methanol, with water as the strong elution solvent. The material used as chromatographic support in a HILIC experiment is a TypeB silica bead, which has -Si-OH groups on the surface that encourage the formation of a water layer. ${ }^{29,32}$ Although HILIC is described as a multimodal retention mechanism, a deep water layer with hydrogen bonding suggests partition is the primary retention mechanism. Another crucial distinction of HILIC chromatography is that the increase in salt concentration increases retention. For example, the use of high concentrations of ammonium acetate of ammonium formate in the MP will cause later acidic analyte elution, relative to MPs with lower salt concentrations. ${ }^{33}$ The consensus for HILIC is that there are several flaws, such as low reproducibility, large volumes of MP required to equilibrate the column, and resultant negative impacts on chromatographic run time, 
which prevent adoption as a widely used clinical tool. However, despite some of these concerns, several groups have shown HILIC to be a promising method to separate polar molecules that can be paired with MS/MS for accurate quantitation. ${ }^{26}$ In summary, Type B silica particles used in HILIC, when functionalized with polar moieties can effectively separate and retain polar molecules and do so by a partition mechanism. While the ability to separate polar analytes is a feature shared by ANP and HILIC, the mechanism and some of the drawbacks are not shared. While both modes can perform a similar function, they do so in quite a different manner. The significant difference in the retention mechanism between ANP and HILIC lies in the hydrophilicity of the silica bead used (Figure 7).
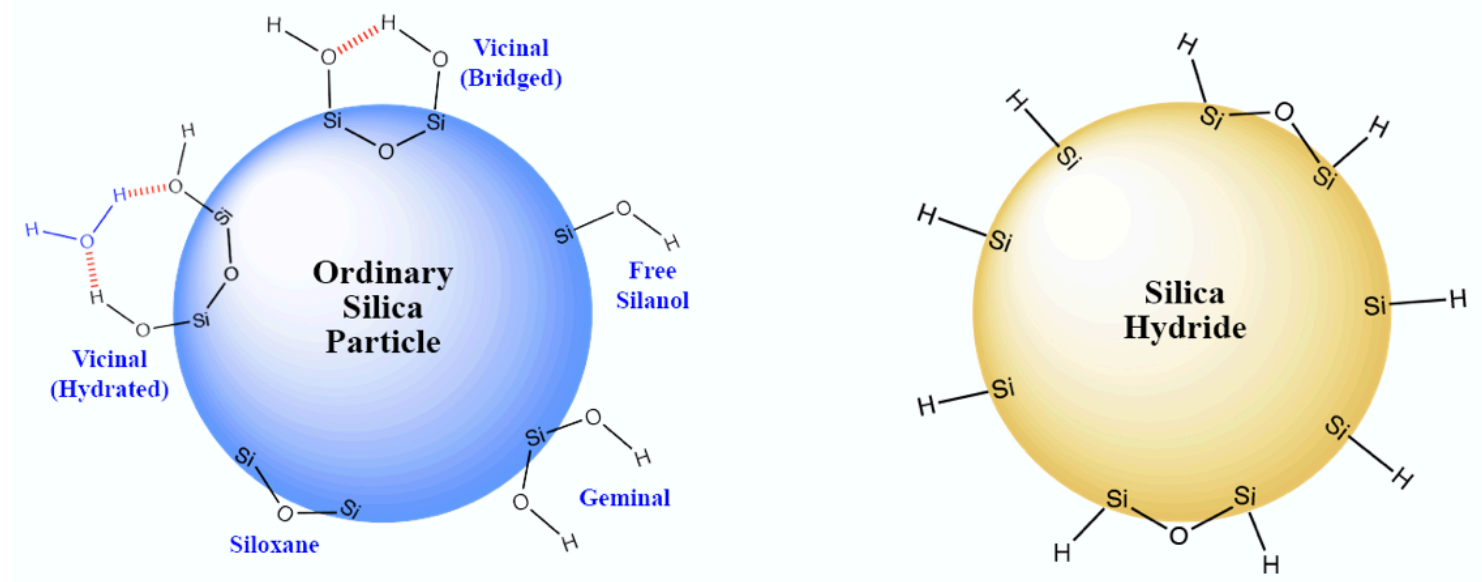

Figure 7. Type B vs. type $C$ silica. On the left, a type B silica particle, on the right, the type $\mathrm{C}$ silica particle is displayed. Note the silanols on type $\mathrm{B}$ versus the silica hydride moieties on type $\mathrm{C}$ silica. Reproduced with permission. ${ }^{34}$

ANP-LC development began around the same time as HILIC, in the early 1990s, with preliminary work published by the Pesek group. ${ }^{32}$ The physical difference between these two polar separation modalities lies in the type of silica used in the column stationary 
phase, type B - HILIC, vs. type C - ANP. The significant difference between these two types of silica is the presence of various silanols in type $B$, with type $C$ having silica hydride functional groups instead of silanols (Figure 7), which has many advantages, including influencing the retention mechanism. The predominant retention mechanism in an ANP-LC experiment is not partition, but rather an adsorption mechanism. The retention mechanism for ANP is still being investigated, and only in the last ten years were significant leaps in the understanding made. This increase in understanding has allowed scientists to clearly distinguish ANP from HILIC, something that the prior 20 years of research failed to do, often lumping the two techniques together.

A leap in understanding regarding the differences of these two techniques came about after Soukup et al. published a paper investigating the number of monolayers of water present on the stationary phases of various columns geared towards polar analytes. ${ }^{29,31,35}$ The work of Soukup et al. is in agreement with the message professed by the Pesek group. Although, HILIC and ANP may show utility for a similar class of molecules; they do so by very different retention mechanisms. HILIC stationary phases were shown to have multiple monolayers of water present, for some columns as many as nine layers were measured. Whereas, ANP stationary phases had less than a monolayer of water present, proving that a partition mechanism would not be possible. ${ }^{32,35}$ Figure 8 below shows the average number of monolayers of water on a variety of stationary phases, in an attempt to demonstrate the difference in ANP vs. HILIC retention. ${ }^{31}$ 


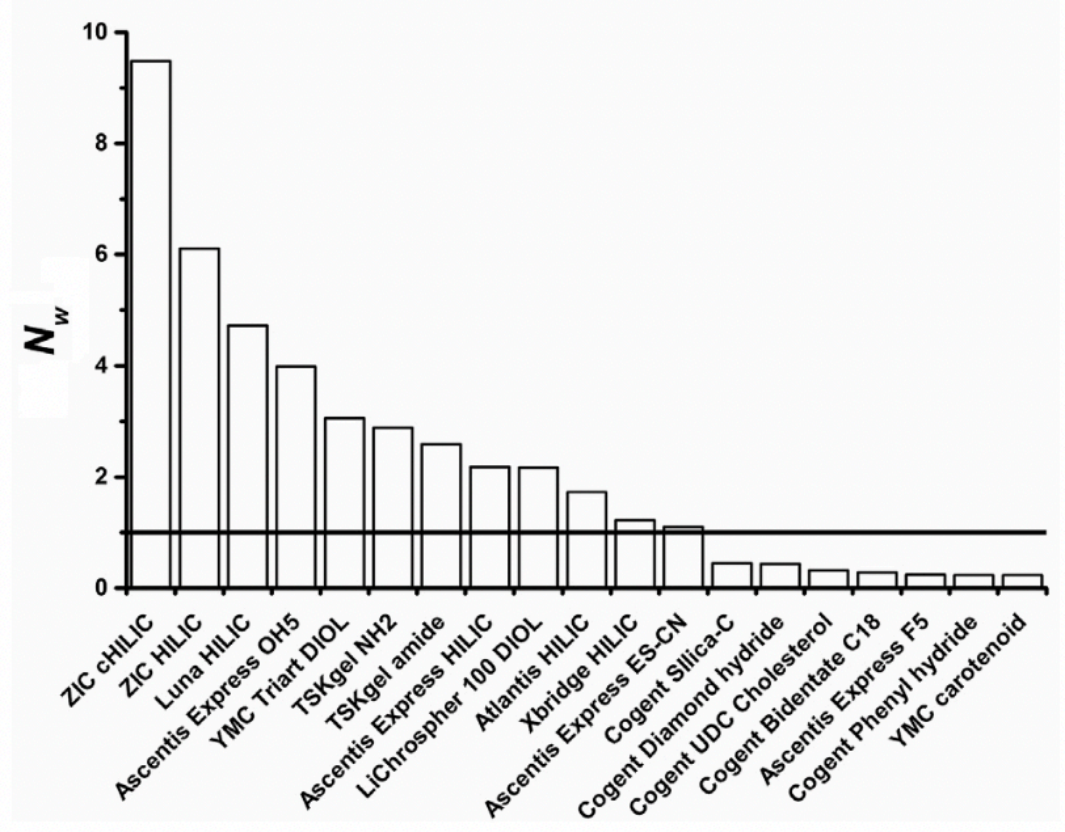

Figure 8. Average monolayers of water by stationary phase. Columns with multiple monolayers of water present favor retention by partition and can be defined as HILIC columns. Columns with less than one monolayer follow an ANP mechanism. Reproduced with permission. ${ }^{31}$

This difference in retention mechanism is best understood by considering the impact on re-equilibration and salt concentrations required for increased retention. Since a diffuse water layer is a pre-requisite for HILIC, more column volumes are required to reequilibrate and generate this water layer to achieve reproducibility. In addition, salt concentrations must be increased in HILIC to affect increased retention, whereas the opposite trend is seen in ANP, less salt results in longer retention. Interestingly, ANP is capable of operating in either an RP like or ANP like mode depending on solvent concentrations. The dual retention mechanism possible in ANP is another differentiator between HILIC and ANP. ${ }^{32}$ Some challenges that both HILIC and ANP have had to overcome were the lack of commercially available column chemistries combined with the 
confusion regarding the retention mechanisms. Now that the distinction is clear, and as the column suite available for both of these techniques increases, the number of clinical applications for both ANP and HILIC is steadily growing. In subsequent sections, select applications of HILIC and ANP will be discussed as they relate to MMA method development.

\subsubsection{Ultra High-Pressure Liquid Chromatography Instrumentation}

The modern chromatographic approach is the UHPLC instrument, which is available from several manufacturers. The term ultra refers to the ability of these instruments to withstand back-pressures as high as 15,000 PSI. The capacity to operate at these pressures enables the use of small stationary phase particles, tightly packed with high MP flow rates. The benefit of small particles packed densely into columns is to increase surface area for chemical interaction, and a high flow rate enables a shorter analysis time without sacrificing efficiency. There are five typical components in a UHPLC instrument, including a sample manager, a column manager, a solvent manager, a detector, and a computer with software to operate the instrument.

The sample manager can be thought of as a refrigerated chamber that holds extracted samples in defined locations that can then be programmed into sample lists, which can be injected serially by an autosampler needle. The needle draws the sample into a sample loop and then toggles a valve to draw up the solvent behind the sample bolus and flush the sample out towards the column. The sample manager's primary purpose is to deliver the sample in a specific volume at the desired temperature. 
The column manager's role is to maintain the column at a fixed temperature, which will affect MP viscosity and other properties that can impact separation quality. The instrument's software will control the column manager. It is possible to hold several columns in modern instruments, which may be utilized in several different methods and can be toggled between using selections made in the sample list. The column manager's primary purpose is to hold the analytical columns that contain varied stationary phases in a constant temperature environment, signifying the physical location where separation occurs.

The solvent manager is the heart of the LC system, as it pumps the various solvents in precise quantities through the instrument. As mentioned previously, the solvent manager is used to provide a solvent to the sample loop to elute the sample bolus. In addition, the solvent manager delivers the precise percentages of MP A and B at the correct times as specified by the LC method, to achieve the desired gradient. The ability to fine-tune the percentage of weak or strong MP at any given point in the chromatographic run imparts a significant degree of chromatographic control. The solvent manager's primary purpose is to precisely mix and deliver a desired MP composition at the desired time to achieve reproducible chromatographic retention and separation of analytes on the column.

In theory, the detector is a somewhat interchangeable piece as many types have been used historically, including ultra-violet, evaporative light scattering and mass spectrometry, among others. However, for this project, MS detection is the only modality used, and the discussion will focus on this technology. MS is an incredibly 
complex and powerful technique that has evolved independently and in conjunction with GC and LC. MS will be discussed in the subsequent section in detail as an understanding of the scientific theory is critical in understanding the scope and application of the project. The detector's primary purpose is to detect, and when possible, quantitate the compounds eluting from the LC column. MS has the added advantage of providing a second dimension of data that can also help identify and add confidence to the identification, rather than relying on LC retention time alone.

The computer is a necessary component to perform data analysis. It often comes with proprietary software that can perform various functions from modulating instrument settings in a pre-programmed fashion to quantitation and data review. Before having a computer attached to the instrument with these capabilities, an operator would need to stand in front of the instrument and manipulate various dials at specific time points in the assay and then take paper printouts and analyze the data by some other method. As computing power and data analysis tools continue to advance, the control of the instrumentation and the ease of data manipulation continue to make the scientist's job easier. The primary function of the computer is to operate the instrument and assist in data analysis.

\subsubsection{Mass Spectrometry}

In the context of LC-MS/MS, the MS is simply referred to as the detector. However, this technique has a rich history and a host of applications that make it quite an exciting topic of discussion. The field of MS began at the turn of the $20^{\text {th }}$ century, around the same time that chromatography was discovered in the botany field. The first Nobel Prize 
for MS was awarded to J.J Thomson in 1906 in the field of physics. ${ }^{36-38}$ It several years for this technique that was popular in theoretical physics to migrate to the field of chemistry and find utility in research by quantifying components of a mixture. The transition into the clinical laboratory environment really began in the 1980s after the mandates Reagan rolled out as part of The Federal Drug Testing Program. ${ }^{22}$ The scope of mass spectrometry is vast, and applications so diverse it would be impractical to discuss the field's breadth. This section will discuss some basic definitions, instrument components, and the evolution of the MS in the clinical laboratory context. The progress from GC-MS to LC-MS/MS, and the various advances in instrumentation allowed this evolution to occur.

A mass spectrometer is an instrument that utilizes a gas beam of ions traveling in an electromagnetic field to measure the $\mathrm{m} / \mathrm{z}$ or mass to charge ratio of individual ionic species of a complex mixture. ${ }^{37}$ The basic information obtained from a mass spectrometer is a plot of $\mathrm{m} / \mathrm{z}$ ( $\mathrm{x}$-axis) versus abundance (y-axis), known as a mass spectrum. As all compounds have mass, measurement of anything is theoretically possible with mass spectrometry. The typical answers that mass spectrometry is used to find in analytical chemistry include what is in a sample and how much of each component. It is important to note that only charged compounds, ionic species, will be detected by a mass spectrometer. The driving force in the instrument is an electric or magnetic field, which does not affect neutrals.

The basic instrument schematic includes six components (Figure 9): sample inlet, ion source, mass analyzer, detector, vacuum, and computer. The individual components will 
be discussed to describe their purpose and basic operating principles. No attempt will be made to explain the breadth of possible variations that have been made to the instrument for varied applications, only select advancements as they pertain to the clinical laboratory and this research project.
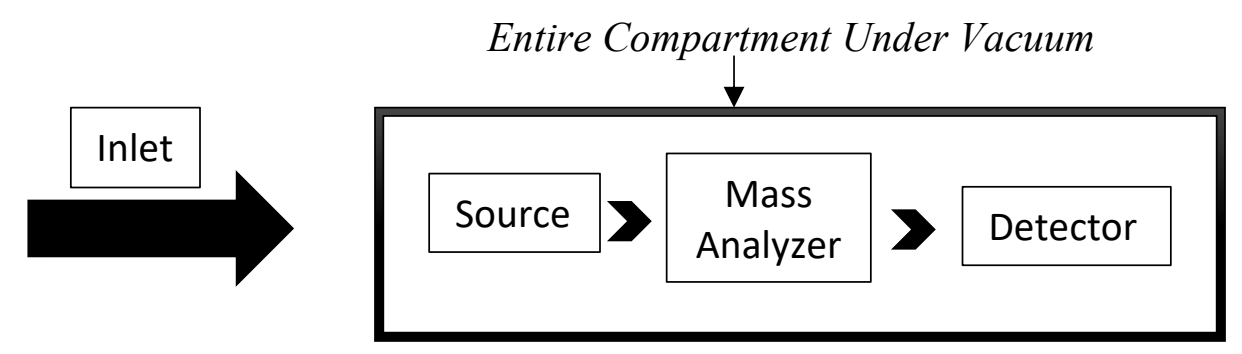

Figure 9. Schema of MS instrument. A simple diagram displaying parts of a mass spectrometer.

The inlet is the piece of the instrument where samples are introduced into the ion source. These samples need to be relatively pure, as a complex mixture can produce a very noisy and convoluted mass spectrum that will be difficult to interpret. There are numerous variations possible at the inlet; only three will be briefly touched upon herein. An infusion is a direct injection, typically using a small syringe, of a neat or pure solution into the mass spectrometer. Many instruments come with an infusion set-up on board. It is of great utility in early method development to tune the MS and observe the MS response to the compound without any other variables. Most clinical samples are not neat, but rather body fluids, which are complex mixtures and necessitate a different type of sample inlet. In the chromatography section, MS was described as the detector; however, in the MS section, chromatography and all the complexity in the science of separation that lies within is merely the inlet. 
Two standard sample inlets are GC and LC. GC was adopted earlier due to some apparent advantages, such as the compounds already being in the gas phase before entry into the ion source. Additionally, the instrumentation was widely available. However, GC-MS has its own set of limitations, and those will be discussed in subsequent sections. LC had been around for a long time before a few fundamental advances made it amenable for use as a sample inlet for LC-MS. The critical advances made to enable LCMS to become the success it is today, including advancements in ion source technology and the invention of the triple quadrupole $(\mathrm{QqQ})$. The primary goal of the sample inlet is to deliver a sufficient amount of sample into the MS for detection and quantitation of the ionic species therein.

The next three components discussed - the ion source, the mass analyzer, and the detector all exist under vacuum. The vacuum is critical because it removes room air and gases that could otherwise interact or collide with the ion beam and cause behavior that would not be expected based on the applied electric field. The stronger the vacuum, the internal space is less subject to any potential interfering molecules, resulting in a greater mean free path of travel for the incident ions. ${ }^{37}$ A typical LC-MS/MS instrument will have a rough pump that is further supplemented by a turbopump to achieve a strong vacuum, which lowers noise and increases resolving power. The primary purpose of the vacuum is to remove potential interactions inside the instrument so that only the mass analyzer can influence the trajectory of ions inside the ion beam.

The ion source has a role in producing charged species for introduction into the mass analyzer. There is considerable variation in ion sources. While most are inside the 
vacuum as previously described, some exist outside the vacuum as well but will not be discussed in this paper. To further understand the role of an ion source, a few chosen examples will be given, including hard and soft ionization techniques and familiar sources for GC-MS, LC-MS/MS, and TOF instruments. Hard ionization techniques are techniques where fragmentation is considerable, due to the high energy imparted on the molecule, such as in electron ionization (EI).

Soft ionization techniques involve minimal fragmentation. In some cases, no fragmentation occurs, but instead, an adduct is formed, which is the addition of weight through an ionic attraction of a charged species in the MP. An example of a soft ionization technique is electrospray ionization (ESI). The sample inlet provides a fine mist of droplets to the ion source, which is then gently evaporated and subsequently separated by a charge repulsion at the molecular level. In the case of hard or soft ionization, the standard result is an ionic species entering the mass analyzer. The preferred ion sources for GC-MS include EI and chemical ionization (CI). The advancements in the field that led to LC-MS success include significant advancements in ESI, which will be discussed in a subsequent section. The popular ion source for time of flight mass analyzers is the matrix-assisted laser desorption ionization (MALDI) source, which has performed very well for select microbiological and imaging applications..$^{22,36}$ The primary role of the ion source is to deliver ionic species into the mass analyzer in a charged state that is reproducible.

The type of mass analyzer used for this project is a QqQ detector, a scanning instrument. The principle of a scanning instrument, quadrupole, ion trap, or magnetic 
sector instrument, is that the electric or magnetic field must be continuously varied to obtain spectra. ${ }^{37}$ For comparison, a TOF instrument is an example of a non-scanning analyzer. Once an ionic species enters the mass analyzer of a scanning instrument, it is focused into an ion beam by an electromagnetic field that only allows ions that fit the selected $\mathrm{m} / \mathrm{z}$ ratio to reach the detector. The mass analyzer is responsible for the specificity possible in MS experiments as the detector is not able to distinguish what it is detecting. The mass analyzer's primary role is to vary the electromagnetic field in such a way that only the selected ion can achieve a stable trajectory and successfully reach the detector.

The detector and computer will simply be described as data collection and instrument control/data analysis, respectively. The detector types vary, but a popular type is electron capture detection, which is often coupled with electron multiplier technology, making the incident current recorded much greater. As previously stated, the detector does not record the identity of what is hitting it; instead, the mass analyzer directs only specific ions towards it, and the detector simply counts hits. The computer is used to operate the instrument and to analyze the data. One computer capable of controlling both systems, LC and MS, in one software platform is the current standard. The detector and computer's primary roles are to present the data in a meaningful way to the scientist and afford ease in operation through automation.

The potential of combining an instrument capable of robust and customizable separations, paired with a universal detector with high specificity, inspired the creation of a series of hyphenated devices. The most influential of the hyphenated instruments in the 
clinical laboratory include GC-MS for its early entry and broad applications with small molecules, followed by the diverse and influential LC-MS/MS. A brief review of the applications, utility, and limitations of these techniques will be discussed.

\subsubsection{GC-MS}

The use of GC-MS for the highly specific detection of small molecules in body fluids became mainstream in the late 1980s after a federal mandate. The increased utilization of GC-MS was a direct response to overcome some of the flaws inherent in immunoassay. ${ }^{39}$ The increased specificity and low limits of detection GC-MS could achieve quite remarkable for small volatile non-polar molecules that did not suffer from issues of thermal instability. ${ }^{22}$ The preparative chemistry required in extraction and sample preparation before the introduction onto the instrument is often cumbersome in GC-MS. Many biomolecules and drug metabolites are polar and require sample preparation steps such as derivatization to increase volatility or thermal stability for compatibility with GC-MS measurement techniques. The amount of time a scientist must spend preparing samples before injection can be a high cost to the laboratories operating budget while also requiring the use of harsh chemicals and large solvent volumes. Despite the time needed for adequate sample preparation, the requirement of volatility, and limitations regarding some molecules' thermal stability at high temperatures in helium gas, GC-MS is quite useful for some applications.

The GC-MS instrument is still a mainstay of the large modern clinical laboratory, with a strong history detecting small volatile non-polar analytes that have a high degree of thermal stability. Even with the 30 years of history that GC-MS has in the clinical 
laboratory, it remains a niche technique that many smaller labs do not have. The challenges that GC-MS poses which inhibit widespread application, include the complexity of the instrument, the sparsity of experienced personnel, the lack of standardized reagent kits and the specific requirements for the analyte, previously discussed. ${ }^{22}$ While the GC-MS instrument has shown substantial promise as a permanent resident of the clinical laboratory, it lacks the diversity of applications and reproducibility that LC-MS/MS has been able to achieve.

\subsubsection{LC-MS/MS}

The combination of LC and MS would take critical advancements in instrument technology, including the ESI source, the QqQ mass analyzer, and several decades to arrive as the premier instrument for separation and quantitation of a wide range of analytes. The significant advantage of LC-MS/MS techniques as compared to GC-MS is less restriction on target analytes, which results in less time in sample preparation for the scientist. The decrease in sample preparation time has positive impacts not only for budget but also for efficiency and turnaround time from order to result for the provider. The LC-MS/MS instrument does not utilize high temperatures to effect separation, as is the case for GC-MS, which allows LC-MS/MS to analyze thermally labile ionic species. Besides, LC-MS/MS instruments do not require volatility of the analyte, so large biomolecules can be assayed, higher than the 600 Dalton cutoff often cited in GC-MS literature. ${ }^{37}$ The time saved in sample preparation is a result of the LC-MS/MS lacking the two requirements as mentioned above of volatility and thermal stability, which often obviates the need for derivatization. These fundamental differences in analyte 
requirements between GC-MS and LC-MS/MS coupled with the vast diversity in column chemistries and MP combinations possible with LC-MS/MS have led to a boom in diverse applications using an LC-based platform.

The significant technological advancements that allowed LC-MS/MS to become a trusted application in clinical diagnostics were the ESI source and the QqQ detector. As discussed previously, the ESI source is a soft ionization technique that generates a fine mist of charged analytes inside droplets of the MP. As the droplets evaporate, their radii decrease, and the charged species contained within are forced closer together. At some critical point, the ionic species within are forced so closely together due to this gentle drying by sheath gas, that the electrostatic repulsion causes the droplet to break apart, known as a Coulombic explosion. ${ }^{37}$ The result of this explosion is that the ionic species is charged, in a gaseous state and free from the MP droplet. Before ESI technology, the volume of liquid leaving an LC column, could overwhelm the MS and prevent accurate measurement.

In addition, changes had to be made to typical LC MPs to be amenable to combination with MS. The high salt concentrations used in many LC-ultra-violet detection experiments needed to be adapted to work with MS. The salt would cause substantial dirtying of the ion-optics of the mass spectrometer. The standard MP additives that have been shown to work well in an LC-MS/MS experiment include low concentrations of ammonium acetate, ammonium formate, and formic acid. Ammonium acetate and ammonium formate are buffer salts that can stabilize the $\mathrm{pH}$ of MP that stay on the instrument for several days, and provide a charged adduct for use in an experiment 
with an ESI source. Recall that ESI is a soft ionization technique that typically does not fragment the molecular ion but can add an adduct mass to it. Formic acid is used to facilitate ionization and is typically added in both A and B MPs at concentrations between 0.1-1.0 percent. The advancements in ion source technology, column variety, and understanding of MPs that behave with MS were all significant steps. However, perhaps none of these compare to the creation of the QqQ (Figure 10).

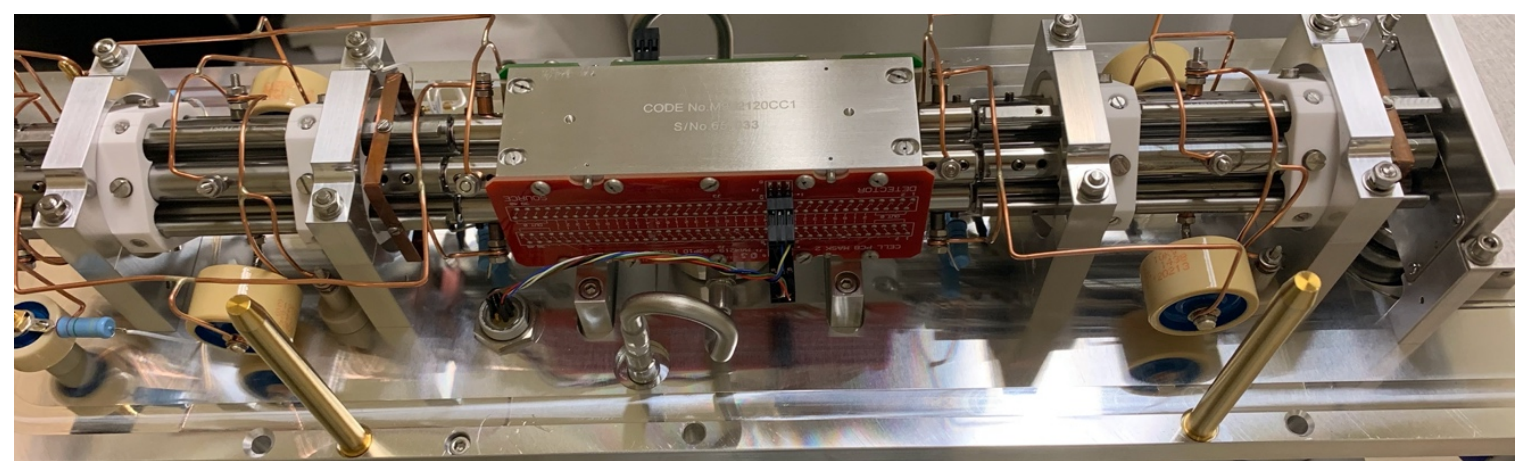

Figure 10. Triple quadrupole mass analyzer (QqQ). A photograph of a $\mathrm{QqQ}$ from a Waters Acquity TQD instrument.

The QqQ has made the LC-MS/MS assay a reference method for measuring many molecules due to its optimal specificity and quantitation accuracy. ${ }^{21,22}$ To be a reference method means that this assay has the profound distinction that all new tests by alternate methods be judged against this for accuracy. The QqQ was invented by Yost in the 1970s and has grown by leaps and bounds since then. ${ }^{40}$ The fundamental innovation in a QqQ compared to a single quadrupole instrument is that it is composed of two scanning mass analyzers in series, separated by a collision cell. The collision cell is filled with a noble gas, often argon, which does not interact chemically, yet provides an obstacle that results in collision-induced dissociation of ions as they travel through this cell at high speed. The resulting control of the mass spectrum measured is profound. 
The scientist can now select a specific $\mathrm{m} / \mathrm{z}$ in the first quadrupole, allow a fragmentation event to take place, and then select for a specific $\mathrm{m} / \mathrm{z}$ in the second quadrupole, which is known as measuring a transition. Another option is to select a specific $\mathrm{m} / \mathrm{z}$ in the first quadrupole, allow the fragmentation event to occur, then operate in scan mode and collect data on all fragments that pass through the second quadrupole. The result is a substantial increase in the specificity of the measurement, which translates to a more significant signal to noise ratio and ultimately a lower limit of detection for analytes in complex mixtures. ${ }^{21,22,37}$

The typical LC-MS/MS experiment involves multiple degrees of separation, extraction, then chromatography, then mass selection, followed by fragmentation and mass selection yet again. The use of hyphenated techniques has profound analytical power in that each step is a selective enrichment of the analyte in contrast to the background. The reality that each step in the process does not result in absolute analyte recovery but rather a selective increase in analyte over the matrix is essential to realize. This process results in an increase in the signal to noise ratio and imposes a challenge for quantitation relevant to the initial sample concentration. This challenge is met with the addition of internal standards for accurate quantitation. Internal standards will be discussed in a later section but are used to back-calculate the initial concentration from the measured concentration. The selectivity in each step of the separation is of utmost importance due to biological samples' inherent complexity and diversity. ${ }^{22}$ There is a limit to the amount of hyphenation that is useful, at some point, analyte recovery is so 
low that the signal to noise gains do not compensate for the absolute signal lost (Figure 11).

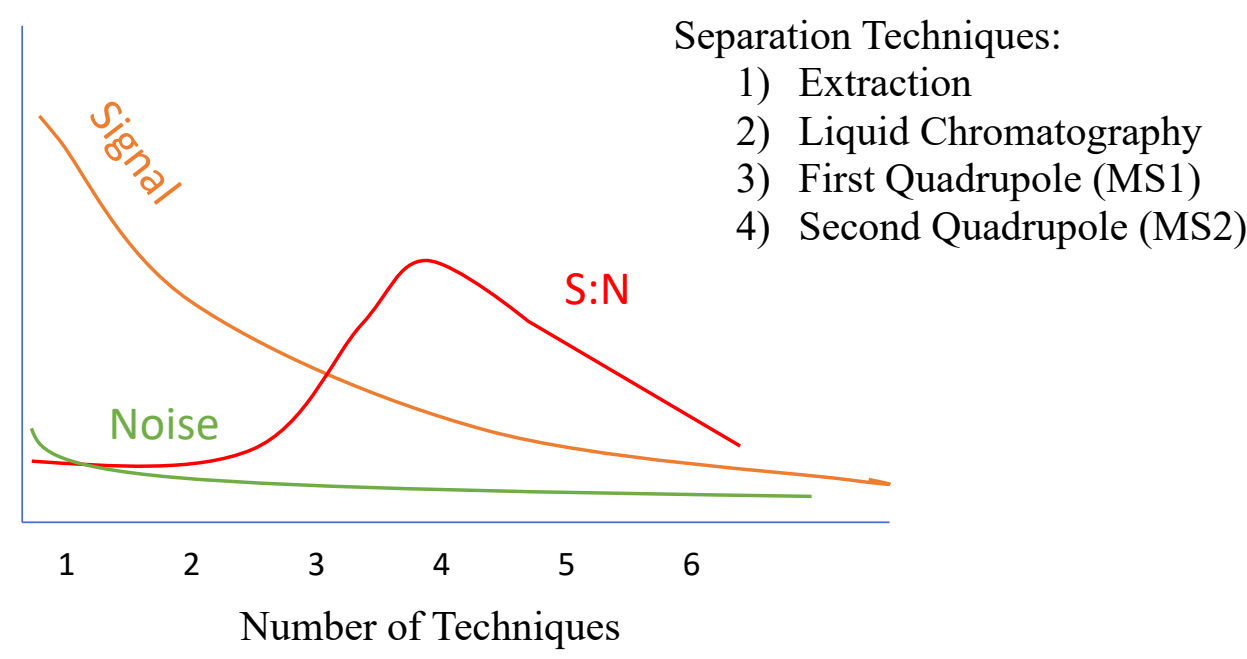

Figure 11. Selectivity gained by hyphenation. Hyphenation has a significant impact on the signal to noise ratio, up to a certain point. Adapted with permission from. ${ }^{41}$

The loss of analyte in each separation step precludes complete recovery but does allow for selective recovery. ${ }^{41}$ While the selectivity gains increase with each separation modality, there is an optimum number of separations. Excessive separations can inhibit the absolute recovery of the analyte, resulting in sensitivity issues for the detector. The ability to back-calculate the initial concentration relies on internal standards, critical to LC-MS/MS experiments. Internal standards are used not only to compensate for retention time drift as previously discussed but also to account for possible ion suppression and allow for accurate quantitation. ${ }^{41}$ An internal standard is typically an isotopically labeled analog to the analyte being measured, such that it behaves chemically similar but is distinguishable by the detector. ${ }^{37}$ Thinking back to the hyphenation example (Figure 11), an internal standard will theoretically be subject to the same percent 
recovery as the analyte and will function as a multiplier to adjust for weak recovery as the sample concentration is back-calculated.

Ion suppression is a concept that describes competition in the source for ionization energy, between the analyte and other unforeseen matrix components. The result of ion suppression is a relative decrease in energy imparted onto the analyte, which can be seen as a decrease in the signal measured at the detector. A common cause of ion suppression is phospholipids from plasma membranes eluting simultaneously in the chromatograph as the analyte of interest. Ion suppression can be checked in method development, with a specific infusion experiment, however since every patient sample has a unique quality to it, the internal standard provides a compensatory factor for these changes in ionization efficiency. $^{42}$

The advancements in LC-MS/MS instrumentation and experimental design make it the premier combined chromatographic-mass spectrometric technique in the clinical laboratory. The QqQ also has limitations, in that it only measures nominal mass and the breadth of chromatographic approaches that have been published are limited, despite theoretical potential. Despite the limitations, the QqQ is still the gold standard for quantitation of analytes in complex mixtures. Its utility will be highlighted in literature applications for the quantitation of MMA in subsequent sections.

\subsubsection{MMA in Literature}

The lack of an FDA approved commercial assay for MMA, does not mean that no research groups have successfully developed an assay for this compound. On the contrary, several groups have successfully measured MMA in biological samples by a 
variety of methods. MMA and its isomer SA structures pose particular challenges for techniques such as immunoassay, which have limitations for small molecules, to begin with, and would be plagued by cross-reactivity for this analyte pair. ${ }^{15,21}$ The polar nature of these molecules makes them less volatile, making sample preparation for analysis by GC-MS more cumbersome. However, the low molecular weight makes MMA a candidate for GC-MS or GC-FID measurement, as some groups have demonstrated. Measurement by LC-MS/MS removes the requirement for volatility, which, in theory, can reduce the sample preparation requirements before analysis. Within LC-MS/MS, different research groups have taken varied chromatographic approaches, including the standard RP-LC approach and a newer HILIC approach. The applications, challenges, and lessons learned from the work reported in the literature will be discussed.

\subsubsection{GC-MS for $M M A$}

In the last forty years, several groups have used gas chromatography for separation coupled with a flame ionization detector or mass-spectrometric detector to quantify MMA. MMA's challenge is the hydrophilicity and polarity of the molecule, often requiring extensive sample preparation and derivatization for amenability with GC separation. The variety of sample preparation techniques will not be covered exhaustively. However, several techniques will be discussed as a representative, including silyl, cyclohexanol, butanol, and chloroformate derivatives, to name a few. ${ }^{18}$

One of the earliest published methods for quantitation of MMA using GC was a capillary GC-MS method using $N$-methyl- $N(t$-butyldimethylsilyl)trifluoroacetamide to form $t$-butyldimethylsilyl derivatives of MMA. ${ }^{12}$ The Windelberg group published a 
derivatization scheme using dithioerythritol as a reductant, with ethanol to deproteinize and subsequent extraction with toluene and methylchlorofomate. ${ }^{43}$ The compounds measured by the GC-MS were the $\mathrm{N}(S)$-methoxycarbonyl ethyl ester derivatives of MMA, analyzed in selected ion monitoring mode (SIM). The requirements for derivatization were not unique to GC-MS detection; GC-FID analysis also required a derivatization scheme. Even though GC-MS adds specificity relative to GC-FID as the data has the second dimension of mass spectrum matching, other than merely retention time matching, a group has recently attempted to measure MMA with GC-FID. ${ }^{44}$

GC-MS is considered more sensitive and specific than GC-FID, however, when coupled with derivatization by trimethylsilyl trifluoroacetamide (TMS), quantitative analysis is still possible with FID detection. ${ }^{44}$ The method that achieved chromatographic separation for these TMS derivatives of MMA included a thirty-minute gradient and a final elution temperature of 290 degrees Celsius. While the lengthy gradient achieved reproducible results for the organic acid profile, the utility in a clinical setting is questionable as turn-around-time is a significant factor.

The GC-MS method for MMA that received the most use in a clinical setting was created by the Centers for Disease Control with a derivatization protocol involving cyclohexanol and subsequent formation of dicyclohexyl esters. ${ }^{45}$ However, the time for sample preparation and length of the chromatographic run prompted the CDC to migrate to an LC-MS/MS-based method to replace this aging assay. The work done by the CDC is a good case study for the use of GC-MS for MMA compared to LC-MS/MS's promise. Table 1 below shows some of the advantages of measurement on an LC-MS/MS 
platform, such as the time saved and related reduction in personnel costs. The typical requirement for MMA measurement by GC-MS is derivatization, which includes the use of harsh chemicals and increases sample preparation time. Many groups have been able to develop LC-MS/MS methods that have do not require derivatization. In addition to the reduced sample preparation times for LC-MS/MS, there is also a trend towards shorter chromatographic run times, which increases instrument throughput while decreasing turnaround time.

Table 1. LC-MS/MS vs. GC-MS for MMA (Adapted with permission. ${ }^{45}$ ) Sample preparation and analysis steps, comparing the CDC LC-MS/MS and GC/MS methods for MMA.

\begin{tabular}{|c|c|c|}
\hline Step & LC-MS/MS & GC/MS \\
\hline Sample volumettest, $\mu \mathrm{L}$ & 75 & 275 \\
\hline Calibration range, nmol/L & $25-2500$ & $0-20000$ \\
\hline Number of calibrators & 6 & 1 \\
\hline Specimen type & Serum & Plasma \\
\hline Derivative analyzed & Dibutyl ester & Dicyclohexyl ester \\
\hline Derivative used for analysis, (amount, $\mu \mathrm{L}$ ) & $3 \mathrm{M} \mathrm{HCl}$ in $\mathrm{BuOH}(125)$ & 1.5 M HCl in cyclohexanol (200) \\
\hline Derivatization time/temperature, $\min /{ }^{\circ} \mathrm{C}$ & 30160 & $15 / 115$ \\
\hline Sample extraction technique & Liquid-liquid extraction & Solid-phase extraction \\
\hline Sample preparation time, $\mathrm{h}$ & 4 & 12 \\
\hline Injection volume, $\mu \mathrm{L}$ & 3 & 1 \\
\hline Throughput (samples/run) & 160 & 36 \\
\hline Chromatographic run time, min & 6 & 15 \\
\hline
\end{tabular}




\subsubsection{LC-MS/MS for MMA}

The utility of LC-MS/MS compared to GC-MS, is obvious when it comes to a small polar molecule, such as MMA. The time saved in chromatography and sample preparation allows increased productivity from both the instruments and the scientists, making LC-MS/MS the obvious choice for measurement of this molecule and its isomer SA. However, the question of what LC separation mode to use is perhaps less obvious, and literature has no shortage of variations in this arena. In fact, there are more than fifteen analytical methods that boast different sample preparation and chromatographic approaches by LC-MS/MS. The mass transitions used to quantitate MMA in multiple reaction-monitoring mode are heavily dependent on derivatization reagents and are too cumbersome to describe exhaustively. The breadth of possibilities for transitions in multiple reaction monitoring (MRM) mode will only be displayed representatively in Table 2, as the main challenge with accurate quantitation of MMA is a chromatographic one. It should be noted in Table 2 that the choice of $\mathrm{m} / \mathrm{z}$ transitions is influenced by derivatization technique, ionization mode, and extraction technique. This table was utilized to confirm transitions discovered in method development experiments. 
Table 2. MMA MS/MS Values from Literature (Reproduced with permission. ${ }^{5}$ )

\begin{tabular}{|c|c|c|c|c|c|}
\hline Reference & Sample preparation & $\begin{array}{l}\text { LC run } \\
\text { time (min) }\end{array}$ & $\begin{array}{l}\text { Ions monitored } \\
\text { (1) MMA } \\
\text { (2) IS }\end{array}$ & $\begin{array}{l}\text { LLOQ } \\
(\mathrm{nM})\end{array}$ & $\begin{array}{l}\mathrm{HLOQ} \\
(\mathrm{nM})\end{array}$ \\
\hline Lakso et al. [8] & $\begin{array}{l}\text { Plasma }(0.2 \mathrm{~mL}) \\
\rightarrow \text { protein precipitation } \\
\text { (PPT) with acidified } \\
\text { acetonitrile }\end{array}$ & 10 & $\begin{array}{l}\text { Quantifier ions } \\
\text { (1) } 117.2 \mathrm{~m} / \mathrm{z} \\
\text { (2) } 120.2 \mathrm{~m} / \mathrm{z}\end{array}$ & $90^{\mathrm{a}}$ & 200,000 \\
\hline Blom et al. [11] & $\begin{array}{l}\text { Plasma }(0.1 \mathrm{~mL}) \\
\rightarrow \text { ultrafiltration } \\
\rightarrow \text { acidification }\end{array}$ & 5 & $\begin{array}{l}\text { Quantifier ions } \\
\text { (1) } 117 \rightarrow 73 \mathrm{~m} / \mathrm{z} \\
\text { (2) } 120 \rightarrow 76 \mathrm{~m} / \mathrm{z}\end{array}$ & No data & $2,000^{b}$ \\
\hline Kushnir et al. [12] & $\begin{array}{l}\text { Plasma, serum or [urine] } \\
\quad(1 \mathrm{~mL} \text { or [0.1 mL+0.9 mL water]) } \\
\rightarrow \text { liquid-liquid extraction (LLE) } \\
\text { with acidified methyl tert-butyl } \\
\text { ether (MTBE) } \rightarrow \text { derivatization } \\
\text { with acidified } n \text {-butanol }\end{array}$ & 1 & $\begin{array}{l}\text { Qualifier ions } \\
\text { (1) } 231 \rightarrow 119 \mathrm{~m} / \mathrm{z} \\
\text { (2) } 234 \rightarrow 122 \mathrm{~m} / \mathrm{z} \\
\text { Quantifier ions } \\
\text { (1) } 231 \rightarrow 175 \mathrm{~m} / \mathrm{z} \\
\text { (2) } 234 \rightarrow 178 \mathrm{~m} / \mathrm{z}\end{array}$ & 100 & 150,000 \\
\hline Schmedes and Brandslund [13] & $\begin{array}{l}\text { Plasma or serum }(0.75 \mathrm{~mL}) \\
\rightarrow \text { automated solid phase } \\
\text { extraction (SPE) } \\
\rightarrow \text { derivatization } \\
\text { with acidified } n \text {-butanol }\end{array}$ & 5.5 & $\begin{array}{l}\text { Quantifier ions } \\
\text { (1) } 231 \rightarrow 119 \mathrm{~m} / \mathrm{z} \\
\text { (2) } 234 \rightarrow 122 \mathrm{~m} / \mathrm{z}\end{array}$ & 48 & 200,000 \\
\hline Magera et al. [14] & $\begin{array}{l}\text { Plasma or [urine] } \\
(0.6 \mathrm{~mL} \text { or [0.1 mL]) } \\
\rightarrow \text { SPE } \rightarrow \text { derivatization } \\
\text { with acidified } n \text {-butanol }\end{array}$ & 3 & $\begin{array}{l}\text { Quantifier ions } \\
\text { (1) } 231 \rightarrow 119 \mathrm{~m} / \mathrm{z} \\
\text { (2) } 234 \rightarrow 122 \mathrm{~m} / \mathrm{z}\end{array}$ & $30^{c}$ & $16,700^{b}$ \\
\hline Fasching and Singh [15] & $\begin{array}{l}\text { Plasma }(0.2 \mathrm{~mL}) \\
\rightarrow \text { ultrafiltration } \\
\rightarrow \text { acidification }\end{array}$ & 3 & $\begin{array}{l}\text { Quantifier ions } \\
\text { (1) } 117 \rightarrow 73 \mathrm{~m} / \mathrm{z} \\
\text { (2) } 120 \rightarrow 76 \mathrm{~m} / \mathrm{z}\end{array}$ & 25 & 3,000 \\
\hline Pedersen et al. [16] & $\begin{array}{l}\text { Serum }(0.025 \mathrm{~mL}) \\
\rightarrow L L E \text { with acidified } \\
\text { MTBE } \rightarrow \text { derivatization } \\
\text { with acidified } n \text {-butanol }\end{array}$ & 4 & $\begin{array}{l}\text { Quantifier ions } \\
\text { (1) } 231 \rightarrow 119 \mathrm{~m} / \mathrm{z} \\
\text { (2) } 234 \rightarrow 122 \mathrm{~m} / \mathrm{z}\end{array}$ & $16^{\mathrm{c}}$ & $50,000^{b}$ \\
\hline
\end{tabular}

MMA's chromatographic challenge is due to its polar nature and an endogenous structural isomer that is highly abundant. A co-eluting isobar will prohibit accurate 
quantitation as the two mass spectrums will be indistinguishable, and the concentrations will be added together, making separation a requirement for useful measurement. The challenge of separating MMA and SA has been handled differently in various published analytical methods.

In theory, MMA's hydrophilicity makes it a poor candidate for RP-LC, yet many groups have tried this approach despite this fact. The reality that RP-LC is the dominant separation mode used in clinical laboratories and has the most significant market share of commercially available column chemistries often influences this technique's application, despite its shortcomings for polar molecules. ${ }^{18,26,46}$ The typical RP-LC approach uses a C18 column with long carbon chains that are chemically bonded to a silica particle and preferentially interact with non-polar molecules, causing retention. The typical gradient parameters include a water-based weak MP followed by a strong eluting MP with a higher percentage of strong organic solvents such as methanol or acetonitrile.

The purpose of RPLC can simply be described as the selective retention of non-polar compounds, which will elute in reverse order of polarity, with the most polar eluting first. The polarity of both MMA and SA means that they are both likely to elute early, causing issues with the background signal as most non-retained compounds elute early in a chromatographic run. Also, the structural similarity for two poorly retained compounds makes separating them by this technique difficult. For discussion, three distinct methods of dealing with this challenge by RP-LC will be compared, two of which involve derivatization and one of which does not. 
The most straightforward and most direct approach is to skip derivatization and analyze the MMA and SA directly, which allows for increased throughput relative to methods requiring derivatization. ${ }^{46}$ While this approach has a theoretical advantage in time saved, it is flawed in that it relies on a C18 column to efficiently separate two highly polar molecules, which are then measured in MRM mode using identical $\mathrm{m} / \mathrm{z}$ transitions. The obvious issue here is the high likelihood of co-elution, especially as the column ages and resolution decreases and the potential consequence of inaccurate quantitation. The theoretical time saved in sample preparation may well be consumed troubleshooting and repeating runs when this poor assay design does not function to its ideal capacity. The separation of MMA and SA without derivatization has been shown to achieve baseline resolution on an RP-LC column. However, it is likely to become more problematic as the column degrades with repeat injections. One group showed that every twenty injections, a significant column wash, with as many as one hundred column volumes of wash are needed to prevent deterioration in peak shape on a C18 column. ${ }^{5}$

The remainder of the approaches for RP-LC method development for MMA all involve derivatization. However, the combinations are extensive and will only be briefly described herein. The approaches for RP-LC with derivatization typically fall into two categories, derivatization to improve separation by RP-LC or derivatization that creates a unique mass spectrum between the two isomers, SA and MMA., 2,7,16,18,47 While most approaches use chromatography to separate these two isobars, one group developed an interesting approach in which derivatization precluded the need for separation. ${ }^{2}$ The mass spectra of MMA and SA without derivatization are identical, but when butylated the 
mass spectra are distinct. ${ }^{2}$ The two compounds are distinguished by a branching ratio used to calculate the amount of each isomer. This approach is an interesting one in that it does not rely on the RPLC to separate these two compounds but instead uses the column as a simple sample inlet and lets the MS/MS distinguish the two by mass spectrum.

The vast majority of derivatization schemes used in literature for MMA detection by RP-LC are performed to improve the separation. The creative ability to use derivatization to circumvent the need for separation by LC does not reduce the sample preparatory requirements or use LC effectively. Nevertheless, Kushnir's approach is unique compared to the standard attempts to derivatize to effect a greater separation by LC. As an example, one group reported a chromatographic resolution of greater than ten or 0.81 minutes of separation between the isomers MMA and SA in an RP-LC run with derivatization. ${ }^{47}$ This is a promising result, but the length of time required for this analysis is eight minutes, and the cost per test is considerably higher with derivatizing reagents involved. ${ }^{48}$

The separation of SA and MMA by RP-LC can be aided by derivatization, including esterification of the carboxylic acid moieties. Many techniques exploit the pKa difference between SA and MMA with a MP pH around 4-4.5, showing the most efficient separation. ${ }^{47}$ Other groups have tried solid-phase extraction with positive pressure devices, which are cumbersome and require additional equipment. ${ }^{18,47}$ Although there have been many groups to publish methods using RP-LC-MS/MS, the requirement for derivatization to achieve an accurate and robust separation make this a less than ideal solution to the chromatographic challenge posed by these isobars. RP-LC, although 
coupled with costly derivatization and laborious sample preparation, it is also trusted and widely used due to inertia in the field. ${ }^{48}$

A few enterprising groups are starting to look into orthogonal separation techniques, which allow for robust separation without the drawbacks previously mentioned using RPLC for polar molecules. The most widely published of these orthogonal separation modes is HILIC, a chromatography form that retains polar compounds by a partition mechanism. The mode of retention in HILIC is particularly well suited for retaining the polar MMA and SA, which would elute later in the gradient, with non-polar molecules eluting earliest. ${ }^{26,48,49}$ The increased retention time, results in a more reliable separation without the need for derivatization to enhance the interaction with the stationary phase. While it may seem common knowledge that utilizing a stationary phase that retains polar molecules preferentially will enhance the efficacy of a separation, there were many myths in the field regarding the incompatibility of these LC modes with detection by MS. In other words, RP-LC was considered favorable because it is thought to be the most compatible with MS detection. The somewhat misguided concerns with HILIC that reinforced this reliance on RP-LC included the excessive column volumes required to reequilibrate a HILIC column, the high aqueous content of the MP's incompatibility with an ESI source and the lack of commercially available stationary phases.

In some pioneering work on MMA done in 2011, separation of MMA using HILIC-MS/MS was shown to be a robust technique with excellent chromatographic resolution for MMA, SA and phospholipids. ${ }^{26}$ This work was a significant step away from RP-LC as the only option in the theoretical separation space available to clinical 
laboratorians. The publication of this HILIC-MS/MS method for the quantitation of MMA successfully debunked several myths. The work demonstrated a high throughput method that did not require excessive re-equilibration time for reproducible chromatograph. It demonstrated the divert valve's utility for preventing the high aqueous content portion of the run from flooding the source. The final method achieved in this pioneering work by Rappold and Grant had a total run time under three minutes and reproducible chromatography at ten thousand injections. ${ }^{26}$ Overall, the work highlighted the potential for utilizing non-traditional LC-MS/MS MP to achieve excellent chromatographic control for polar molecules.

HILIC is not the only orthogonal separation mode available, but it is the most widely used based on current literature for MMA. There was some lack of clarity in the field over the distinction between HILIC and ANP. Some considered the former a subdivision the latter, others describing their difference based on retention mechanism and another group showing the heterogeneity within this stationary phase class when it comes to adsorbed water layers. ${ }^{26,31,35,50}$ The accumulation of these examples over the last decade or so paint a clearer picture; HILIC columns operate on partition mechanism, with the thickness of the water layer varying incredibly from column to column. ${ }^{31,35}$ HILIC columns are made with polar functional groups bonded to Type-B silica beads, which have silanols (Si-OH) on the surface and encourage the formation of that water layer (Figure 7). Whereas ANP columns are made with Type-C silica beads, which are covered in silica hydride moieties preventing the formation of this water layer. ${ }^{50}$ 
ANP columns have less than a monolayer of adsorbed water on their surface, which supports the adsorption mechanism proposed by the Pesek group. ${ }^{31,35}$ The work by Soukup also demonstrated vast heterogeneity in the thickness of the adsorbed water layer, which explains the dramatically different ideal conditions for each column, as found by Rappold and Grant in their work with HILIC method development for MMA. ${ }^{26,35}$ The preliminary work has been done to prove orthogonal separation modes to RP-LC have a place in the clinical laboratory, but what has yet to be done is a side by side comparison of a HILIC and ANP retention mechanism for the quantitation of MMA.

\subsubsection{Research Goals}

This study aims to perform preliminary experiments that will later guide the method development process for a quantitative LC-MS/MS assay for MMA in biological samples. The approach can be broken down into three fundamental sections: (1) to develop an MS/MS method for MMA, MMA-d3 and SA*; (2) to develop and optimize an LC method; and (3) to compare the separation modes ANP and HILIC for MMA.

In this study, MMA, MMA-d3, and SA* were infused directly into the MS/MS, and a variety of instrument parameters were adjusted to identify target ions representative of each compound. Once the target ions were found, optimizing tune settings was performed to set appropriate temperatures and voltages for maximum response. In addition to identifying stable representative target ions, the aim of the MS method development portion of the study included identifying daughter ions and finding the ideal ionization mode. Another aim of the study was to choose optimal resolution settings and verify the detector's mass accuracy. 
The study's second aim was to develop and optimize an LC separation method across three stationary phases to determine the ideal column chemistry and separation mode for MMA, MMA-d3, and SA*. Taking the MS/MS transitions from the first part of the study, a preliminary method was developed to experiment with various LC gradients with subsequent detection by MS/MS. The three stationary phase classifications tested included RP, ANP, and HILIC. For each classification, one representative column was tested, Phenomenex Kinetex XB-C18, Cogent DH, and Waters Acquity UPLC® BEH Amide, respectively. Optimization of parameters such as flow rate, MP composition, concentrations of various mobile phase additives, and the gradient slope was performed to improve peak shape, retention time and examine effects to selectivity. In addition to measuring the influence on chromatographic parameters, the various separation modes and stationary phases were compared with elution time, mobile phase composition at that elution time and effect on the signal generated by the MS/MS.

The study's third aim was to examine if the retention of MMA, MMA-d3, and SA* on the Cogent DH column versus the Waters BEH Amide column exhibited an obvious ANP versus HILIC retention mechanism. In order to test this theory, an experiment involving salt concentration was performed. The salt concentration study was performed by varying the concentration of buffer salt ammonium acetate from $10 \mathrm{mM}$ to $100 \mathrm{mM}$ and observing effects on retention time across the two previous columns. 


\section{MATERIALS AND METHODS}

\subsection{Materials}

\subsubsection{Analytes}

The analytes were chosen as follows: MMA is the target analyte that predicts the pathophysiology, MMA-d3 is an internal standard for aid in quantitation, and to account for possible ion suppression, and SA is an endogenous interferant. However, as mentioned previously, 2,2-dimethylsuccinic acid (SA*) was used in place of SA in this study due to limitations in availability. The analytes of interest were obtained from Cerilliant, with MMA and MMA-d3 having initial concentrations of $1 \mathrm{mg} / \mathrm{mL}$ in acetonitrile, whereas $\mathrm{SA}^{*}$ was in pure powdered form. The formula weight for MMA is given as $118 \mathrm{~g} / \mathrm{mol}$. The formula weight for MMA-d3 is given as $121 \mathrm{~g} / \mathrm{mol}^{\text {. SA*'s }}$ formula weight is 146 , which is different from the actual analyte of interest, which is SA with a mol weight of $118 \mathrm{~g} / \mathrm{mol}$. SA* is used as a surrogate for SA but still has utility in measuring chromatographic separation effects. The SA* was prepared by measurement on a Denver balance and then dissolved into $\mathrm{MeOH}$ to achieve a final concentration of 1 $\mu \mathrm{g} / \mathrm{mL}$. For most experiments described below, a concentration of $1 \mu \mathrm{g} / \mathrm{mL}$ in $\mathrm{MeOH}$ was used for all three analytes of interest. However, some infusion experiments were attempted at concentrations of $500 \mathrm{ng} / \mathrm{mL}$. The desired concentration was achieved by

diluting this $1 \mu \mathrm{g} / \mathrm{mL}$ solution in $\mathrm{MeOH}$ with an equal part $\mathrm{MeOH}$ with $0.2 \%$ formic acid to achieve a final concentration of $0.1 \%$ formic acid. The vendor information can be found in Table 3. 
Table 3. Analyte Vendor Information

\begin{tabular}{|c|c|c|c|}
\hline Name & Vendor & Catalog Number & Lot Number(s) \\
\hline Methylmalonic Acid & Cerilliant & M-080-1ML & $\begin{array}{c}\text { FN05101902 } \\
\text { FN048181405 }\end{array}$ \\
\hline Methylmalonic Acid -d3 & Cerilliant & M-105 & FN09171501 \\
\hline 2,2 dimethylsuccinic acid & Sigma Chemical & D-3394 & 54H3673 \\
\hline
\end{tabular}

The dicarboxylic acid structure of the analytes makes them polar molecules with two pKa's. The two pKa's of MMA are at pHs of 3.07 and 5.76, while the pKa's of SA are at pHs of 4.21 and 5.64, both at 25 degrees C. ${ }^{20}$ While no information is available regarding the pKa's of MMA-d3 they can be assumed to be quite similar to MMA, as the only difference is the replacement of three deuterium ions for three hydrogens. The slight difference in pKa's is a characteristic that can be exploited in separation if carried out at a carefully chosen $\mathrm{pH}$.

The molecular formula of the analytes can be seen in Table 4.

Table 4. Analyte Molecular Formulas

\begin{tabular}{|c|c|}
\hline Name & Molecular Formula \\
\hline Methylmalonic Acid & $\mathrm{C}_{4} \mathrm{H}_{6} \mathrm{O}_{4}$ \\
\hline Methylmalonic Acid $-\mathrm{d} 3$ & $\mathrm{C}_{4} \mathrm{H}_{3} \mathrm{O}_{4} \mathrm{D}_{3}$ \\
\hline Succinic Acid & $\mathrm{C}_{4} \mathrm{H}_{6} \mathrm{O}_{4}$ \\
\hline 2,2 - dimethylsuccinic Acid & $\mathrm{C}_{6} \mathrm{H}_{10} \mathrm{O}_{4}$ \\
\hline
\end{tabular}

As mentioned previously, the ideal analyte to test chromatographic separation for the two isomers would have been SA rather than dimethylsuccinic acid (SA*). However, the 2,2-dimethylsuccinic acid was procured before the experiment's initiation and as merely an interferant in the assay, not a true analyte of interest; it was decided that the procurement of SA was an unnecessary cost. While there are obvious disadvantages 
making assumptions about SA's behavior based on 2,2-dimethylsuccinic acid, it provides some information regarding chromatographic control of a succinic acid derivative.

\subsubsection{Mobile Phase Constituents}

This study's MP constituents include both aqueous and organic solvents, buffer salts, and formic acid. The MPs were prepared by weighing the buffer salts on the A-250 analytical balance. Subsequently, those salts were dissolved in the desired proportions of aqueous and organic solvents to achieve the concentration required in that portion of the experiment. All MPs were made with dedicated LC-MS/MS glassware that was cleaned with a protocol to prevent detergent contamination or other potential interferants. The $\mathrm{pH}$ of select MPs was checked using a Corning $320 \mathrm{pH}$ meter. All MPs prepared were sonicated with a loose cap for ten minutes in a Branson Ultrasonic Cleaner sonicator to ensure that they were degassed before loading onto the LC-MS/MS. All reagents were HPLC grade. The water used in the study was treated by a Millipore Milli-Q water system, which produces $>10 \mathrm{mOhm}$ water, which was subsequently polished by a Barnstead filtration device to reach a final resistivity of $18.2 \mathrm{mOhm}$. The list of MP constituents used in this study can be found in Table 5 .

Table 5. Mobile Phase Constituents

\begin{tabular}{|l|c|c|}
\hline \multicolumn{1}{|c|}{ Name } & Vendor & Lot Number \\
\hline 18.2 mOhm water & Barnstead EasyPure RF & - \\
\hline Acetonitrile & J. T. Baker & 0000242659 \\
\hline Methanol & Honeywell & DW224-US \\
\hline Ethyl Acetate & J. T. Baker & 0000240898 \\
\hline Ammonium Acetate & Sigma-Aldrich & BCBT2624/BCCB9847 \\
\hline Ammonium Formate & Sigma-Aldrich & BCCC3106 \\
\hline Formic Acid & Fisher Chemical & 195095 \\
\hline
\end{tabular}




\subsubsection{Columns}

An analytical column is composed of small silica beads packed into stainless-steel housing, with frits on either end to contain column's beads. The silica beads often have various functional groups bound to their surface to affect the desired selectivity for specific classes of analytes. The three columns used for this experiment are each representative of a unique chromatographic separation mode. The C18 column was chosen to represent a stationary phase that is amenable to RP separation. The Cogent DH column was chosen to represent a stationary phase that is amenable to ANP separation. The Amide BEH column was chosen to represent a stationary phase that is amenable to HILIC separation. The characteristic functional group present on each of these columns can be seen in Figure 12 below.
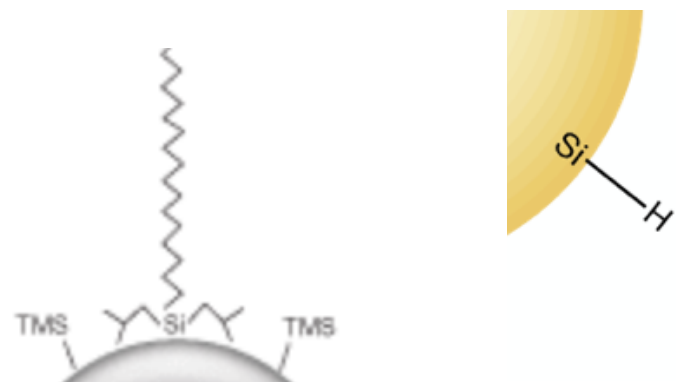

Figure 12. Column chemistries for $\mathrm{C} 18$ vs. DH vs. Waters Amide BEH. Representative imaged of the surface chemistries for the particles within each column used in the experiment. Phenomenex C18 can be seen on the left, Cogent DH in the center, and Waters BEH-Amide column on the right.

The clinical environment trend is to use smaller internal diameter columns with tiny particles, which together decrease solvent usage and maximize surface area for potential 
contact of the analyte with the functionalized stationary phase. There is a tradeoff between chromatographic resolution and chromatographic run length, where column length, flow rate, and back pressure are all factors. Generally speaking, a shorter column will have lower back pressure and higher throughput than a longer column at the same flow rate, but chromatographic resolution may be negatively impacted. Suppose the instrument can operate with high back pressure. In that case, as is the case for a UHPLC instrument, a potential solution is to increase the MP flow rate to achieve suitable chromatographic resolution within the desired run time. The column dimensions used in this experiment can be seen in Table 6. It should be noted that the $\mathrm{C} 18$ and DH columns are the same lengths, but the BEH-Amide column is twice the length. A longer column can offer more theoretical plates, which can translate to better resolution but also longer run times or higher back pressures can present challenges as will be discussed later.

Table 6. Column Dimensions

\begin{tabular}{|l|l|l|l|l|}
\hline Column & Manufacturer & Dimensions & Particle Size & Pore Size \\
\hline Kinetex XB-C18 & Phenomenex & $50 \times 2.1 \mathrm{~mm}$ & 2.6 micron & $100 \AA$ \\
\hline Diamond Hydride & Cogent & $50 \times 2.1 \mathrm{~mm}$ & 4 micron & $100 \AA$ \\
\hline BEH-Amide & Waters & $100 \times 2.1 \mathrm{~mm}$ & 1.7 micron & $130 \AA$ \\
\hline
\end{tabular}

\subsubsection{Miscellaneous Materials}

In addition to the analytical instrument discussed in the next section, several ancillary pieces of laboratory equipment were used in various reagent preparation phases. The equipment list will be exhaustively prepared in Table 7, and a short description of the practical usage will follow. 
Table 7. Additional Lab Equipment

\begin{tabular}{|c|c|c|}
\hline Equipment & Vendor & Function \\
\hline Analytical Balance & $\begin{array}{c}\text { Denver Instrument Co. } \\
\text { (A-250) }\end{array}$ & Weigh MP additives and SA powder \\
\hline $\begin{array}{c}\text { Lab Grade Water } \\
\text { Filter }\end{array}$ & Millipore & $\begin{array}{c}\text { Generate }>10 \text { mOhm water for } \\
\text { further filtering }\end{array}$ \\
\hline $\begin{array}{c}\text { LC-MS Grade Water } \\
\text { Filter }\end{array}$ & Barnstead & $\begin{array}{c}\text { Generate }>18 \text { mOhm water for MP } \\
\text { preparation }\end{array}$ \\
\hline $\begin{array}{c}\text { Sonicator } \\
\text { Laboratory } \\
\text { Glassware }\end{array}$ & Branson Ultrasonic \\
Cleaner & Degassing of MP's \\
\hline pH meter & Schott & $\begin{array}{c}\text { To measure and hold MP and stock } \\
\text { standard solutions }\end{array}$ \\
\hline Pipette & Corning 320 & To pH MP \\
\hline
\end{tabular}

The various equipment listed in the table above was used to prepare standards and MP as briefly described below. The analytical balance was used to weigh the 2,2 dimethyl succinic acid and the buffer salts for the various MPs. Many of the MPs required water, and the process for the preparation of LC-MS grade water involved two filtration units, as described in section 2.1.2. Working standards were made by spiking stock standards into an appropriate volumetric flask filled halfway with methanol using a hand pipettor of appropriate volume and then filling with methanol up to the line. The $\mathrm{pH}$ meter was used to $\mathrm{pH}$ the MP used for infusion experiments to predict MMA's ionization state.

\subsection{Instrumentation}

The LC-MS/MS instrument used in this study was a Waters-Acquity TQD. The instrument is equipped with a sample manager, a binary solvent manager, a column oven, a computer, an ESI source, and a tandem MS (Figure 13). 


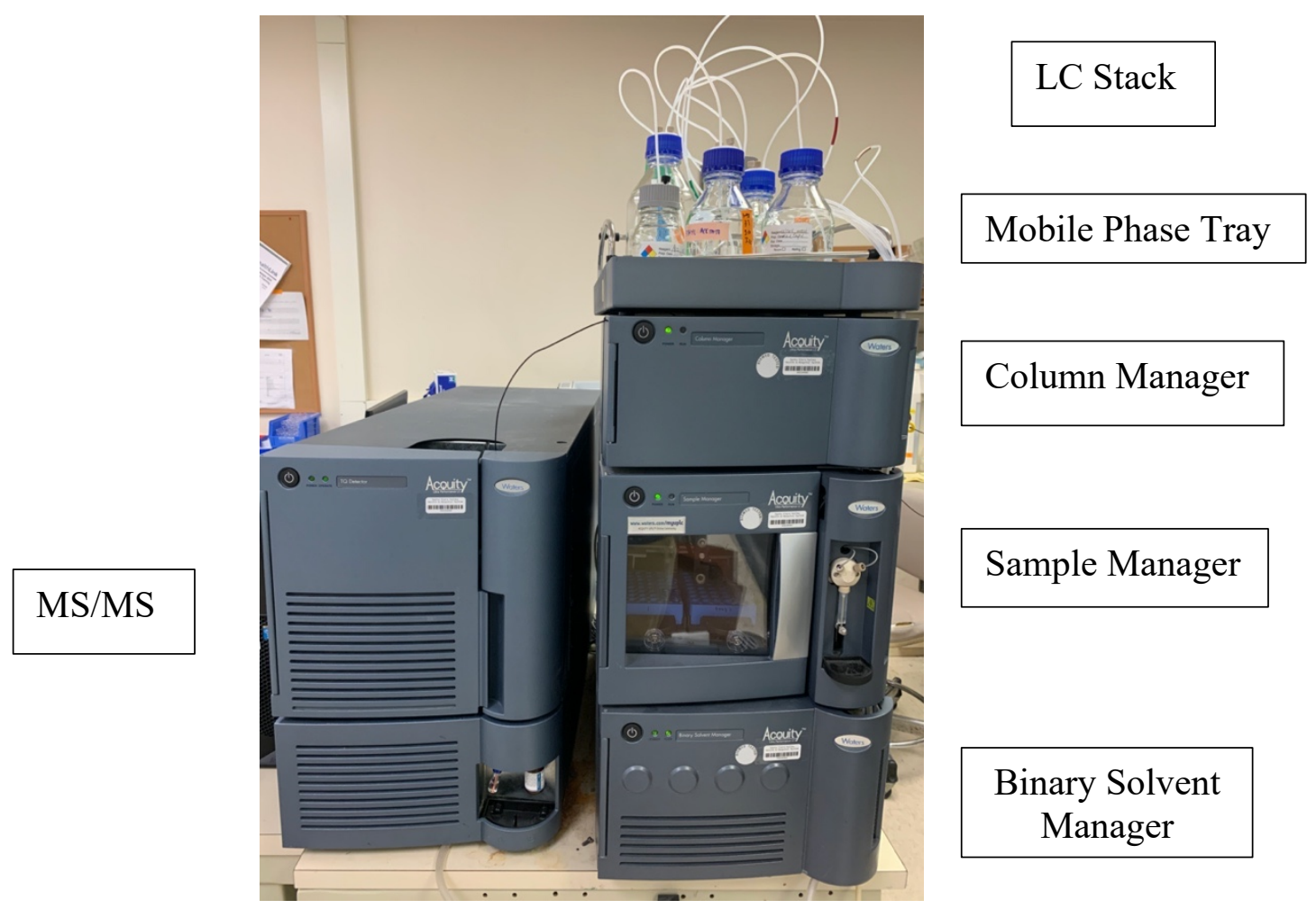

Figure 13. Waters Acquity TQD used in this experiment. Photo of the instrument all experiments were performed on - various modules labeled for clarity.

The sample manager is in a temperature-controlled environment with an autosampler needle capable of serial injections by programming a sample list on the interfaced computer. The autosampler needle draws a volume specified by the method into a sample loop attached to a divert valve. The divert valve then actuates to accept fluid from the binary solvent manager, which flushes the sample bolus into PEEK tubing and moves towards the analytical column housed inside the column oven.

The binary solvent manager has two pumps for each MP (A or B, respectively), which drive the high precision fluidics system on board and prevent any turbulence or interruptions to flow otherwise possible if the pump was inconsistent. The binary solvent manager delivers an exact MP composition as specified by the operator in the method. 
The two A and B side MP pumps to mix the MPs and deliver a constant flow rate to the analytical column. The binary solvent manager also has an online degasser, removing small air bubbles to prevent air from getting trapped inside the column.

The analytical columns are housed within a temperature-controlled column oven, which holds a consistent temperature throughout the analytical run. This instrument can hold four analytical columns, and the operator can easily toggle through the desired column for each application as specified in the method. The sample bolus is separated and concentrated into various peaks based on the analytical column's selectivity in use and eluted into a short length of PEEK tubing traveling towards the MS.

The PEEK tubing coming from the column oven then feeds into another divert valve, which can toggle towards waste or the MS depending on the operator's specifications in method development. Another potential path to the MS is through an infusion syringe that skips the LC and directly feeds into the source for tuning and method development experiments. The path into the MS includes traveling through an ESI probe, which is equipped with a capillary needle that is charged and produces a fine spray of mist with sample and solvent present. The sample now in a mist in the gas phase is dried with a gentle stream of nitrogen gas supplied as a sheath gas. The sheath gas helps desolvate the analyte while preventing the deposition of particulates on the skimmer cone. The charged analytes then enter into the MS by passing through a charged capillary cone and entering what is often referred to as $\mathrm{Q}_{0}$, or the beginning of the ion optics.

Inside the MS/MS, the ions are first focused into a beam by applying an electromagnetic field. The two quadrupoles can be operated in several ways, but for the 
method developed for this study is an MRM method. In an MRM method, the first quadrupole selects for an ion of interest, fragments it in the collision cell by a collision-induced dissociation with argon gas. The fragments can then be selectively allowed to pass to the detector by applying another electromagnetic field, which allows for an unparalleled level of specificity. The detector measures the current created by ions impacting its surface, and this signal is converted and displayed as a quantitative measurement in the MassLynx (v4.1) software package.

The operator can perform various functions inside the MassLynx software, including but not limited to peak review, signal to noise quantitation, calibration, and quantitation of analytes. MassLynx was used to develop the various versions of methods that were used, and sample lists were created to serially inject through a range of conditions, with subsequent data review being performed in MassLynx as well.

\subsection{Methods}

\subsubsection{Development of an $M S / M S$ method for $M M A, M M A-d 3$, and $S A *$}

The identification of target ions, fragment ions, and the appropriate MS acquisition parameters was obtained by a series of experiments involving the direct infusion of pure standards into the MS. The infusion was performed in two ways: a direct infusion and a combined infusion, which involved the standard being infused blending with the LC eluent in the ESI source. The flow rate for the infusion syringe delivering the compounds, MMA, MMA-d3, and SA*, was 10-20 $\mu \mathrm{L} / \mathrm{min}$, regardless of combined or direct infusion. The MP used for combined infusion was composed of 85 percent acetonitrile and 15 percent water, with $100 \mathrm{mM}$ ammonium acetate and one percent 
formic acid at a flow rate of $800 \mu \mathrm{L} / \mathrm{min}$. In addition to observation for expected target ions related to the analyte, based on the compound's formula weight and the ionization mode used, the span was increased to observe potential contaminants and see if there were any unexpected in source fragmentation taking place. Adjusting the span essentially alters the amount of the mass spectrum visible to the operator allowing one to zoom in or observe a more significant portion of the $\mathrm{m} / \mathrm{z}$ range.

A typical infusion set-up can be seen in Figure 14.

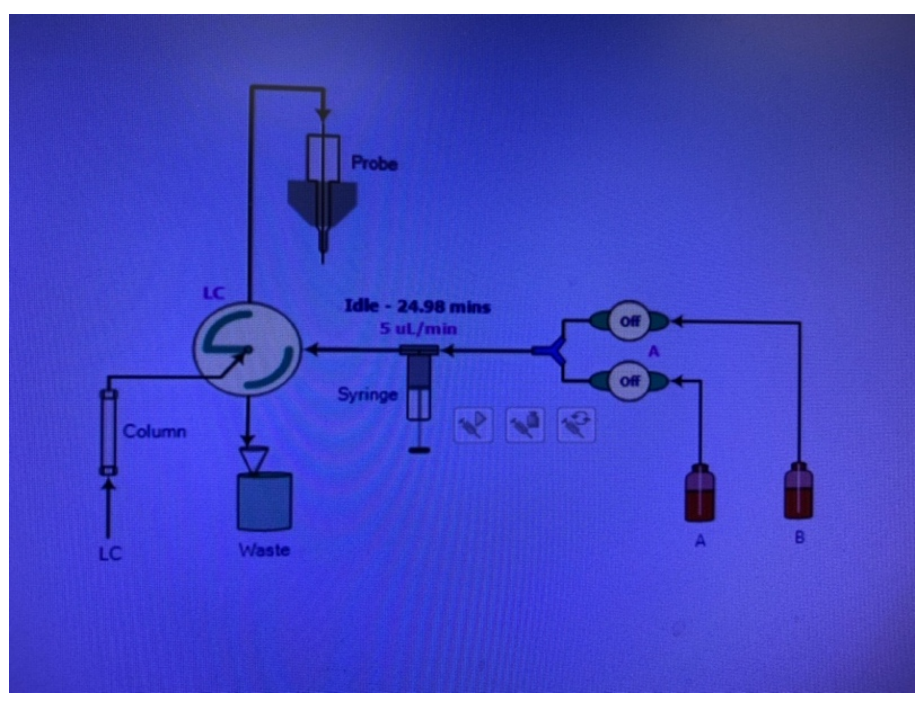

Figure 14. Infusion set-up. An image from Waters MassLynx software showing a live feed of the infusion set-up.

\subsubsection{Identification of Precursor Ions}

Two approaches were taken while infusing the compounds, to identify precursor ions for the target analytes. Waters MassLynx software comes equipped with a program called Intellistart, an automated optimization program that varies the MS acquisition parameters if a specific precursor ion is specified. In theory, Intellistart should return fragment ions and all relevant tune parameters that are needed to optimize signal 
response. The utility of this program will be discussed further in the Results section, however in short, it was not entirely useful as the results obtained varied considerably each time it was performed, and it often returned precursor and fragment ions that did not make sense.

The method that did work to identify precursor ions was a direct infusion combined with manual tuning. The initial concentration of analyte infused was $500 \mathrm{ng} / \mathrm{mL}$ in methanol. However, due to the presence of some contamination in the sample reservoirs feeding into the ESI source, the background was substantial at these concentrations. The concentration of analyte that resulted in a successful infusion experiment was $1 \mu \mathrm{g} / \mathrm{mL}$ in methanol. The basic procedure involved modifying various MS tune parameters while constantly infusing the analyte of interest and observing the effect on the response, with the maximization of the response being the goal. The optimized parameters included capillary voltage, cone voltage, collision energy, MS1, and 2 resolution and ionization mode.

The process to identify precursor ions included turning the collision gas off, and the collision energy was set to zero. The capillary voltage was then incrementally increased from zero up towards the maximum, and the maxima in response for the analyte signal was determined at a specific capillary voltage. The capillary voltage was then held constant at the voltage resulting in the signal maxima, and the cone voltage was optimized in the same fashion. These optimization experiments were carried out in both ionization modes, ESI positive and ESI negative modes. The two ionization modes were compared for optimal signal height for the analyte and compared the background 
spectrum's noise. The ionization efficiency was superior in ESI negative mode, and the background mass spectra had significantly fewer interferants, perhaps due to a contamination issue, as will be discussed in the Results section. The choice to use unit resolution, versus low resolution, versus quantitative resolution was made empirically based on the maximum signal obtained in any of the three settings. The implications of this will be discussed in the Results section.

\subsubsection{Identification of Fragment Ions}

Once the correct precursor ions were detected, and their signal strength optimized, the iterative process was repeated for the fragment ions. As may be expected, the fragments produced and those fragments' intensity will be highly dependent on the tune parameters, namely collision energy and cone voltage. The process for manually tuning for fragments is analogous to precursors' process, except that collision gas is turned on. The MassLynx software allows the separate quadrupoles MS1 and MS2 to be operated in either a scanning or selective mode. In order to scan for fragment ions, the MS1 was set to allow only the precursor ion of interest to pass through, and the MS2 was left in a scanning mode where it would record the $\mathrm{m} / \mathrm{z}$ of all fragments generated by this precursor ion.

Once a stable fragment was observed, the span was then reduced to focus on the specific fragment ion. Optimization of the MS acquisition parameters were performed to optimize the signal for this transition. A transition is defined as a precursor ion paired with a fragment ion in an MS/MS experiment. The first quadrupole selects for the precursor only, and the second quadrupole selects for only the fragment ion of interest. 
Generating one signal from the transit of this ion from MS1 through the collision-induced dissociation in the collision cell and selecting only one of the fragment ions in MS2. The general scheme for the measurement of an MRM transition can be seen in Figure 15.
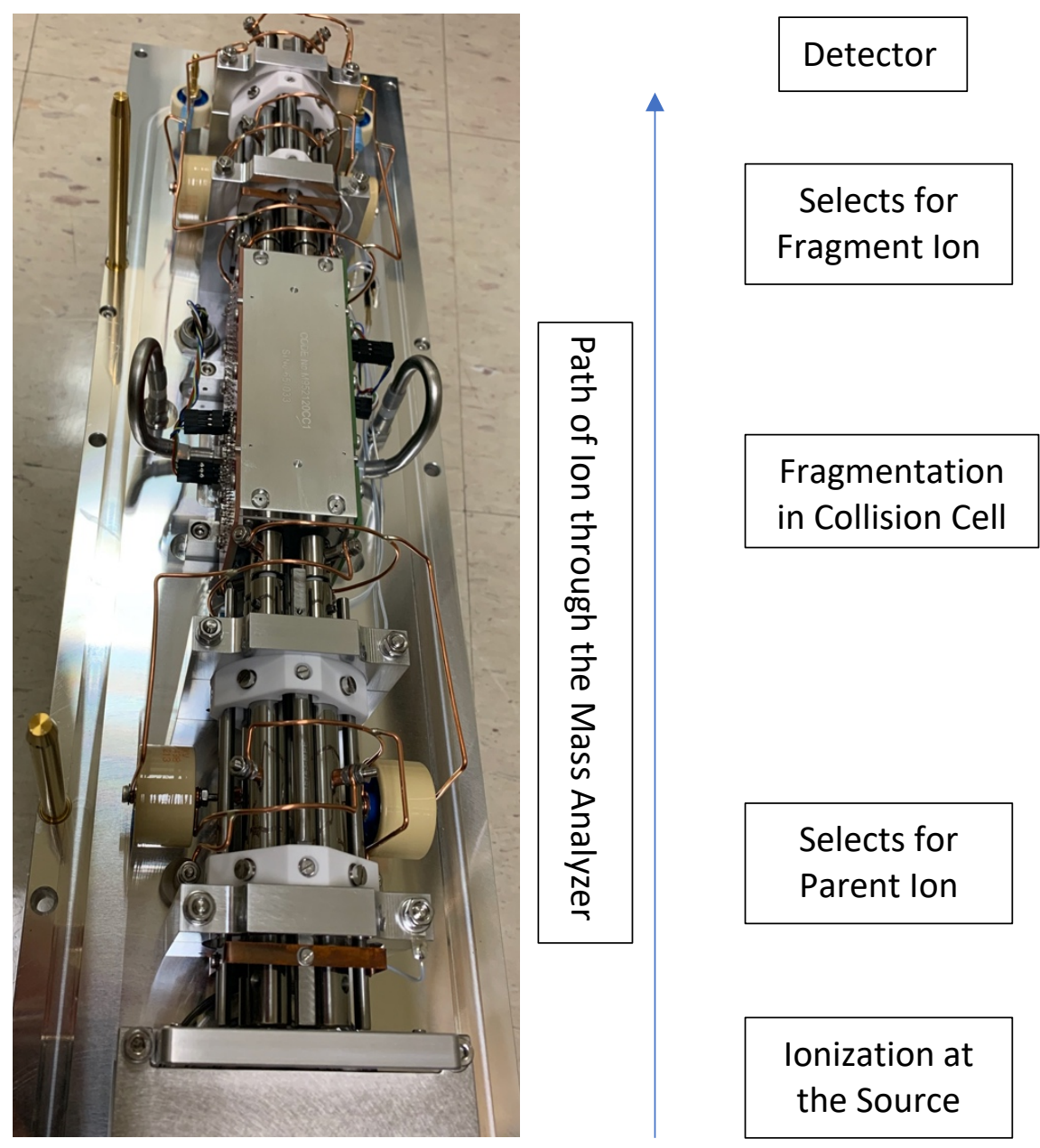

Figure 15. QqQ fragmentation schema. Photo of a QqQ from a Waters Acquity TQD, with sections labeled on the right.

Once stable transitions were found for each analyte, the optimal collision energy and cone voltage were recorded by manually increasing each parameter to observe a signal maximum for the fragment. Once the optimal cone voltage and collision energies were identified for each fragment, a preliminary MS method was created that included the $\mathrm{m} / \mathrm{z}$ 
for each transition pair, and the cone voltage and collision energy used for that transition. In addition to this MS method, the MS tune page settings were defined based on this experiment, including source temperature, the flow rate for desolvation gas, the capillary voltage of the ESI probe, ionization mode, and resolution settings. With the preliminary MS method created the next iteration of the method development process involved creating an LC method. The LC method needed to deliver the analyte to the MS in an appropriate MP mixture and desired selectivity to allow separation from potential interferants.

\subsubsection{Development of an LC Method for MMA, MMA-d3, and SA}

The process for the development of an LC method for the target analytes was a process of trial and error using literature approaches to inform study design. The fact that the analytes are all polar guided the design and allowed specific approaches to be ruled out quickly. The MS portion of the method needs to be built first to enable the compound's detection as it elutes from the column. If the experiment were attempted in reverse fashion, there would be no way to judge what chromatographic parameters made positive impacts on the MS signal, as the detector would not know what to measure.

With the MS parameters set and the MS method built, the LC optimization process began with MP selection. In this experiment, the stationary phases being tested include multiple column chemistry that span separation modes distinctly different, from RP to ANP and HILIC.

Initial experiments involved defining a preliminary gradient, experimenting with MP, and other chromatographic variables. Specifically, substituting various MP combinations 
in the $\mathrm{A}$ and $\mathrm{B}$ portions of the gradient, optimizing flow rate, and attempting different concentrations of MP additives were all initial experiments. Different column temperatures, the gradient slope and re-equilibration time were all varied to achieve reproducible chromatography. The variables observed to choose the ideal LC parameters included improvements to peak shape, resolution, effect on retention time, and selectivity for the various analytes. As will be discussed further in the Results section, this trial and error approach took many iterations before a baseline chromatographic peak on each stationary phase was observed. The decision to favor a chromatographic mode or MP composition was based on the empirically observed response in the MS and the impact on the chromatographic variables previously discussed.

There was no implicit idea of a specific combination that should work and an attempt to force the results towards that preconceived notion. Instead, each iteration's results were considered, changes to the LC method were made, and successive iterations were performed with an unbiased empirical approach to method development that would let the data decide the ideal approach. It should be noted that the column temperatures, gradient profiles, MP composition, and flow rates were held constant and compared across all three columns before changes were made. This approach intended to determine a baseline chromatographic peak on each stationary phase and then determine if changes made to any one of the tested variables had a positive or negative effect on the chromatographic peak and MS response.

Due to the number of variables being tested, the path to success was not linear. A result of the various chromatographic modes that were attempted caused numerous 
failures before success was attained. The various attempts will be discussed in the Results section, and some lessons learned that would inform subsequent method development attempts. It should be mentioned that the optimization of LC parameters is an iterative process. While early iterations have provided valuable information, there is still room for much improvement. This portion of the study will continue, as described in the Future Work section of this paper.

\subsubsection{Comparing Retention Modes of ANP and HILIC}

This study's primary purpose is to produce a viable clinical method for the quantitative measurement of MMA. This study's secondary purpose is to observe the differences in retention mechanism between ANP and HILIC separation modes. Two column chemistries were exposed to the same LC conditions for these experiments and the resulting RT trends observed. The column chemistries tested were $\mathrm{DH}$, the representative for ANP, and Amide BEH as the representative for HILIC. To further understand the difference in retention mechanism, a buffer salt experiment was undertaken. The first experiment involved varying the salt concentration in the MP from $10-100 \mathrm{mM}$ ammonium acetate and observing the effects on retention time across two stationary phases. All other LC variables were held constant. The initial experiments described above enabled observation of a baseline peak for each stationary phase, with mostly reproducible retention time. By altering the experimental variable, the salt concentration in the MP, the effect on retention time could be measured and correlated with an ANP or HILIC retention mechanism. 
The theory of ANP-LC would suggest that lower salt concentrations would result in longer retention times, whereas the HILIC retention mechanism displays the opposite trend. Therefore, while both ANP and HILIC can retain polar molecules, ANP does so most effectively at low salt concentrations, while HILIC is most retentive at high salt concentrations. The purpose of varying salt concentration in the MP on the DH column is to see if the data can verify this theoretical principle. 


\section{RESULTS AND DISCUSSION}

\subsection{Development of an MS/MS Method for MMA, MMA-d3, and $S A^{*}$}

The infusion experiments were successfully generated a preliminary MS/MS method, but it took several iterations and some troubleshooting to arrive at this endpoint. Progress depended on overcoming two significant challenges, including contamination of the infusion inlet with sodium adducts and low-end mass accuracy in the ESI negative mode. Besides, approaches using the software feature Intellistart, part of the MassLynx program, were inconsistent and needed to be verified both in the literature and through manual tuning experiments. However, many of these issues were not immediately apparent and required countless repeats and deliberation before solving one issue only to move onto the next. Despite these challenges, eventually, all were overcome, and a successful MS/MS method was developed utilizing a combination of Intellistart and manual tuning while utilizing a combined infusion setting. Before describing the process and various attempts to troubleshoot these issues, the final MS/MS method and tune settings can be seen in Tables 8 and 9 .

Table 8. ESI Negative Mode - MS/MS Preliminary Method

\begin{tabular}{|l|l|l|l|l|l|}
\hline $\begin{array}{c}\text { Compound } \\
\text { Name }\end{array}$ & Parent $(\mathrm{m} / \mathrm{z})$ & $\begin{array}{c}\text { Daughter } \\
(\mathrm{m} / \mathrm{z})\end{array}$ & Dwell (s) & Cone $(\mathrm{V})$ & $\begin{array}{c}\text { Collision } \\
(\mathrm{V})\end{array}$ \\
\hline MMA & 117 & 55 & 0.050 & 17 & 17 \\
\hline MMA & 117 & 73 & 0.050 & 17 & 8 \\
\hline MMA-d3 & 120 & 58 & 0.050 & 17 & 9 \\
\hline MMA-d3 & 120 & 76 & 0.050 & 17 & 9 \\
\hline SA* & 145 & 83 & 0.050 & 30 & 17 \\
\hline SA* & 145 & 101 & 0.050 & 30 & 14 \\
\hline
\end{tabular}


Table 9. MS/MS Tune Parameters

\begin{tabular}{|l|c|l|c|}
\hline \multicolumn{2}{|c|}{ Source (ES-) } & \multicolumn{2}{c|}{ Analyzer } \\
\hline Capillary (kV) & 2.80 & LM 1 Resolution & 10.54 \\
\hline Cone (V) & 17.00 & HM 1 Resolution & 15.00 \\
\hline Extractor (V) & 3.00 & Ion Energy 1 & 0.33 \\
\hline RF (V) & 0.10 & MSMS Mode & 20.00 \\
\hline Source Temp. $\left({ }^{\circ} \mathrm{C}\right)$ & & Collision Energy & 11.31 \\
\hline Desolvation Temp. $\left({ }^{\circ} \mathrm{C}\right)$ & 250 & HM 2 Resolution & 15.00 \\
\hline Desolvation Gas Flow (L/Hr) & 500 & Ion Energy 2 & 1.84 \\
\hline Collision Gas Flow (mL/Min) & 0.10 & Gain & 1.00 \\
\hline
\end{tabular}

\subsubsection{Contamination of the Infusion Inlet}

The Waters Acquity TQD used in this experiment is typically used for a clinical assay set up to detect compounds around one kilodalton in mass using ESI positive ionization mode. Historically, the MS/MS portion of the instrument was calibrated for mass accuracy annually with NaCsI solution, which has a series of monoisotopic peaks through the entire mass range and is well suited as a calibrant for ESI positive mode, especially in the mid to high mass range. Waters Corporation uses this calibrant as their standard tuning solution to calibrate LC-MS/MS instruments. ${ }^{51}$ A typical calibration of the mass accuracy is performed by loading a tuning solution onto the front inlet of the MS/MS, drawn up into an infusion syringe, and infused directly into the MS (Figure 16). 


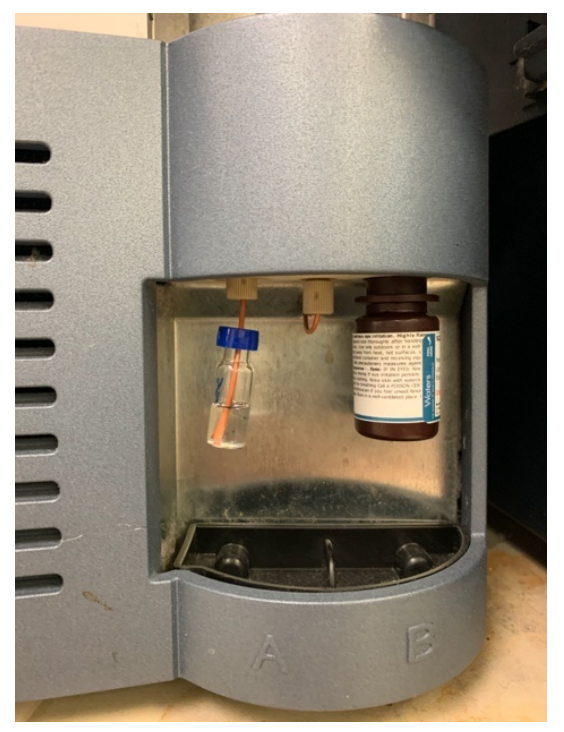

Figure 16. Front inlet infusion set-up for this instrument. Photo of a modified Infusion inlet on the Waters Acquity TQD instrument used.

The proper cleaning procedure for the inlet after using a solution like this would involve a 50:50 MeOH: $\mathrm{H}_{2} \mathrm{O}$ mixture. This mixture should be run through a purge cycle, in which the infusion syringe draws up several volumes of solution and then delivers it to waste. However, the instrument used in this study was not typically used for method development, nor was the front inlet touched for any reason. It should also be noted that the front inlet had been rebuilt and is not the typical stainless-steel reservoir tubing on both sides, but one side has plastic tubing, as can be seen in Figure 16.

Leaving a salt solution such as NaCsI in a reservoir that was in contact with the front inlet led to contamination with $\mathrm{Na}^{+}$adducts. The contamination with sodium adducts caused issues with Intellistart, as the software works in such a way that it locates the most abundant signal, and in the case of a high amount of sodium adducts eluting this signal was often positive. The Intellistart program saw a high positive signal. While running its optimization script, it would choose ESI + mode as the preferred ionization mode and 
return different masses each time it was run. The results did not make sense, but this was not instantly recognized as a contamination issue. The initial theory was that it was a software issue, as several error messages appeared at different points of running the script. One error message observed repeatedly can be seen in Figure 17.

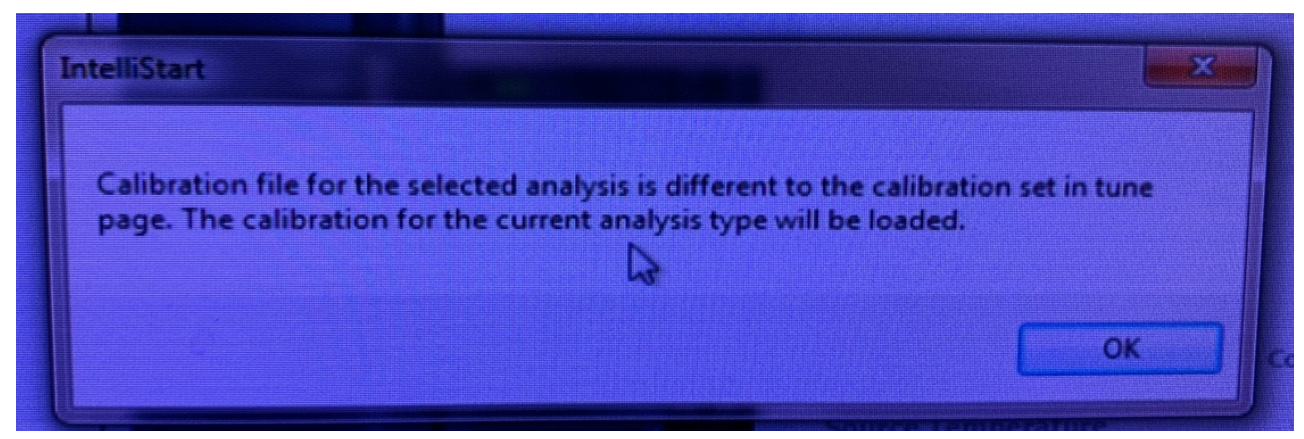

Figure 17. Intellistart recurring error message. Every time Intellistart was used during method development, this error would present.

While the error message seems straightforward, stating that there is an incompatibility between a tune file and a calibration file, it was impossible to rectify. The fact is that no combination of tune files and calibration files could make this error message go away. Even a tune file that is appropriately linked to a calibration set for the same MS resolution and double-checked before did not clear the error message. This inconsistency led to many hours reading forums, contacting Water's phone support, and e-mails with different service engineers until, it was ultimately discovered to be a common fault of this aging software version. According to a service engineer, this message will display to alert the operator that the resolution settings for the MS calibration and tune file must match, not that they do not match. While this is not what the wording seems to say, it was proven to be the case. 
Great care was taken to test this theory, selecting the proper MS calibration file, and making sure that the file was linked with the desired tune page. Using Intellistart set up, care was taken to specify the resolution desired, as "Unit Resolution," which needed to match the calibration file's resolution. Finally, utilizing the proper resolution settings paired to the proper calibration, which was linked with the matching tune page, yet the message persisted. Ultimately, a final attempt was made to generate a new tune page via Intellistart, based on the calibration, paired with the correct resolution settings for the MS/MS. This message persisted. Despite the confusion, this message caused it was not the reason for the confusing data being delivered.

Fortunately, software error was proven not to be the underlying issue affecting the observed inconsistencies in transitions found via Intellistart, however spurious results were still observed. Each time the program was run, a different target mass, and a different fragment ion were obtained and even a different ideal ionization mode for the same analyte. Once it was determined that the MassLynx Intellistart Software was working more or less as expected, despite some limitations based on a small number of bugs and the way the optimization script is written, the contamination issue was more clearly visible. If it is not software, it must be hardware, and based on the mode of use for front inlets and state of the equipment; it suggested contamination. To rectify this contamination issue, a 1:1 MeOH: $\mathrm{H}_{2} \mathrm{O}$ mixture was flushed through the front inlets on a purge cycle several times, with subsequent purge and refills of the compound to be measured. This approach did much more than methanol only purges to clear the inlet reservoirs. The cleaning's eventual outcome was that consistent results could be obtained 
from both reservoirs, but the results were not at the expected $\mathrm{m} / \mathrm{z}$ for the $[\mathrm{M}-\mathrm{H}]^{-}$ions in ESI negative mode.

\subsubsection{Low-End Mass Accuracy Issue in ESI Negative Mode}

Once the contamination issue and some of the software hurdles were overcome, manual tuning experiments were top priority. The analyte of interest was infused, as described above, at a concentration of $1 \mu \mathrm{g} / \mathrm{mL}$ in $\mathrm{MeOH}$. MMA's molecular weight is 118.09 Daltons when infused at $1 \mu \mathrm{g} / \mathrm{mL}$ in electrospray negative mode; it should yield a strong [M-H] $]^{-}$ion at $117 \mathrm{~m} / \mathrm{z}$. This experiment was attempted several times using Intellistart and again via manual infusion, but there was no signal present at $117 \mathrm{~m} / \mathrm{z}$. The same experiment was repeated for MMA-d3, the deuterated internal standard, at the same concentration of $1 \mu \mathrm{g} / \mathrm{mL}$ in $\mathrm{MeOH}$. Once again, both manual tuning and Intellistart were attempted for this compound, using the ESI negative mode. The choice to use ESI negative for these compounds was based on literature findings, preliminary infusion experiments, and carboxylic acid propensity to deprotonate in an ESI source. The expected [M-H] $]^{-}$ion for MMA-d3 should have yielded a strong signal at $120 \mathrm{~m} / \mathrm{z}$, yet once again, this was not the outcome (Figure 18).

\begin{tabular}{|l|l||l|l|l||l|l||l||}
\hline Compound & Formula/Mass & & $\begin{array}{c}\text { Parent } \\
\mathbf{m} / \mathbf{z}\end{array}$ & $\begin{array}{c}\text { Cone } \\
\text { Voltage }\end{array}$ & Daughters & $\begin{array}{c}\text { Collision } \\
\text { Energy }\end{array}$ & $\begin{array}{c}\text { Ion } \\
\text { Mode }\end{array}$ \\
\hline \hline MMA-D3 & 120.11 & 1 & 119.14 & 34 & 69.01 & 16 & ES- \\
\hline $\begin{array}{l}\text { MMA-D3- } \\
2\end{array}$ & 121.11 & 1 & 119.12 & 34 & 68.99 & 22 & ES- \\
\hline
\end{tabular}

Figure 18. Intellistart readout highlighting issue with mass accuracy. Data obtained from an Intellistart experiment showing a mass shift of 1 Dalton. 
By infusing and manually tuning for the compounds and attempting Intellistart, the unsatisfactory results could not be blamed on the Intellistart software. It should be noted that the correct formula mass in the chart above is the one given in the last row, 121. The only reason the formula/mass of 120 was given was to assess if the software expected a value with the $[\mathrm{M}-\mathrm{H}]^{-}$ion already calculated. However, it was found that this was not necessary, and in subsequent experiments, the correct formula/mass was entered. The expected parent $\mathrm{m} / \mathrm{z}$ in negative ion mode for MMA-d3 is $120 \mathrm{~m} / \mathrm{z}$, not the $119 \mathrm{~m} / \mathrm{z}$ shown above; this is due to the mechanism by which ESI ionizes. To aid in understanding a brief review of expected ionization patterns in ESI positive and negative modes will be discussed.

In positive ESI, a neutral ion can be protonated, forming an $[\mathrm{M}+\mathrm{H}]^{+}$ion, or it is possible another cation can be attached, resulting in an adduct ion formation, such as $[\mathrm{M}+\mathrm{Na}]^{+} .{ }^{37}$ In negative ESI, the converse occurs, with anion attachment or deprotonation commonly occurring. If a molecule has a carboxylic acid, which in the case of this study, all analytes studied have two carboxylic acids, a common loss is a proton, resulting in a $[\mathrm{M}-\mathrm{H}]^{-}$ion. It is also possible that an anion can be attached, such as $\left[\mathrm{M}+\mathrm{CH}_{3} \mathrm{COO}\right]^{-}$ion, which would be an adduct from an acetate ion. The typical mass spectra that ESI generates is dominated by molecular species, as it is a soft ionization technique. The exact process of ion formation in ESI is debated. However, it is believed to be based solely on an analyte's likelihood to ionize in solution once a voltage has been applied.

There is a known preference in ESI to overrepresent the most polar analytes in terms of response, due to the increased propensity to ionize. However, when coupled with LC, 
which often separates compounds based on polarity, this issue is less pronounced as the peaks eluting off the column will be delivered one at a time to the source reducing the in-source competition described above. In summary, the expected ion generated in ESI negative mode for a compound with a carboxylic acid, such as MMA, is a $[\mathrm{M}-\mathrm{H}]^{-}$ion, given the nominal molecular weight of 118 , is an $[\mathrm{M}-\mathrm{H}]^{-}$ion with $117 \mathrm{~m} / \mathrm{z}$. The expected ions for the other analytes in ESI negative mode are also [M-H]' ions, due to the carboxylic acids present, with ions of $120 \mathrm{~m} / \mathrm{z}$ for MMA-d3 and $145 \mathrm{~m} / \mathrm{z}$ for SA*.

The expected masses described above were not the observed masses in any infusion experiments, whether using Intellistart or manually tuning. The initial issue of sodium adduct contamination skewing Intellistart results to ESI positive mode, coupled with the software glitches observed using Intellistart, caused much repeating and rethinking of these initial MS/MS experiments. Comparing the expected theoretical results with literature findings, it was apparent there was an issue with mass accuracy. ${ }^{26}$

The real issue was more subtle and took the investigation of the instrument's historical use and previous preventative maintenance service logs from the manufacturer. Upon reviewing these logs, it was discovered that since the instrument was typically used in ESI positive mode, all historic mass calibrations had been performed in ESI positive mode exclusively. The calibration of mass accuracy with NaCsI in ESI positive mode was sufficient for current instrument applications. However, it did not provide adequate resolution for the low mass range in ESI negative mode. The infusion of a phosphoric acid solution was used to check the mass accuracy. An eventual recalibration of the mass scale in ESI negative mode with phosphoric acid was performed to correct the 
inaccuracy. The phosphoric acid calibration solution is an ideal tuning and calibration solution for the low mass range in ESI negative mode, with a monoisotopic ion at $99 \mathrm{~m} / \mathrm{z}$. Once the instrument had been re-calibrated in ESI negative mode with the phosphoric acid calibrant, the mass accuracy issue was corrected, and the tuning experiments progressed. The calibration with phosphoric acid in negative mode can be seen in Figure 19.

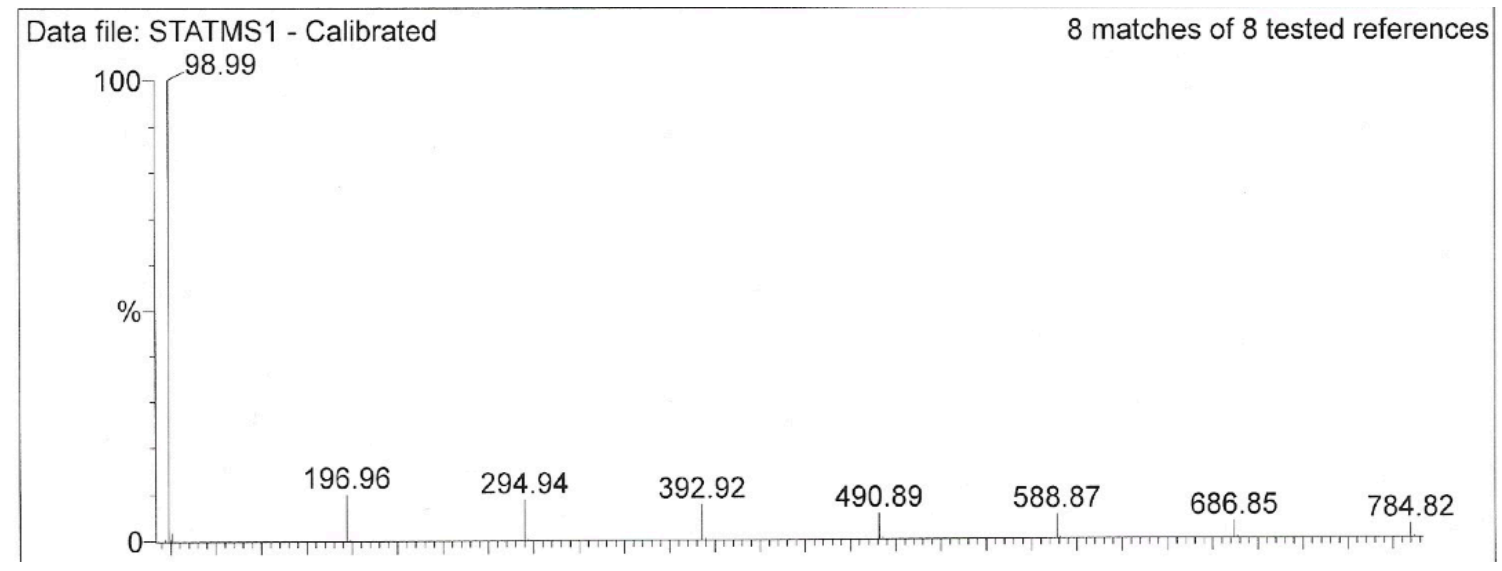

Figure 19. Calibration with phosphoric acid in ESI negative mode. A series of monoisotopic peaks in a phosphoric acid solution is used to set the mass accuracy in ESI negative mode.

After successfully calibrating the MS/MS's mass accuracy, the infusion experiments generated the expected $[\mathrm{M}-\mathrm{H}]^{-}$ions, which allowed Intellistart experiments supplemented with manual tuning to be performed. The correction of the underlying mass accuracy and contamination issues, combined with forcing Intellistart only to use the negative ESI mode, allowed useful data to be obtained from Intellistart.

\subsubsection{Path to a Successful MS/MS Method}

Intellistart was used as a starting point, but the transitions found were often incomplete, and subsequent manual tuning experiments were performed to supplement. 
An example of an Intellistart transition found, and one missed can be seen in MMA's case (Figure 20).

IntelliStart found the following compounds:

\begin{tabular}{|l|l|l|l||c||c||c||c||}
\hline Compound & Formula/Mass & & $\begin{array}{c}\text { Parent } \\
\mathbf{m} / \mathbf{z}\end{array}$ & $\begin{array}{c}\text { Cone } \\
\text { Voltage }\end{array}$ & Daughters & $\begin{array}{c}\text { Collision } \\
\text { Energy }\end{array}$ & $\begin{array}{c}\text { Ion } \\
\text { Mode }\end{array}$ \\
\hline \hline MMA & 118 & 1 & 116.90 & $* 18$ & 54.98 & 21 & ES- \\
\hline
\end{tabular}

Figure 20. MMA Intellistart readout after MS/MS calibration. Incomplete data obtained from Intellistart; however, mass accuracy has been corrected.

The Intellistart software did return the correct parent $\mathrm{m} / \mathrm{z}$ after calibration of mass accuracy with phosphoric acid. However, it only found one transition. Upon further review of the report generated by this software program, it had obviously missed another viable transition, with a fragment at $73 \mathrm{~m} / \mathrm{z}$ (Figure 21 ). 


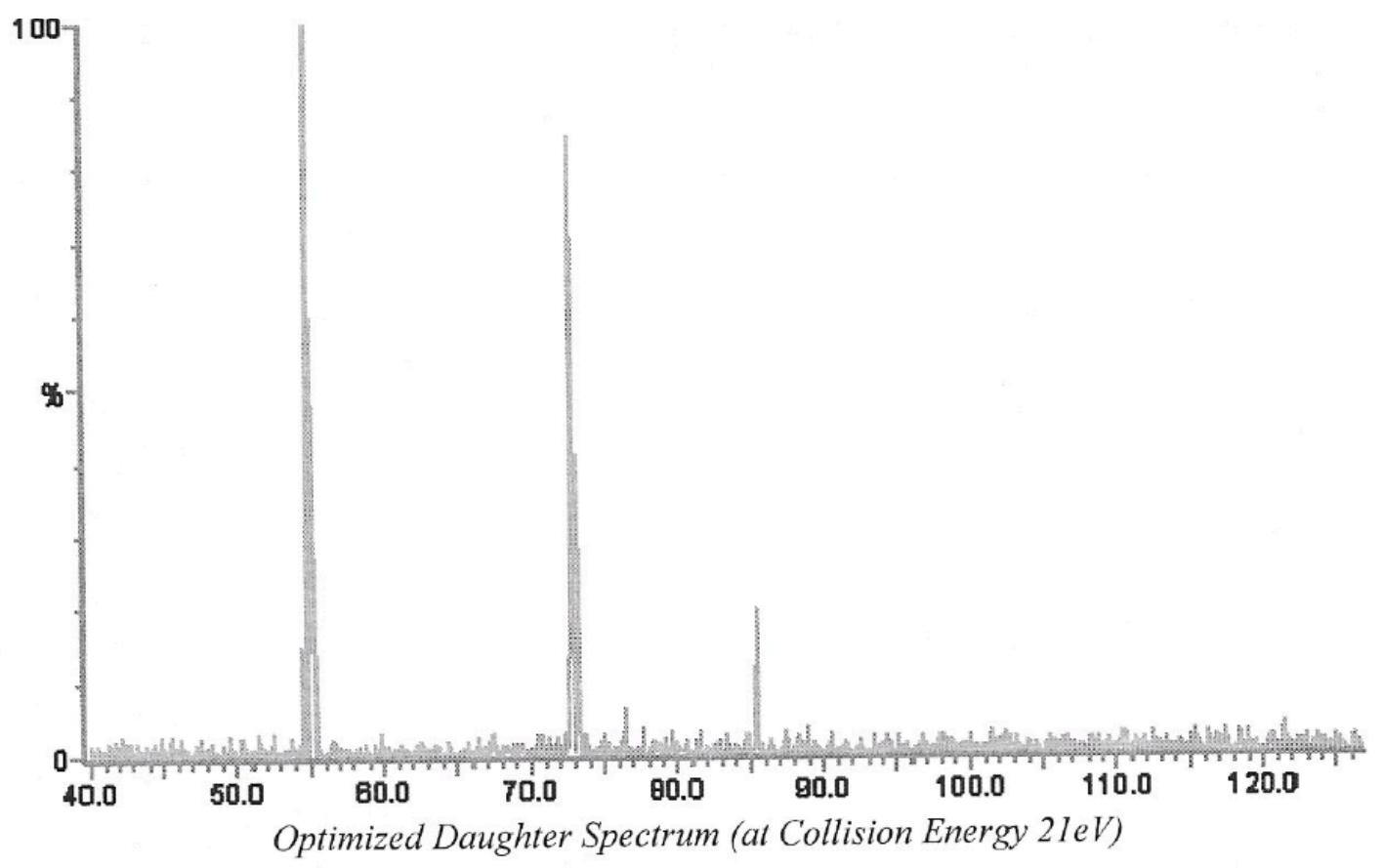

Only 1 stable transitions identified for MMA using the requested ion modes.

Figure 21. Intellistart report with only one stable transition. Data from an Intellistart report, states only one stable transition, while two are clearly visible and later verified.

While the Intellistart program returned a finding of only one stable transition, there is clearly a second fragment at $73 \mathrm{~m} / \mathrm{z}$, which was missed by this software (Figure 21). Manual tuning experiments were performed to investigate this second potential transition, where MMA was directly infused with the collision gas turned off, and MS1 was set to select for $117 \mathrm{~m} / \mathrm{z}$. Once MS1 had a stable signal at $117 \mathrm{~m} / \mathrm{z}$, all tuning parameters were adjusted to optimize signal strength (Figure 22). 


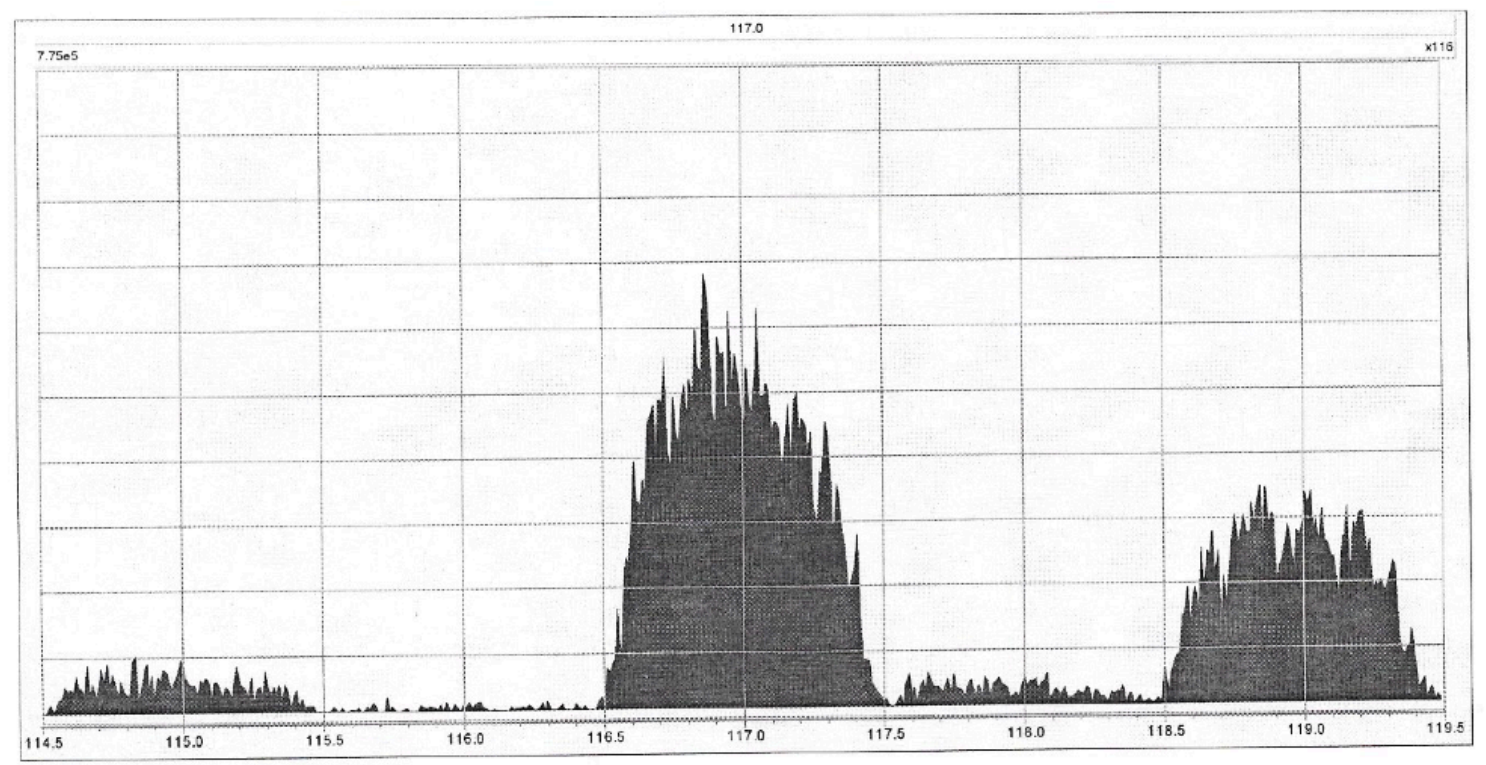

Figure 22. Manual tuning and optimization of $[\mathrm{M}-\mathrm{H}]^{-}$ion for MMA. Data obtained from a manual tuning experiment while infusing MMA in ESI negative mode.

The manual tuning optimization experiments were accomplished by infusing the compound at $1 \mu \mathrm{g} / \mathrm{mL}$ concentrations in $\mathrm{MeOH}$ at a flow rate of $20 \mu \mathrm{L} / \mathrm{min}$, with a combined infusion setting that delivered a constant flow of the MP at $800 \mu \mathrm{L} / \mathrm{min}$. The MP composition for this experiment was eighty-five percent acetonitrile, fifteen percent LC-MS/MS grade water with $100 \mathrm{mM}$ ammonium acetate and one percent formic acid, as specified in the methods, and modified from a successful approach in the literature. ${ }^{26}$ The cone was cleaned daily during infusion experiments due to the high salt concentration of the MP and constant infusion at a high flow rate. The results of the successful tuning experiments can be seen in Table 9 in section 3.1.1.

Once the ideal MS acquisition parameters were established for the parent ion and the collision gas was turned on. The collision energy was set to zero and slowly ramped upward, and the effect on the fragment ion was observed. In this manual tuning 
experiment, the first quadrupole, MS1, was set to select for only the $117 \mathrm{~m} / \mathrm{z}$ fragment, and a daughter scan was performed with a small span centered on the expected fragment ion at $73 \mathrm{~m} / \mathrm{z}$ (Figure 23 ).

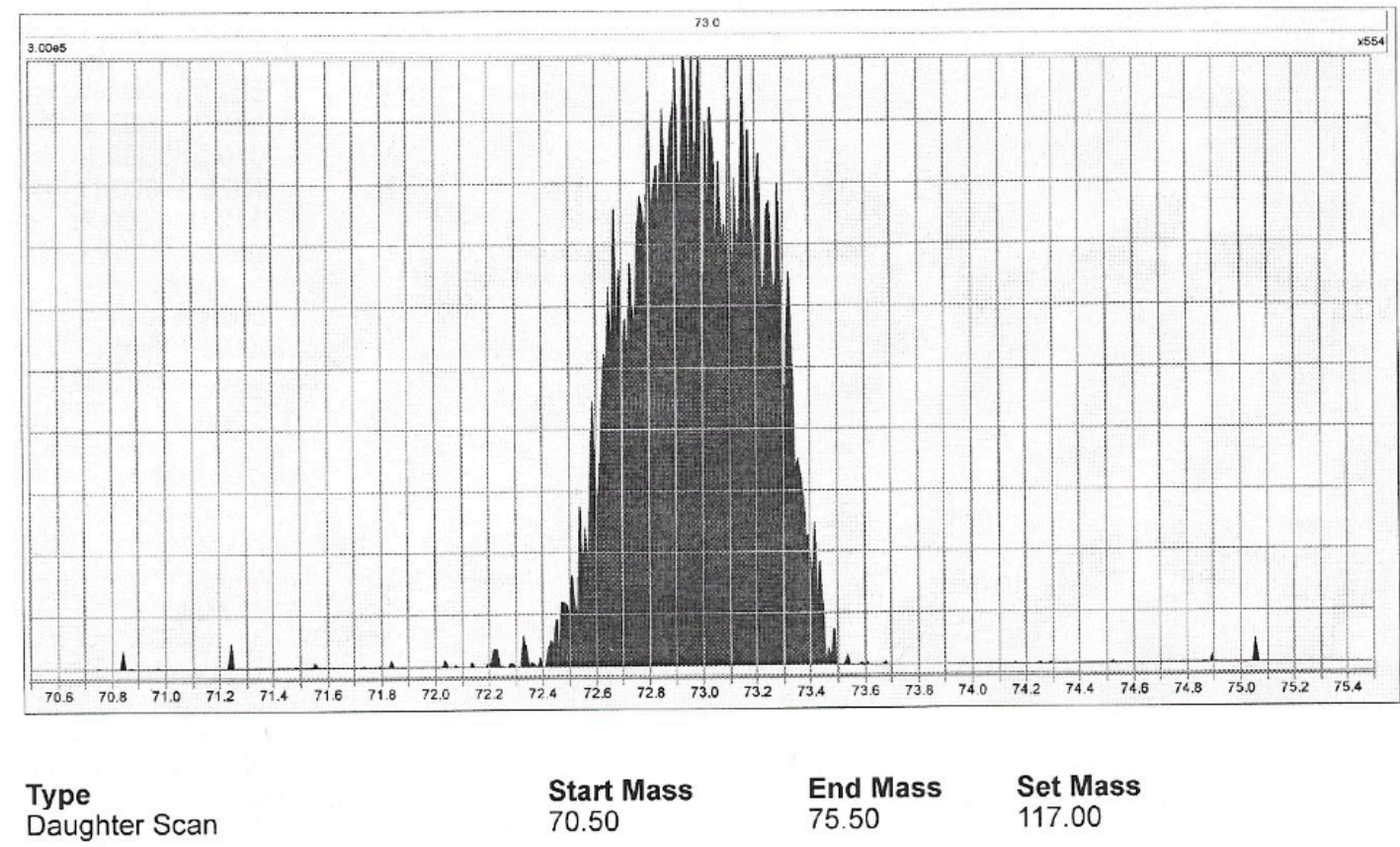

Figure 23. Manual tuning of fragment at $73 \mathrm{~m} / \mathrm{z}$. Data obtained from a direct infusion experiment in ESI - mode with collision gas turned on.

A daughter scan is where MS1 is restricted to a certain mass, but MS2 is open to record all fragment masses. As the collision energy is ramped from zero upwards, the abundance of the parent ion decreases, and the fragment abundance increases, but only to a certain point. As the collision energy is ramped higher, eventually, the abundance of the desired fragment decreases. The most abundant fragment would often shift to a lower $\mathrm{m} / \mathrm{z}$ due to the increased fragmentation. The goal of manual tuning is to find the optima in the curve and utilize that setting to then ramp and optimize the other settings. This process is an iterative one and can produce odd results if only approached in linearly. For 
example, if the collision energy were ramped up to $100 \mathrm{eV}$, the maximum on the scale, the only fragments visible are low mass fragments. Any further optimization done to other parameters would not change this fact.

A successful approach is to establish a maximum, utilize that setting, then after establishing other settings, come back and re-ramp the initial parameter to make sure subsequent choices or prior settings on the first iteration do not bias the results. This iterative process affords a better opportunity of finding a global maximum rather than a local maximum. In addition to collision energy, the manual tuning process was performed to optimize cone voltage. The findings from these experiments were used to determine the preliminary MS/MS method shown in Table 8 in section 3.1.1. The mass calibration setting chosen to link with the tune page was "Unit Resolution," which offered increased signal strength while maintaining good selectivity.

In summary, the MS/MS method development of this process proved to be much more involved than previously anticipated. The number of hurdles that needed to be overcome and the disparity of the early results obtained resulted in several prime opportunities for troubleshooting. Unfortunately, the inability to establish an MS/MS method with ease led to significant delays in LC method development. The MS/MS method is a requirement before any of the $\mathrm{LC}$ work could begin because a detector is needed to measure analytes eluting from the column. Without a properly functioning detector, there is no way to record the peaks or observe chromatographic differences between the stationary phases. In the end, the solutions arrived at seem quite obvious, but during the process, this was not the case. Ultimately, the lessons learned are 
fundamental laboratory concepts, measurements are only as good as the condition of the instrument; cleanliness, regular maintenance, and a recent mass calibration are all necessities before beginning any method development process.

\subsection{Development of an LC Method for MMA, MMA-d3, and SA*}

The LC portion of the LC-MS/MS method development was challenging for many reasons, but several insights can be gleaned from the preliminary experiments performed. Some of the challenges included working with three stationary phases, all representing a distinct chromatographic mode, RP, ANP, and HILIC, respectively. Inexperience in method development, combined with atypical solvent selection and high salt concentrations, led to high back pressures, which caused a few instrument issues including column leaks, many experiences cleaning the skimmer cone, and the ESI capillary probe rebuild. Other than the troubleshooting experience, the significant insights gained can be summarized in three sections: (1) preliminary RT and peak shape across the various columns, (2) effects of priming and trends with repeat injections, and (3) selectivity and resolution differences across the three columns tested. Several other topics will be touched upon, including points sampled in a given peak, peak symmetry, and carryover. Ultimately, it will be shown that while all three stationary phases can separate the analytes of interest, reproducibility and peak shape vary widely. While much work remains for a polished method, DH appears to be a good candidate for further optimization experiments based on resolution and peak shape. 


\subsubsection{Preliminary RT and Peak Shape}

The initial experiments were performed by injecting each compound one at a time at a concentration of 1 microgram $/ \mathrm{mL}$ on each column using the preliminary gradient shown in Table 10.

Table 10. Preliminary Gradient

\begin{tabular}{|c|c|c|c|c|c|}
\hline & Time (min) & Flow $(\mathrm{mL} / \mathrm{min})$ & $\% \mathrm{~A}$ & $\% \mathrm{~B}$ & Curve* $^{*}$ \\
\hline 1 & Initial & 0.750 & 100 & 0 & Initial \\
\hline 2 & 0.10 & 0.750 & 100 & 0 & 1 \\
\hline 3 & 2.00 & 0.750 & 50 & 50 & 6 \\
\hline 4 & 2.25 & 0.750 & 0 & 100 & 1 \\
\hline 5 & 3.00 & 0.750 & 100 & 0 & 6 \\
\hline 6 & 4.00 & 0.750 & 100 & 0 & 6 \\
\hline
\end{tabular}

${ }^{*}$ Curve values: $1=$ Pre-Step, $6=$ Linear Gradient

The other parameters used were a column temperature of $40^{\circ} \mathrm{C}$, a 10-microliter injection using a 50-microliter sample-loop, and the sample manager temperature was held at $10^{\circ} \mathrm{C}$. The MPs used for these successful initial experiments were, ethyl acetate for MP A, and a mixture of 80:20 acetonitrile:water with $100 \mathrm{mM}$ ammonium acetate and $1.0 \%$ formic acid. The decision to use ethyl acetate as a loading or weak MP was due to its miscibility with water and acetonitrile, weak polarity and success in related literature. ${ }^{26}$ The polarity scale for the MPs used can be ranked in terms of relative polarity as, ethyl acetate 0.228 , acetonitrile 0.460 and water $1.000 .^{52}$ Attempts were made using typical RP solvents. However, the water-based loading MP's polarity caused elution within the void volume on $\mathrm{C} 18$, as would be expected.

Trials using a more traditional RP-LC based MP mixture with a water-based MP (A) and an acetonitrile-based MP (B) with the same preliminary gradient described above in Table 10. While this MP combination resulted in decreased back pressure and more 
reproducible retention times (Figure 24), the peak shape was quite poor across the five injections, and the analytes co-eluted (Figure 25).
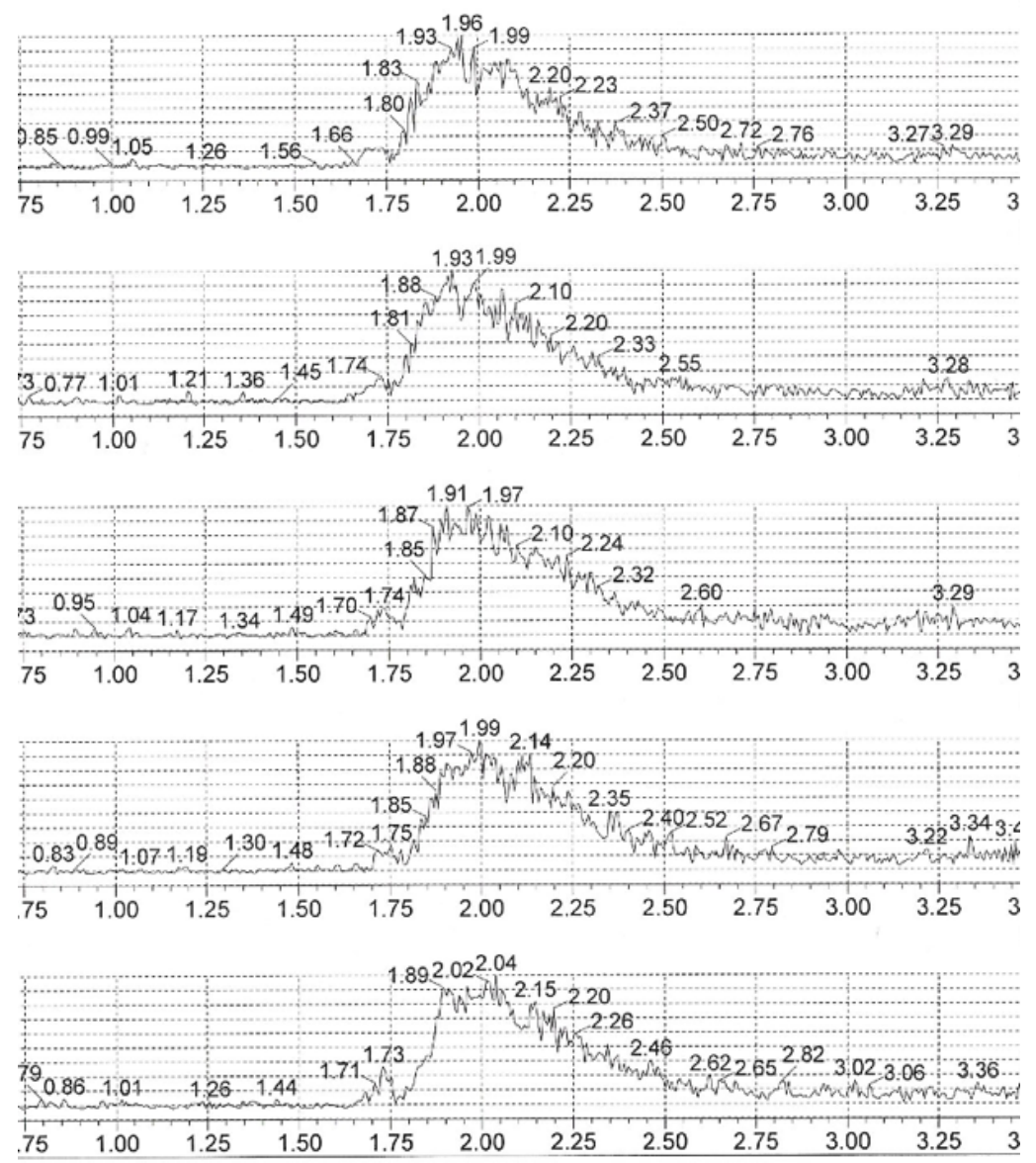

Figure 24. Stable RT on DH - 5 Injections. Repeat injections with RP MP mixture. Total Ion Chromatograms (TICs) displayed with time in minutes on the x-axis. MMA, MMA-d3, and SA* all present. 

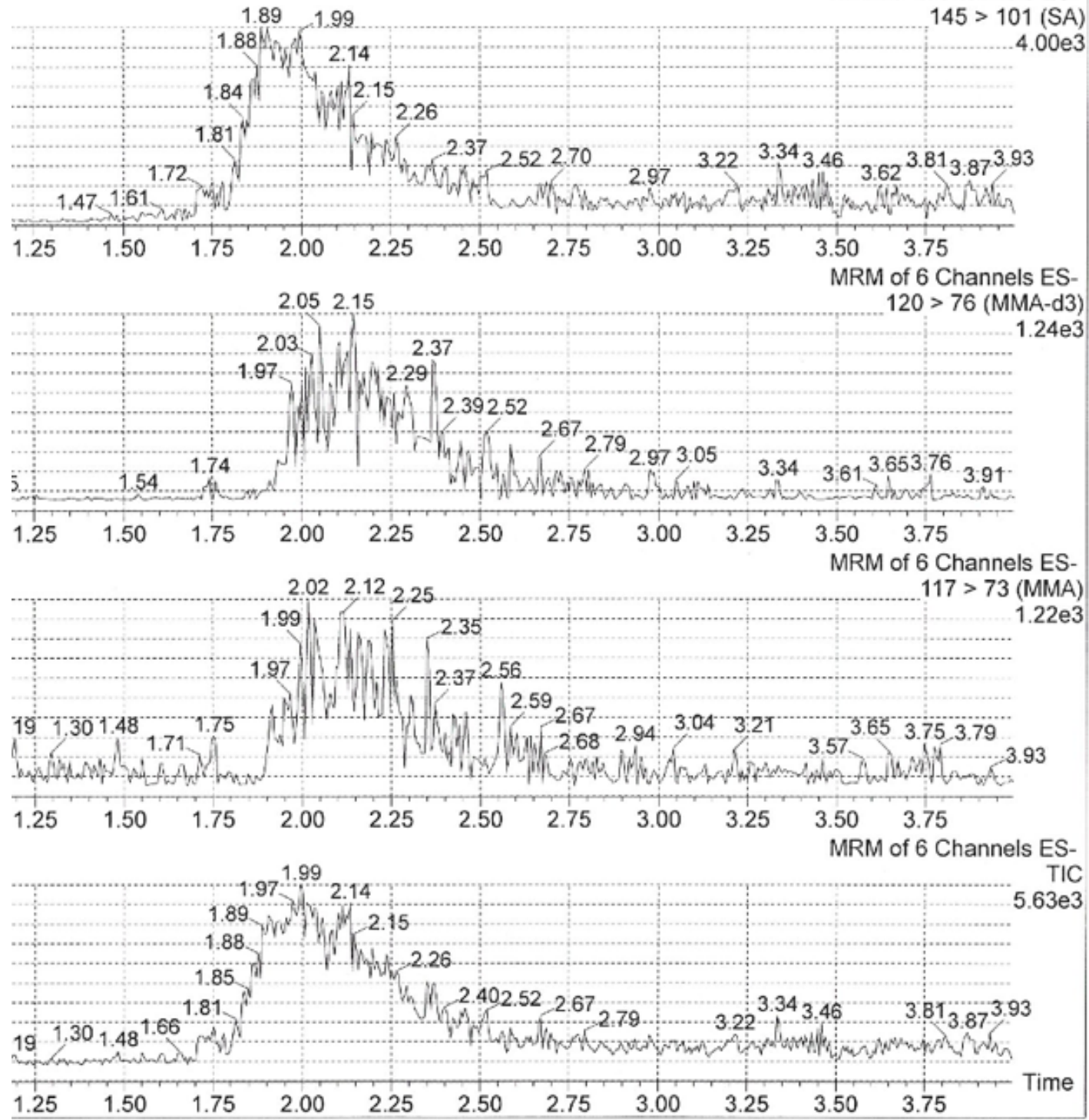

Figure 25. DH - one Injection by transition. One of the TIC's shown above in Figure 24 is displayed by $\mathrm{m} / \mathrm{z}$ transition. Note analyte co-elution and poor peak shape. The time on the $\mathrm{x}$-axis is listed in minutes.

The peak shape, early elution, and co-elution problems for this RP-LC MP mixture on $\mathrm{DH}$ highlighted some issues using water as a loading MP for analytes of this polarity (Figure 24). In addition to the issue with co-elution of SA* and MMA on DH when water-based weak MP was used, there were significant issues with back pressure while 
attempting to equilibrate the Waters BEH-Amide column. In fact, the Waters BEHAmide column could not accommodate $100 \%$ water at a flow rate of $0.750 \mathrm{~mL} / \mathrm{min}$ without back pressures approaching 15,000 PSI, so the method would have to be adapted even to get preliminary injections accomplished. The BEH-Amide column is twice the length it is expected that a slightly higher back pressure is warranted, however, attempts were not made to extend the run time and decrease the flow rate as it would not serve a very fair method comparison.

Another MP mixture that was attempted to separate all three analytes on the DH column was a more traditional HILIC/ANP water and acetonitrile combination. As with the injections in Figures 24 and 25, the injections displayed in Figure 26 were performed with the same preliminary gradient displayed in Table 10. 


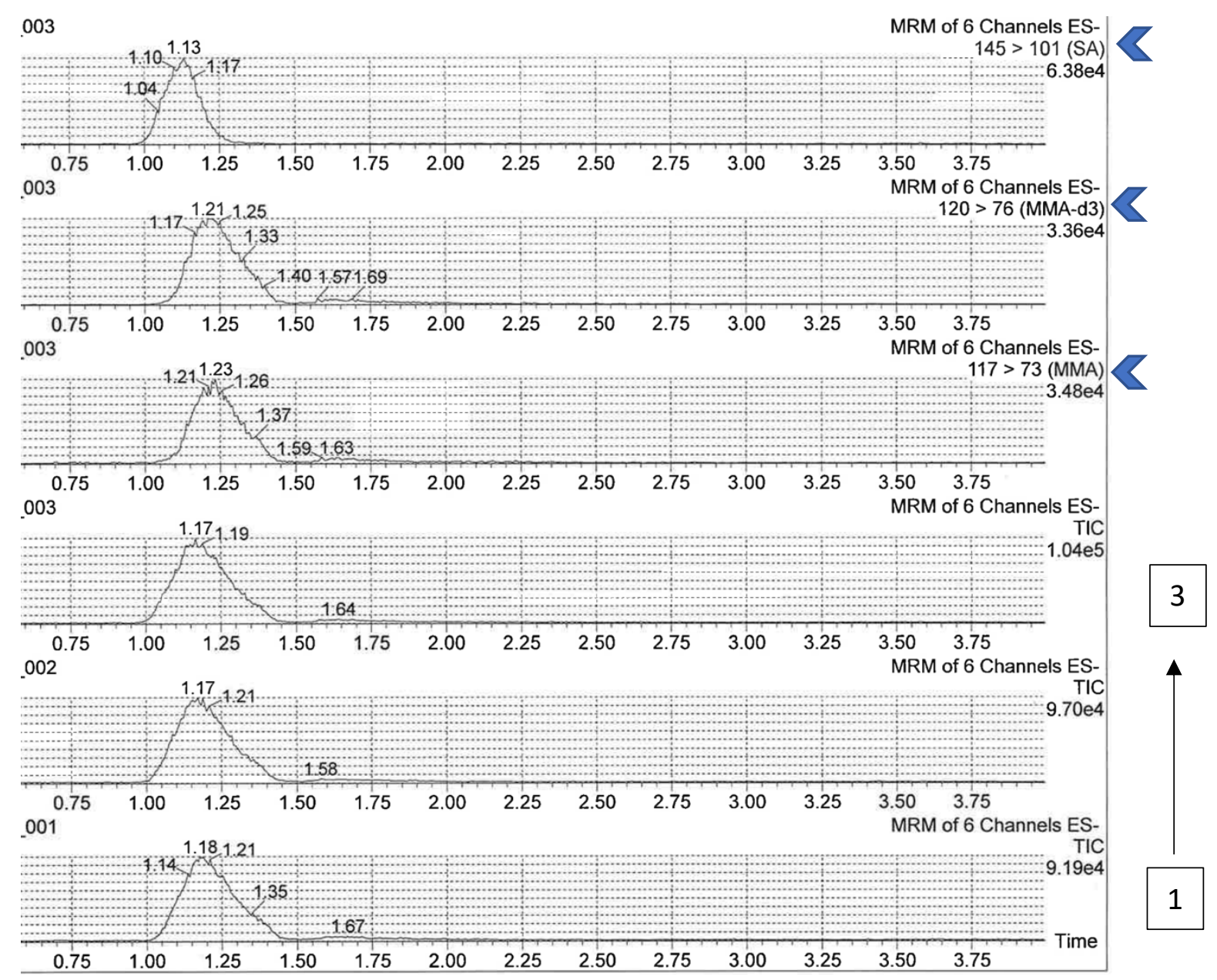

Figure 26. Classical HILIC/ANP MP mixture on DH. 3 injections plus transitions. The MP composition for the data shown is 80:20 acetonitrile:water loading MP, with a 50:50 acetonitrile:water, strong MP. In both MP, the additive concentrations are held constant at $10 \mathrm{mM}$ ammonium acetate with $0.1 \%$ formic acid. The $\mathrm{x}$-axis shows time in minutes. 1-3 are TIC's representative of repeat injections. Arrows highlight compounds used.

The three-injection series using this typical HILIC/ANP MP mix demonstrated reproducible RT's as displayed in the bottom three panes of Figure 26. Relatedly the back pressures were significantly less with this mixture, and much less priming was required to achieve this stability. However, the analytes all co-eluted as can be seen in the top three panes of Figure 26, which are the individual $\mathrm{m} / \mathrm{z}$ transitions for the third injection shown in the series, TIC labeled 3. In addition to the analytes co-eluting, they 
eluted very early, even before the gradient began. These results suggested that a waterbased loading MP, even if only $20 \%$, would cause the compounds to elute early and together. In fact, the often used HILIC/ANP MP with 80:20 acetonitrile:water worked more like an elution MP for these compounds. Due to these challenges and the success in the literature using the non-traditional MP mixture, the decision to try ethyl acetate as a loading MP resulted in some promising chromatography.

Ethyl acetate, as a loading MP, instantly showed improvements in peak shape and separation of SA* and MMA. Despite the promise of ethyl acetate, there were miscibility issues, which will be discussed. The preliminary LC gradient and nontraditional MP conditions described above resulted in retention on all three columns, as shown in Figure 27. 

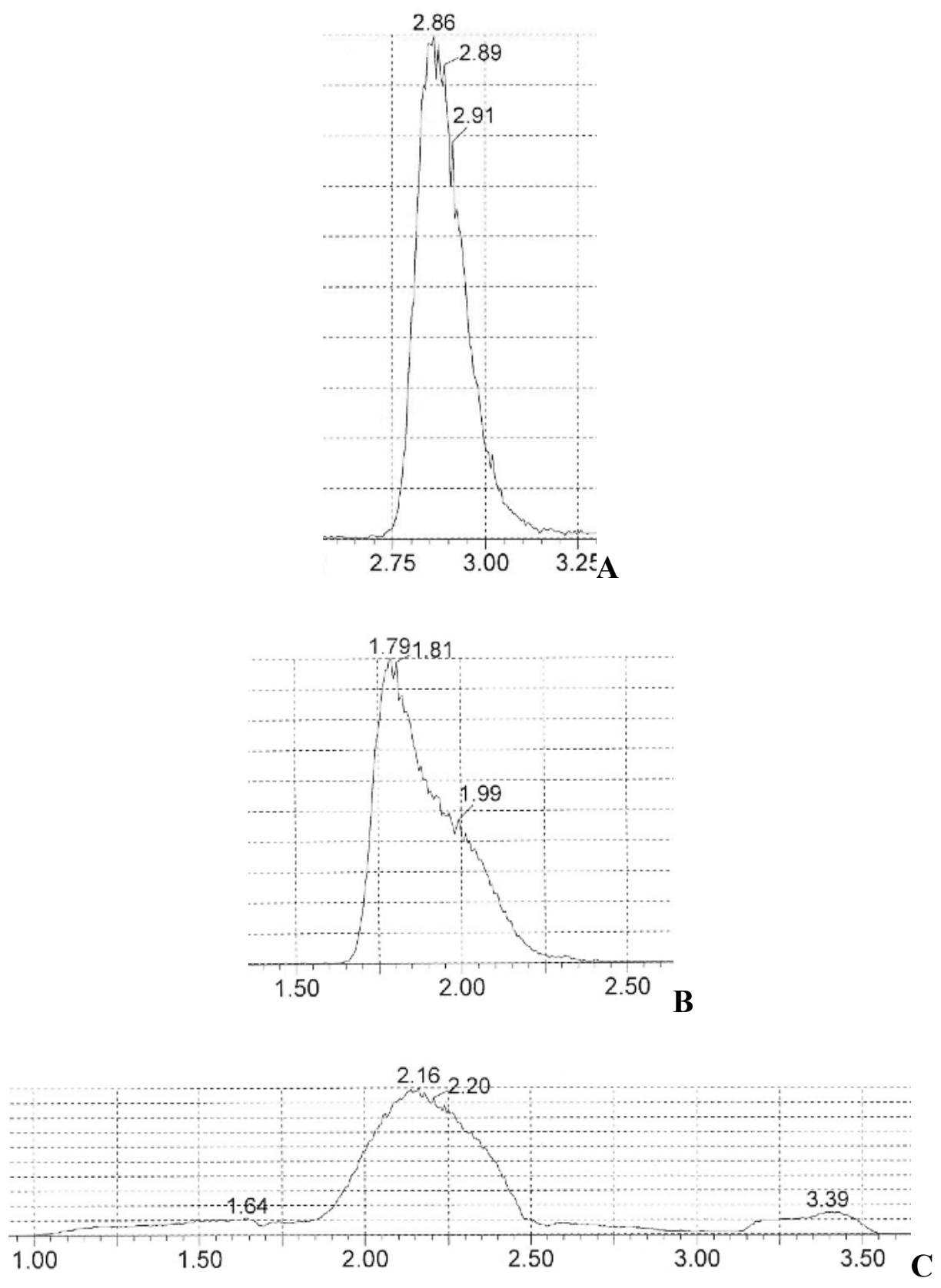

Figure 27. MMA-d3 preliminary injections. (A) DH (B) C18 (C) BEH. One compound (MMA-d3) injected across three columns, with the same gradient and MP mixture used. The $\mathrm{x}$-axis has the time listed in minutes.

The peak shapes above show distinct differences in width, skew, and absolute retention time (Figure 27). It should be noted that although the column chemistries 
represent distinct chromatographic modes, the same gradient, flow rate, and MP composition were used across all three for comparison. Though not visible in these graphs, the same concentration of analyte was injected, $1 \mu \mathrm{g} / \mathrm{mL}$, and relatively the same response was achieved at the detector, roughly $5 \mathrm{e} 4$. Using the dwell times listed in Table 8, the peak widths at half height and an MS delay of 5 milliseconds, the sampling points per peak are greater than 20 for all three peaks, so sufficient time is available to acquire useful MS/MS data for all six transitions.

The sharpest and most gaussian peak is obviously the one eluting from the DH column at 2.86 minutes, with a peak width of just under 8 seconds and almost no skew (Figure 27A). The peak eluting off of the $\mathrm{C} 18$ column was the least retained, with an RT of 1.79 minutes, a peak width of almost 10 seconds, and a clear shoulder on the right side about halfway down (Figure 27B). The peak eluting off the BEH amide column has many issues, both fronting, and tailing, and peak width of nearly 26 seconds (Figure 27C). These initial results were suggestive that $\mathrm{DH}$ was an excellent choice of stationary phase for a polar molecule like MMA. Of course, there was still much room for improvement in peak shape and response, but reproducibility had to be addressed first.

\subsubsection{Reproducibility - The Trends of RT and Peak Shape}

One of the most critical factors to reproducibility over a series of injections is the ability to regenerate the initial injection conditions. These initial conditions consist not only of solvent composition but also system back pressure. As read out by the binary solvent manager, system back pressure changes or deltas are commonly monitored to ensure the stability of fluidics before beginning injections. Other factors influencing 
reproducibility in these experiments include on-column carryover and instrument condition.

The first lesson learned in UHPLC-MS/MS method development with atypical MP combinations, compared to "typical" RP-LC MPs, is the importance of a good prime. The eventual priming protocol adopted to transition this multi-assay instrument to an atypical HILIC/ANP type mixture of mobile was a multi-step process. First, priming of the PEEK solvent lines for several minutes, running to waste, then priming the column with a mixture of water and methanol for several minutes and finally priming the column at initial conditions for at least thirty minutes. The reason for this degree of instrument set-up was the result of accidentally trying some injections on a column that was not yet adequately primed (Figure 28). 


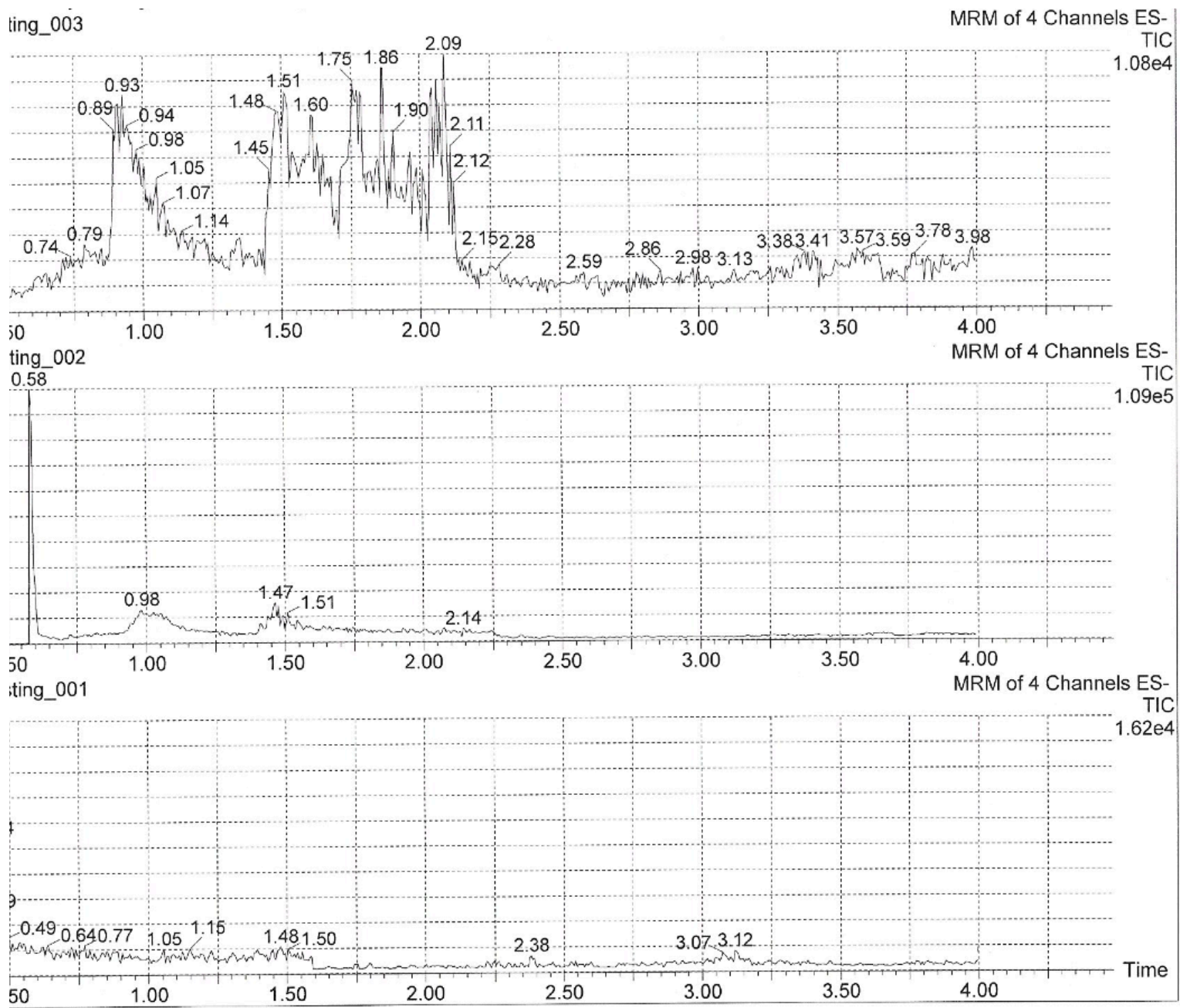

Figure 28. Three injections in a row without prime. The same compounds in three repeat injections, TICs displayed in all three panes. The x-axis is listed in minutes - no resemblance between the three injections due to unstable chromatography.

Changes in back pressure with a constant flow rate can result in non-laminar flow and chromatographic abnormalities. While most initial injections were performed after several iterations of priming as described above. On a few occasions, like the data from the C18 column in Figure 28, it was clear that an inadequate prime did not produce valuable data. 
The questions then became how much initial priming is necessary and how long an equilibration time is necessary to regenerate the low deltas in the binary solvent manager pressure readout to achieve reproducibility. Unfortunately, this is still a question that remains to be answered as reproducibility was a challenge throughout the project and remains a focus of future work. The reality was that some days the priming protocol above would result in deltas in the $<10$ PSI range. However, at other times, the instrument could prime for over an hour and not stabilize below a delta of 200 PSI. This inconsistency made some days tend toward troubleshooting rather than data collection; some of these tips will be discussed. Other than the instrument issues discussed subsequently, the miscibility of ethyl acetate, acetonitrile, water, and high salt concentrations proved challenging. The salt concentrations used in the experiments included $100 \mathrm{mM}$ ammonium acetate with $1 \%$ formic acid, infused at a relatively high flow rate on a small internal diameter column resulted in high back pressure. Experimental conditions resulted in back pressure approaching the instrument maximum 15,000 PSI. This considerable variation could be explained by a potential solubility issue with the high salt concentration and ethyl acetate, resulting in an on-column salting-out that could take several volumes of a more polar solvent to flush out.

Three instrument issues confounded reproducibility and led to a good deal of deliberation, but ultimately were fixed and still did not result in absolutely stable chromatography. One of the issues was potential turbulence at the column installation point, which involved re-seating the column with the appropriate hardware and determining there was no dead space. As several other individuals used this instrument, 
at one point, a turbulence check on the front end of a column revealed a missing ferrule, which created a conical dead space and mixing. The ferrule was easily re-installed and checked regularly after that. Instrument issue number two, a dirty skimmer cone, was caused by the use of high salt concentration, lengthy infusions, and the high flow rate with aqueous solvents (Figure 29).
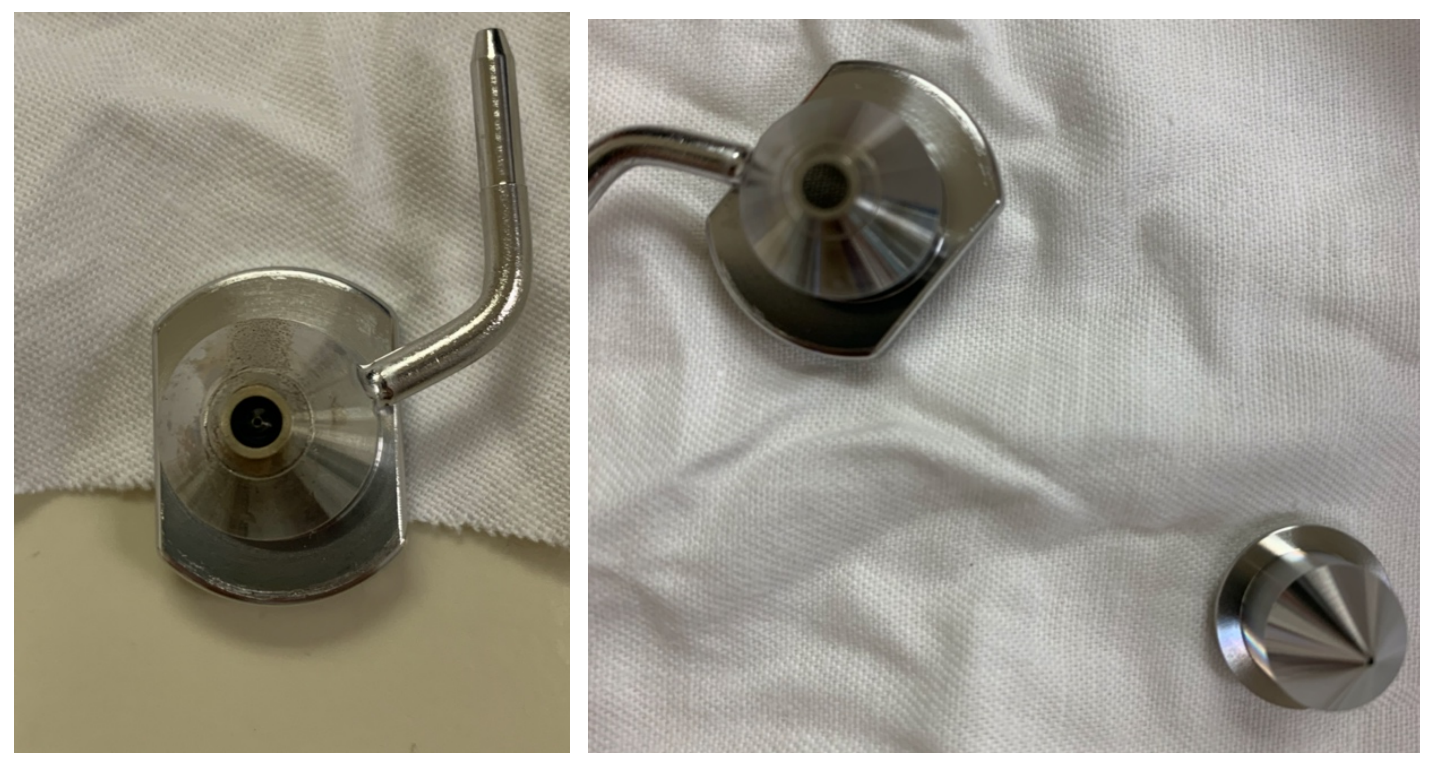

Figure 29. Dirty skimmer cone (left). Clean skimmer cone and cone (right). A consequence of the high salt concentration used in the MP mixture was constantly dirtying the skimmer cone.

At various times in method development, the response would drop, and background would increase, but regular cleaning of this skimmer cone would significantly ameliorate that issue. The eventual protocol adopted was to install a clean cone at the beginning of the day and clean the old one to re-install at the end of the day. Method development is an aggressive process from the instrument's perspective, and care must be taken to reduce the number of extraneous variables impacting results. The final instrument issue that 
resulted in some unstable MS/MS signals initially and a total instrument downtime subsequently was the ESI capillary probe being pushed out at a high flow rate.

How long the probe was loose is tough to say, but the eventual push that caused it to dislodge fully was a column prime at $2 \mathrm{~mL} / \mathrm{min}$ flow rate that should have gone to waste, but the divert valve was in the wrong position. This costly error caused the ESI capillary probe to extend deep into the source housing, and instead of spray, as electrospray is known for, there was dripping of liquid directly onto the cone shown in Figure 30.

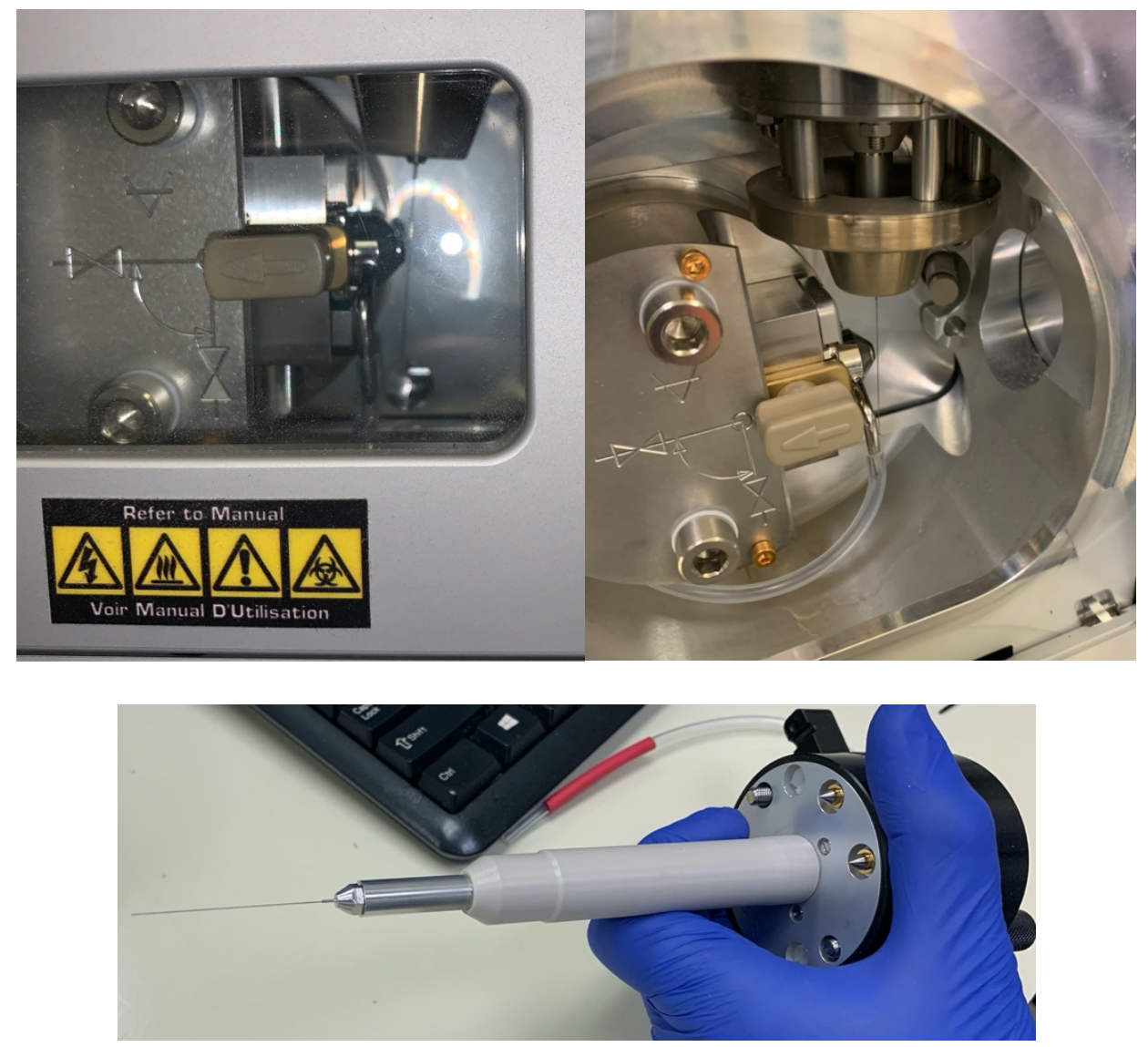

Figure 30. ESI capillary probe dislodged. The probe in its dislodged state (top) and the rebuild (bottom). 
The tip of the ESI capillary is supposed to protrude $0.5 \mathrm{~mm}$, but as shown in Figure 30, it was hanging well past the skimmer cone, and the liquid was running down it. The probe was rebuilt to specifications and re-installed with no lasting damage to the hardware, yet this caused some of the data acquired directly before the dislodge to be unusable as it showed a great deal of variation in response at the detector, likely due to the presence of liquid on the cone. While these instrument issues in hindsight may seem mundane, they result in lengthy troubleshooting until they are solved, often distracting from actual data trends.

All days did not require troubleshooting, fortunately. Some days showed clear reproducibility trends across the various column chemistries. The two-injection series shown in Figure 31 and Figure 32, are both for four serial injections of MMA-d3 at $1 \mu \mathrm{g} / \mathrm{mL}$. The two column chemistries compared are C18 in Figure 31 and DH in 32. For both of these injection series, all method parameters are held constant, as described in Section 3.2.1. 


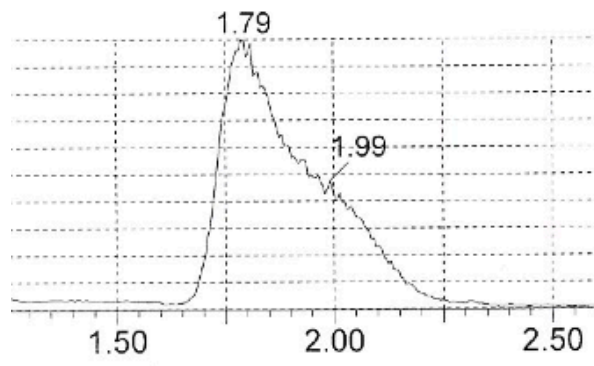

\section{4}
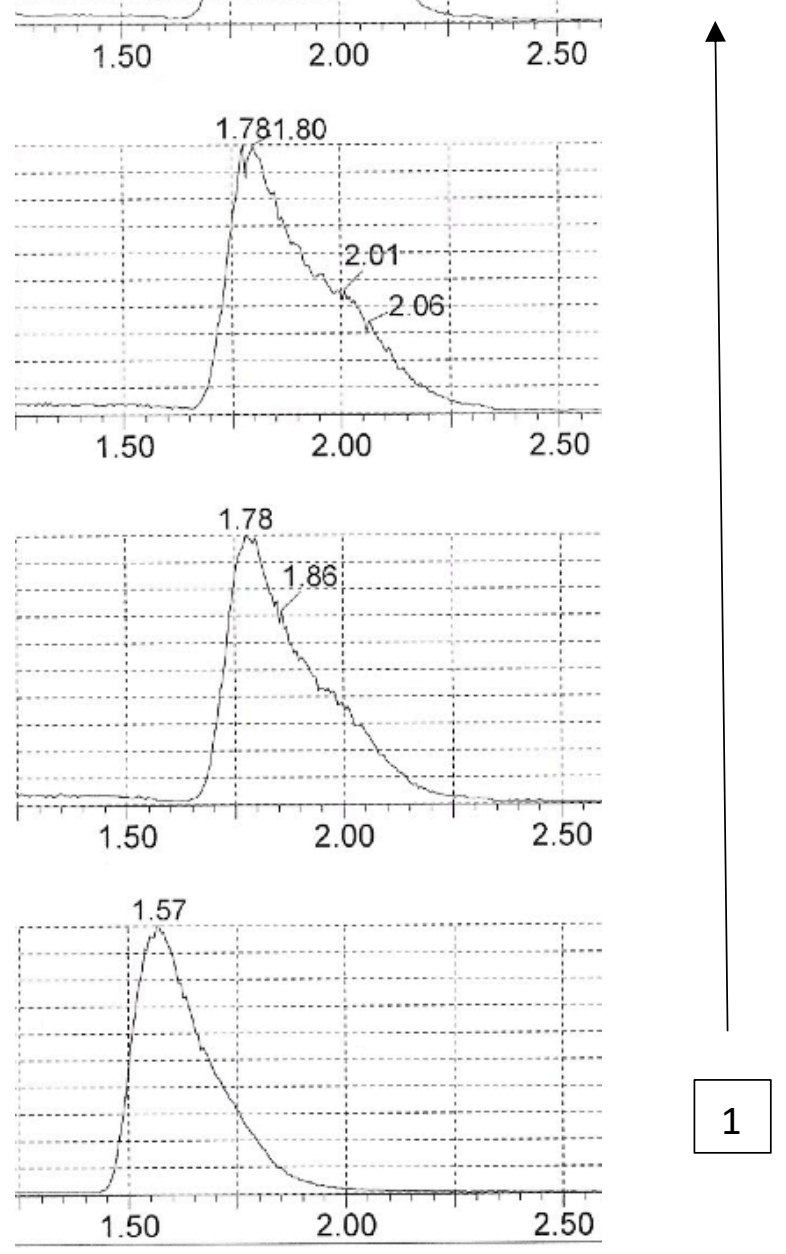

Figure 31. C18 repeat injection series. The chromatograms are shown in ascending order, with the lowest displayed injection 1 being the first and injection 4 being last and displayed at the top of the figure. The x-axis has the time displayed in minutes. All data shown were obtained on the same C18 column.

It can be seen in Figure 31 that the peak shape for MMA-d3 gets worse as the injections progress. The shoulder becomes more pronounced with each repeat injection, and there are other carryover issues as shown in Figure 33. 


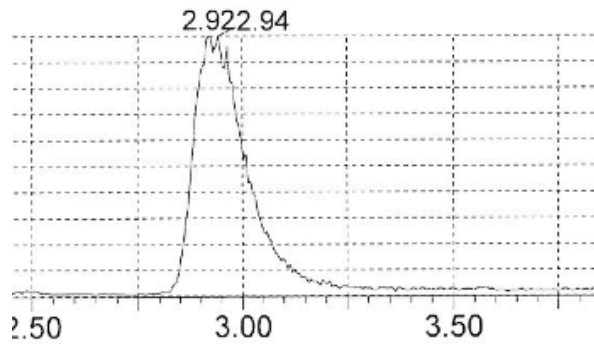

\section{4}
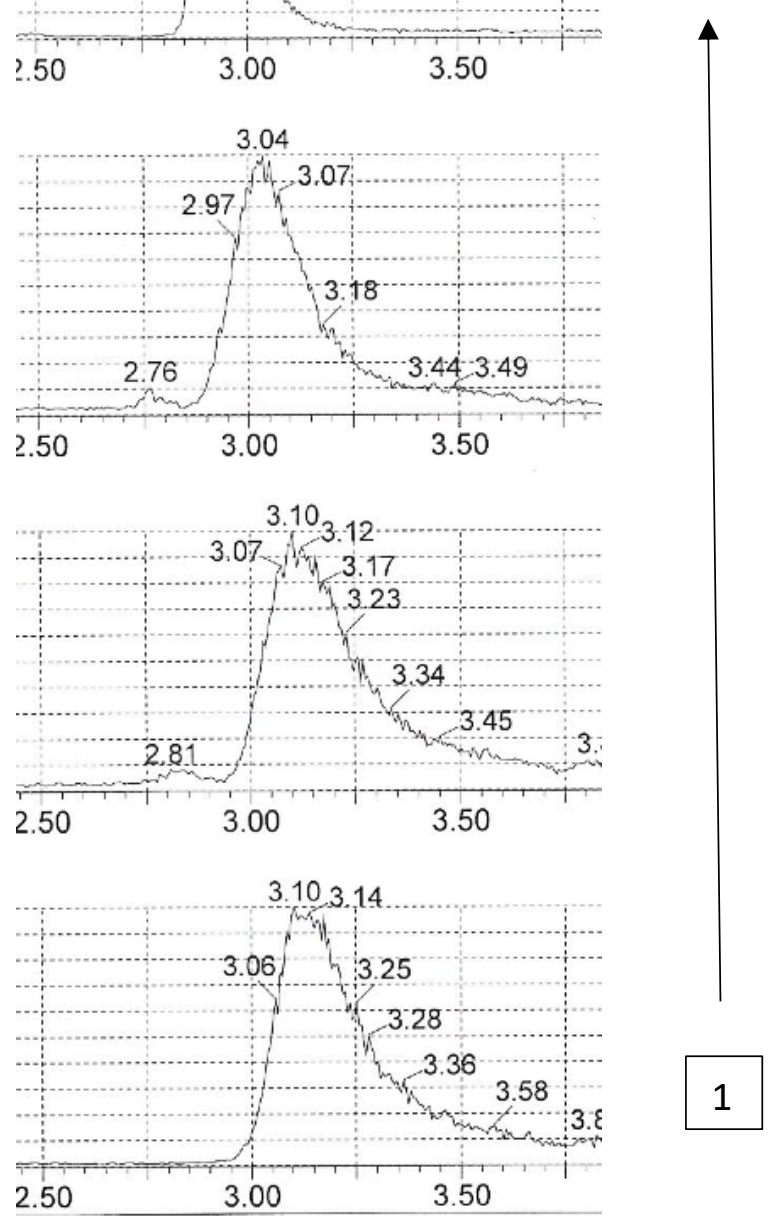

Figure 32. DH repeat injection series. The chromatograms are shown in ascending order, with the lowest displayed injection 1 being the first and injection 4 being last and displayed at the top of the figure. The $\mathrm{x}$-axis has the time displayed in minutes.

The initial peak shown in Figure 32 at the bottom labeled 1 is broader and tailing slightly, indicating the column's inadequate priming. However, by the fourth injection (top), peak shape is significantly improved, and there is no carryover evidence. The injection sequence that highlighted that carryover was an issue on the $\mathrm{C} 18$ column was 
injecting MMA four times, followed by injecting only MMA-d3 four times. It could be seen that MMA carried over, RT of 0.89 , into the first injection of MMA-d3 at a relatively high quantity indicating the need for additional column washes (Figure 33).

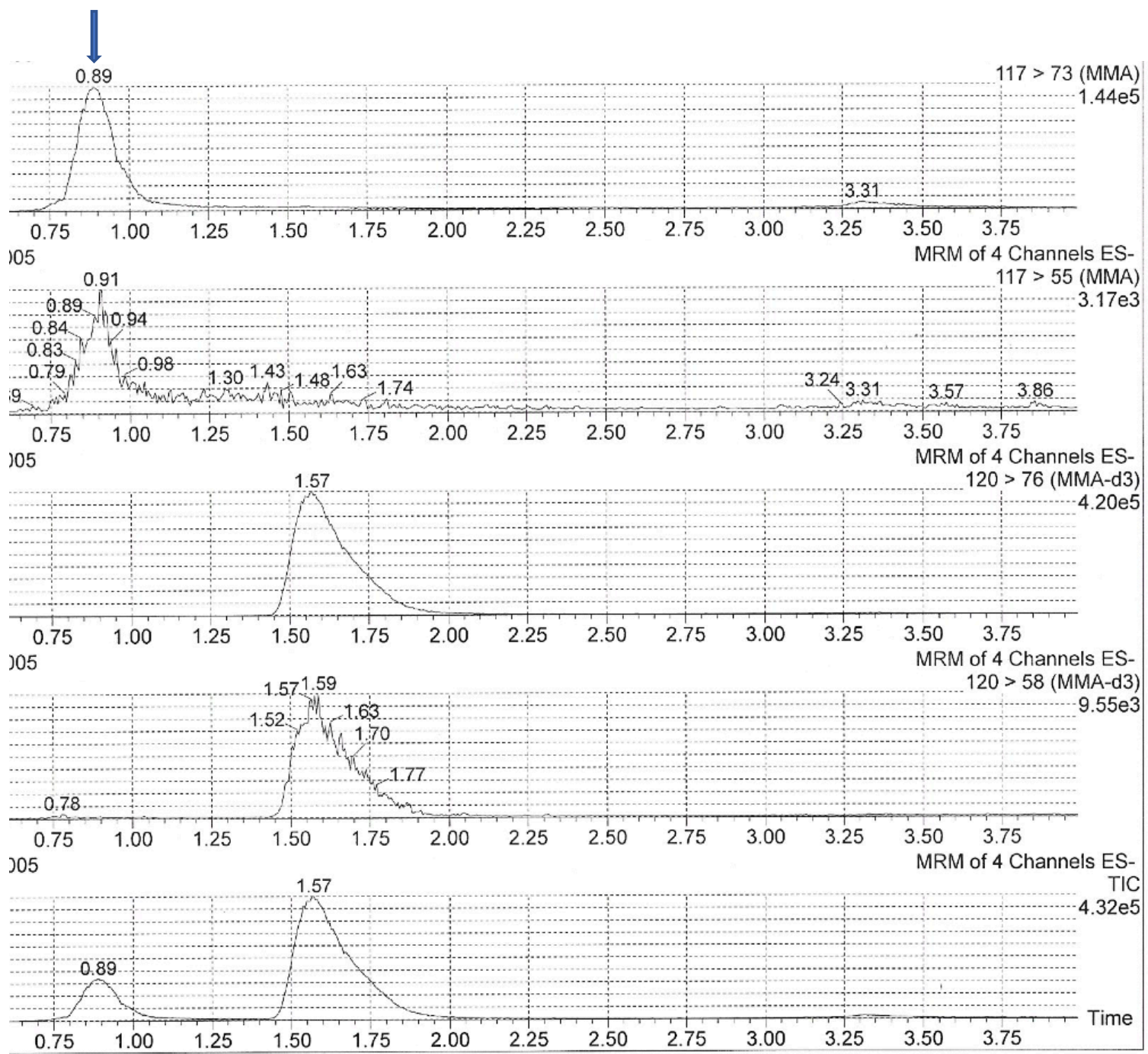

Figure 33. Carryover of MMA on C18 column. Displayed is a TIC in the bottom pane, with two peaks, one at 0.89 minutes and one at 1.57 minutes. The four panes above show MMA and MMA-d $3 \mathrm{~m} / \mathrm{z}$ transitions, respectively. The $\mathrm{x}$-axis displays the time in minutes.

It is observed that the MMA from the previous injection is still present on the column and elutes out at 0.89 minutes. While the MMA-d3 peak elutes out at 1.57 mins, as 
indicated by the $\mathrm{m} / \mathrm{z}$ transitions as displayed in Figure 36. The subsequent injections would show that the MMA peak at 0.89 minutes disappears, but a shoulder develops on MMA-d3 (Figure 31). This potential for carryover combined with poor reproducibility would require extra volumes of column wash, whether done as part of re-equilibration or as a blank injection every few samples, would prolong the run time and make for a less useful clinical method.

Ultimately, lengthy priming and fastidious cleaning of instrument components allowed for some successful days. However, many puzzling moments, highlight the inexperience with method development inherent in the chosen approach. The method arrived at is a preliminary method that has shown promise. However, there are still days where the reproducibility of retention time has proven unattainable, and no clear explanation can be given, across multiple columns. The miscibility of ethyl acetate, acetonitrile, and water is not ideal, and resulted in some unstable back pressures, even after lengthy primes. In Figure 34, the pressure trace of the binary solvent manager over 10 minutes is shown. After significant priming, the LC conditions for this image are $0.750 \mathrm{~mL} / \mathrm{min}$ ethyl acetate on a DH column. The delta values were low generally, $<10$, but occasionally showed significant deviations. The BSM pressure trace showed an upward trend at times and an erratic line at other times, not a stable level trace, as is expected when priming for initial injections. The data shown in Figure 34, top and bottom, were obtained on separate days but highlight the variability in instability witnessed during method development. 

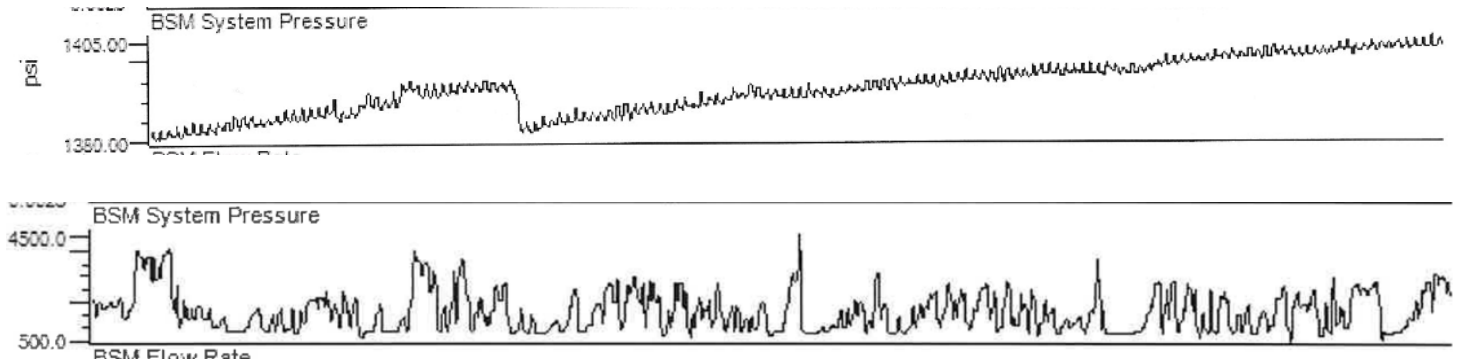

Figure 34. BSM back pressure issue with ethyl acetate prime. The y-axes are both PSI; the $\mathrm{x}$-axes are both the time in minutes with about ten minutes of data shown in each plot. The unstable system pressure displayed a wide range of trends and unpredictability.

Different hypotheses for this issue were discussed previously, and it is currently an ongoing issue with this method. Modifications have been made to the gradient and reequilibration parameters, yet this issue persists. It is possible that high salt concentrations on the column, combined with quick changes in MP composition to ethyl acetate resulted in salt solubility issues. It is also possible to run at elevated column temperatures or pre-mix some of the solvents like a 95:5 ethyl acetate:water loading MP may have helped the back pressure issues and resulted in more stable chromatography. There was a slight back pressure improvement when using a lower salt MP, such as $10 \mathrm{mM}$ vs. $100 \mathrm{mM}$ ammonium acetate. The lower salt MP resulted in more consistent retention times and will be discussed in section 3.2.4.

\subsubsection{Selectivity and Resolution}

Moving towards the ultimate goal of developing a clinical method that can separate and quantitate SA and MMA by LC-MS/MS ,several injections were performed across all three columns with a sample mixture of all three analytes. All three analytes were mixed in $\mathrm{MeOH}$ to a $1 \mu \mathrm{g} / \mathrm{mL}$ concentration and injected to measure selectivity and resolution values. The injections are displayed in Figures 35-37, with the TIC in the bottom pane, 
and the most robust transition for each compound shown in the three panes above that.

Figure 35 shows the data for the BEH-Amide column. Figure 36 shows the data for the C18 column. Figure 37 shows data for the DH column.

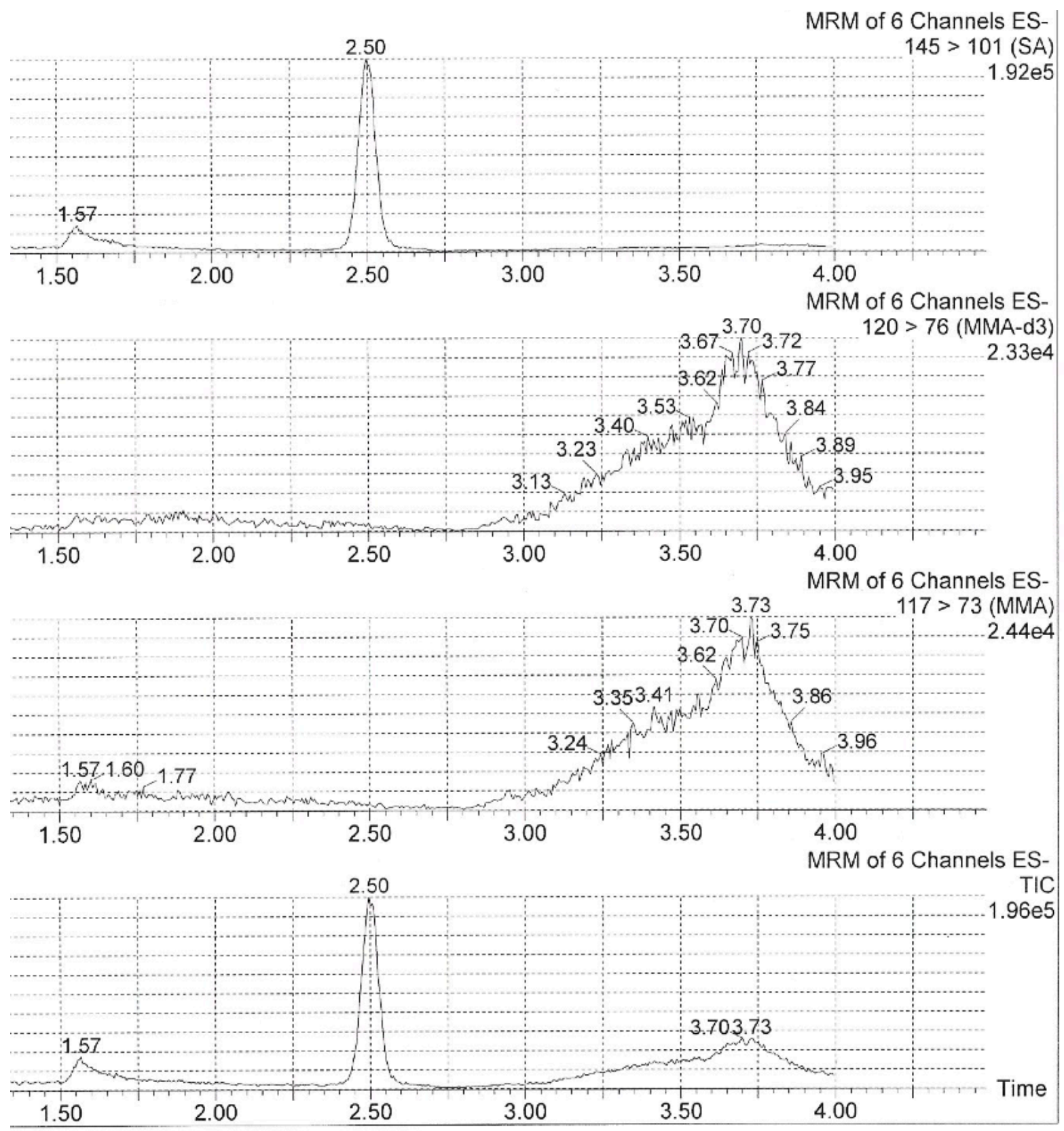

Figure 35. BEH-Amide all 3 analytes. One injection, TIC at the bottom, all three compounds shown by their dominant transitions above. The $\mathrm{x}$-axis is time in minutes. 
The SA* peak, represented by 2,2 dimethylsuccinic acid, is sharp by all three column chemistries, however, comparing the MMA and MMA-d3 peaks show a different picture. The peak shapes for MMA and MMA-d3 in Figure 35 are quite problematic, with a large fronting skew and clear shoulder. The selectivity and resolution values for the BEH-Amide column, based on this injection, are in Table 11.

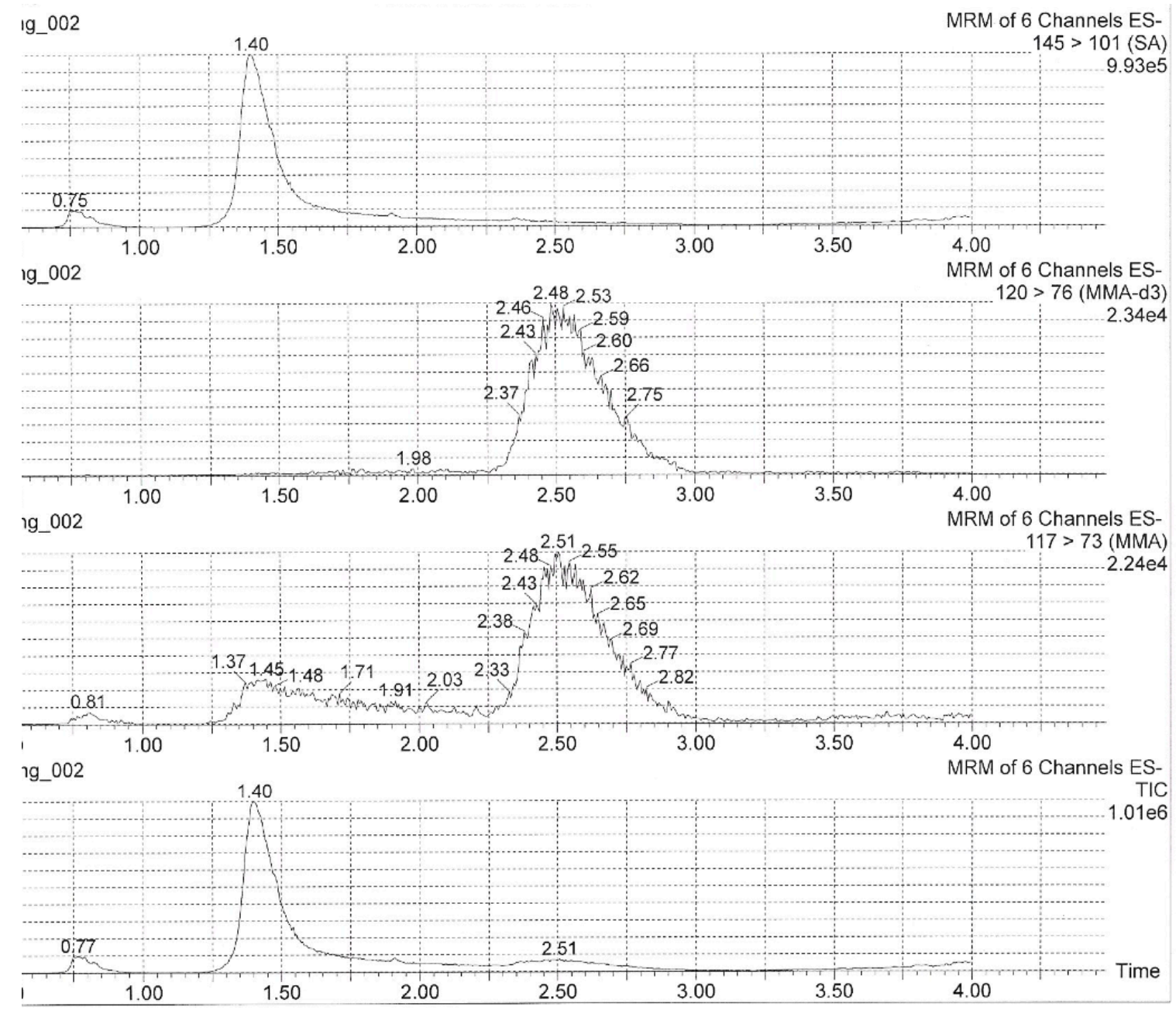

Figure 36. C18 all 3 analytes. One injection, TIC at the bottom, all three compounds shown by their dominant transition above. The $\mathrm{x}$-axis is time in minutes. 
Similar to Figure 35, SA* is a nice sharp peak in Figure 36 on a C18 column.

However, the peak shape for MMA and MMA-d3 has some improvements relative to the peak in Figure 35. The peak shapes for MMA and MMA-d3 are more symmetrical than on the BEH-Amide; however, they are incredibly broad, with a peak width substantially wider than seen in Figure 37. The selectivity and resolution values for the C18 column, based on this injection, are in Table 11. 


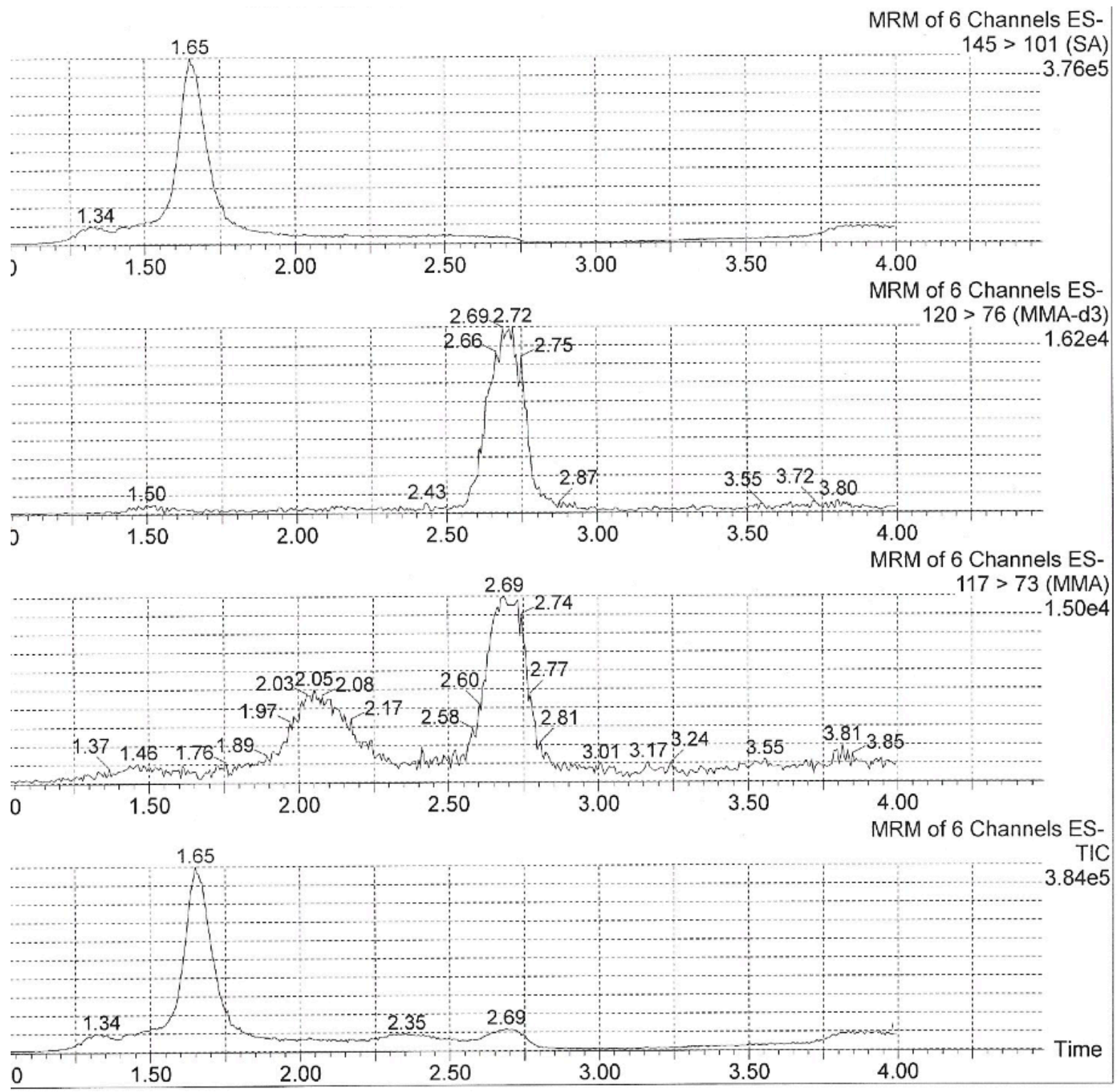

Figure 37. DH all 3 analytes. One injection, TIC at the bottom, all three compounds shown by their dominant transition above. X-axis is time in minutes. $100 \mathrm{mM}$ ammonium acetate in the MP.

Figure 37 shows a sharp SA* peak, just like in Figures 35 and 36. However, it also shows relatively sharp MMA and MMA-d3 peaks. There is a defect in the MMA peak in that it has a smaller peak eluting earlier that is not baseline resolved from MMA, similar to what is seen in Figure 36 for MMA on C18. This defect can be improved upon with 
more chromatographic optimization. It could likely be accounted for by modifying the integration parameters, as it is clearly a second peak and not a shoulder. The clear advantage that DH shows is in the peak width for MMA and MMA-d3, with the sharpest most symmetrical peak of all three column chemistries. The other results that stood out for this MP mixture on the DH column were the capacity factor $(k)$ and resolution values, which were quite good. The full summary of chromatographic results in these MP conditions across all three columns can be seen in Table 11 .

Table 11. Summary of Chromatographic Results Obtained with High Salt MP

\begin{tabular}{|c|c|c|c|c|c|c|c|c|}
\hline Column & $\mathrm{t}_{0}$ & $\mathrm{RT}_{\mathrm{SA}^{*}}$ & $\mathrm{RT}_{\mathrm{MMA}}$ & $k_{\mathrm{SA}^{*}}$ & $k_{\mathrm{MMA}}$ & $\alpha$ & $w_{1 / 2}-\mathrm{MMA}$ & $\mathrm{R}_{\mathrm{MMA}}$ \\
\hline BEH Amide & 1.5 & 2.5 & 3.73 & 0.66 & 1.48 & $\mathbf{2 . 2 4}$ & 0.43 & 1.69 \\
\hline XB-C18 & 0.75 & 1.4 & 2.51 & 0.87 & 0.97 & 1.11 & 0.16 & 0.45 \\
\hline $\mathrm{DH}$ & 0.57 & 1.65 & 2.69 & 1.89 & $\mathbf{3 . 7 2}$ & 1.96 & $\mathbf{0 . 1 3}$ & $\mathbf{4 . 7 0}$ \\
\hline
\end{tabular}
(all times listed in minutes)

It is interesting to note that while DH did show the highest capacity factor for MMA, the sharpest peak, and the best resolution, it did not achieve the best selectivity. The best selectivity was achieved by the BEH-amide; however, the column was twice as long, and the run would need to be extended to accommodate a clinical method on this length of the column. In addition, the peak shape would require significant troubleshooting as the width and fronting seen on the $\mathrm{BEH}$-amide are the cause of the lower resolution, despite having a high selectivity value. As expected, these analytes are not well suited for separation on a $\mathrm{C} 18$ column, and the data shows that. 
The data displayed is far from a method ready to put into production. However, there is promise for Cogent's DH as a viable stationary phase for the separation of the analytes SA and MMA. While the data is not entirely representative of the endogenous analyte mixture, where succinic acid, not 2,2 dimethylsuccinic acid, is likely to be more challenging to separate from MMA, the potential is obvious. To further examine if the DH column's retention exhibited an expected ANP-like behavior as opposed to a HILIC retention mechanism experiments varying salt concentration were undertaken and will be discussed in section 3.2.4.

\subsubsection{Buffer Salt Concentration to Verify ANP Retention Principles}

Once the preliminary peak shape and retention times were established with a high salt MP composition, the concentration and choice of buffer salt were varied to observe the effect on RT and peak shape. The expectation is that, for an acidic compound on a HILIC column, the presence of negatively charged silanols will repel the compound. By increasing the molar strength of the MP salt, counter ions are provided which weaken this repulsion and result in increased retention. ${ }^{33}$ In other words, if the column is retaining based on a HILIC retention mechanism the retention time will scale with molarity of the MP. In contrast, retention by an ANP mechanism follows the opposite trend, less salt results in greater retention. Now that the baseline RT has been established at a concentration of $100 \mathrm{mM}$ ammonium acetate, decreasing the salt concentration to $10 \mathrm{mM}$ and monitoring the effect on RT should provide some experimental evidence as to the type of retention mechanism the stationary phase exhibits. The baseline RT at "high salt" concentration can be seen in Figure 37. Keeping all other variables constant, including 
gradient and all solvents, the buffer additives were decreased from $100 \mathrm{mM}$ ammonium acetate with $1 \%$ formic acid (Figure 37 ) to $10 \mathrm{mM}$ ammonium acetate and $0.1 \%$ formic acid (Figure 38). 

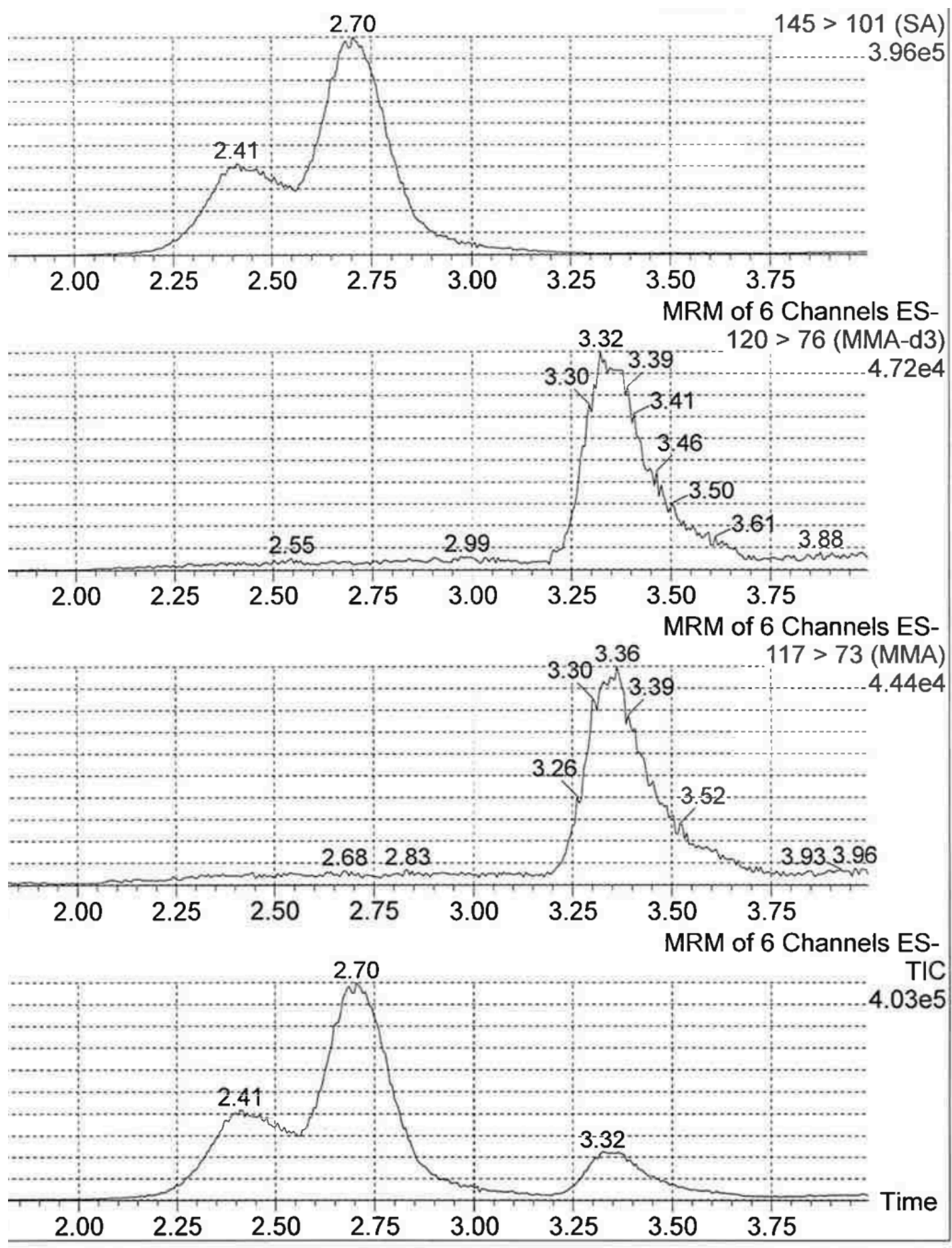

Figure 38. $10 \mathrm{mM}$ ammonium acetate $0.1 \%$ formic acid on $\mathrm{DH}$ by transition. One injection, TIC at the bottom, all three compounds shown by their dominant transition above. The $\mathrm{x}$-axis is time in minutes. $10 \mathrm{mM}$ ammonium acetate in the MP. 
In Figure 38, it can clearly be seen that the retention time increased substantially for both compounds, MMA, and SA*. However, it can also be seen that the peak shape for $\mathrm{SA}^{*}$ has deteriorated compared to prior conditions in Figure 37. One other interesting observation with the lower salt concentration was that the retention times became much more reproducible, likely due to the decrease in back pressure from miscibility issues with ethyl acetate and high salt concentrations. The reproducibility in peak shape and RT can be seen in Figure 39.
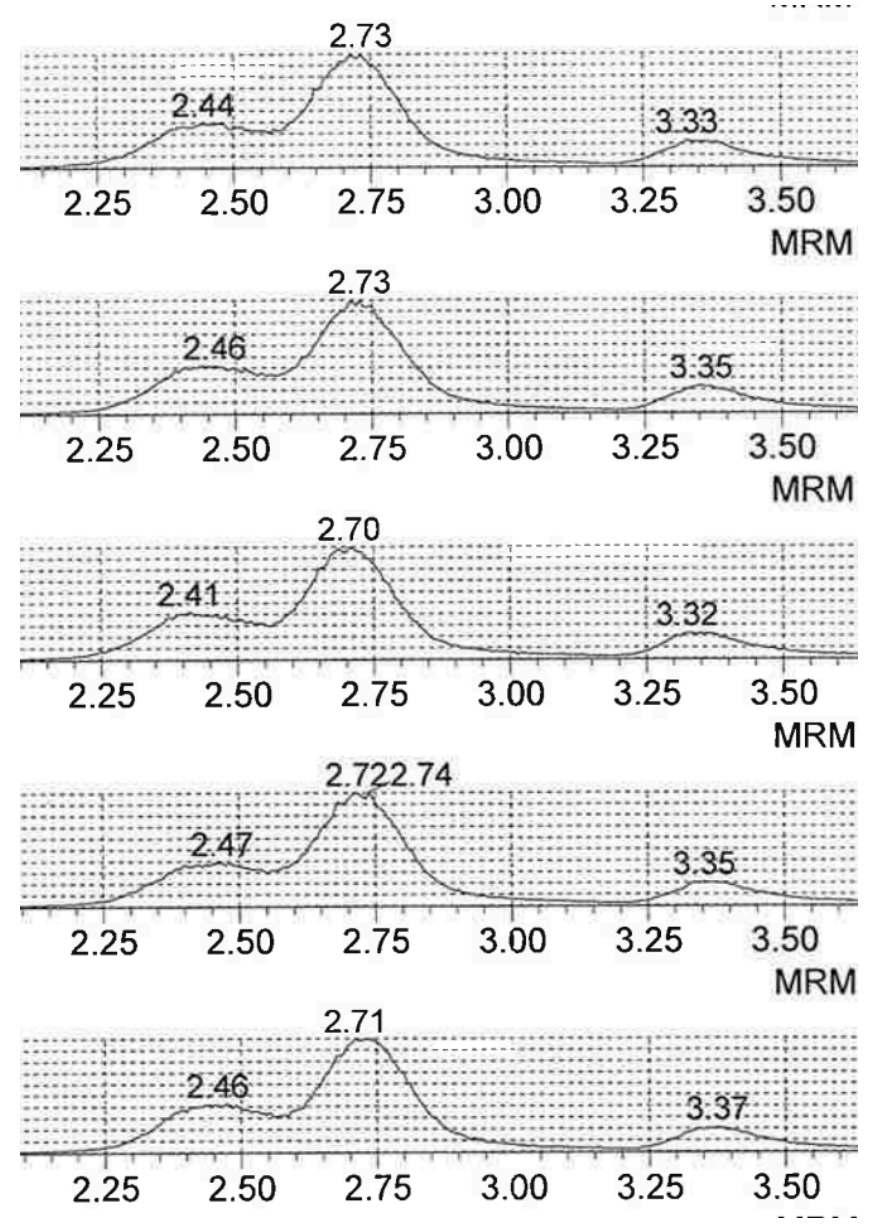

Figure 39. $10 \mathrm{mM}$ ammonium acetate $0.1 \%$ formic acid on DH repeats (TIC). Five sequential injections at "low-salt" concentration with ammonium acetate, first injection in the bottom pane, succeeding injections in ascending order. The $\mathrm{x}$-axis has time in minutes. 
The improvements realized by using low salt MP were numerous, increased retention, decreased back pressure leading to more reproducible chromatography and RT stability, as well as decreased cleaning requirements for the instrument. However, the peak shape for SA* definitely is worse with a clear shoulder and the peak width at half maximum for MMA-d3 increased. The peak width at half max increased from roughly 7.8 seconds to 9.6 seconds at this lower salt and formic acid concentration. To try to capitalize on the benefits of this lower salt concentration, yet improve upon the peak shape, a different buffer salt was attempted. In Figure 40, all chromatographic conditions were held constant relative to Figure 39, except ammonium formate was used instead of ammonium acetate. 


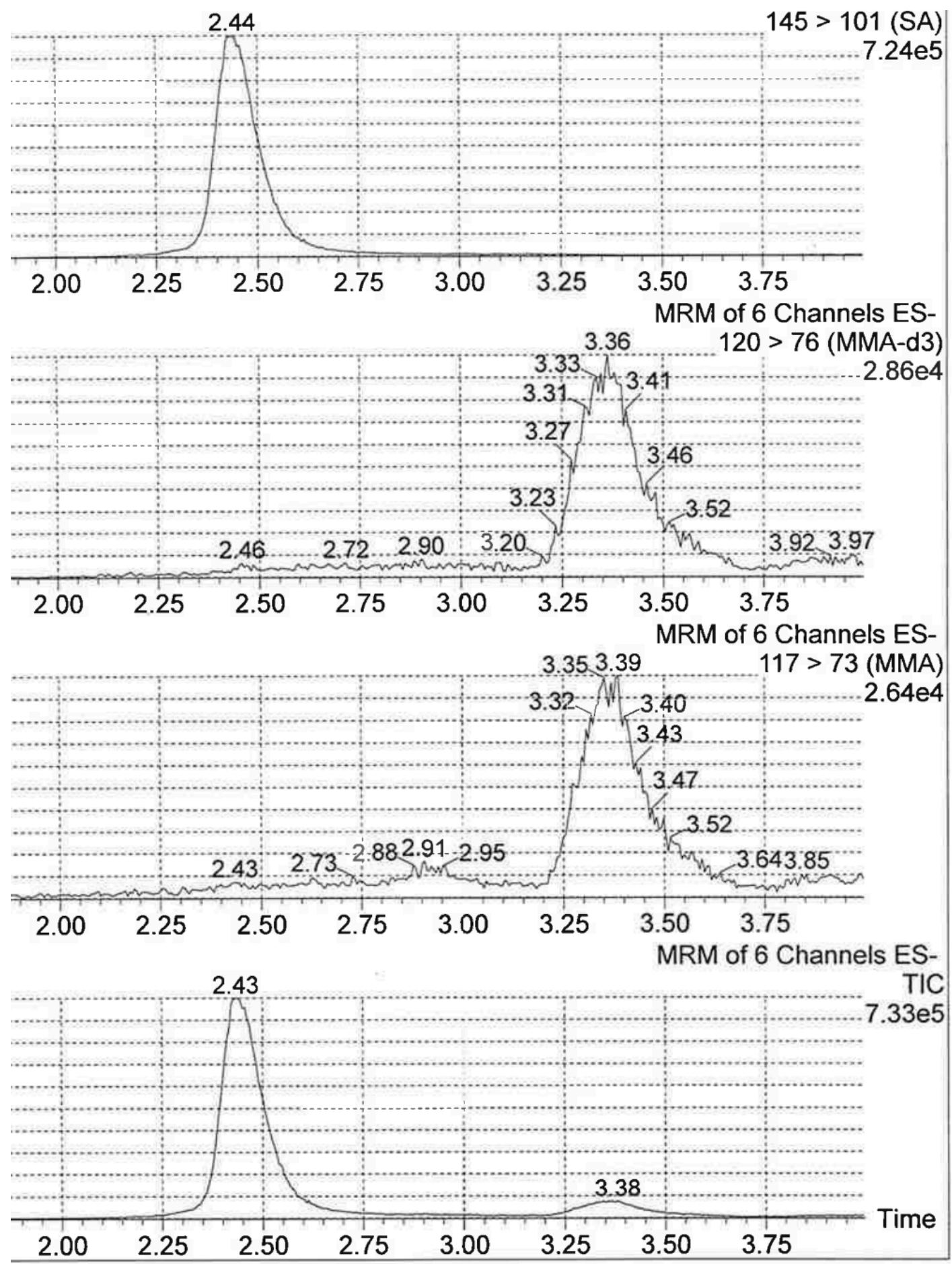

Figure 40. $10 \mathrm{mM}$ ammonium formate $0.1 \%$ formic acid on $\mathrm{DH}$ by transition. One injection, TIC at bottom, all three compounds shown by their dominant transition above. The $\mathrm{x}$-axis is time in minutes. $10 \mathrm{mM}$ ammonium formate in the MP. 
In Figure 40 ammonium formate is the new variable, once again at the same concentration as the "low salt" ammonium acetate in Figures 38-39. The ammonium formate yields interesting improvements to the peak shape of SA* at "low salt", while keeping the increased retention for MMA and MMA-d3 relative to the "high salt" injections observed in Figure 37. The resolution between MMA and SA* is actually improved by ammonium formate, however the peak width at half maximum for MMA-d3 is about 12 seconds. The lower salt concentration, this time ammonium formate, once again resulted in much more reproducibility in RT, as can be seen in Figure 41.

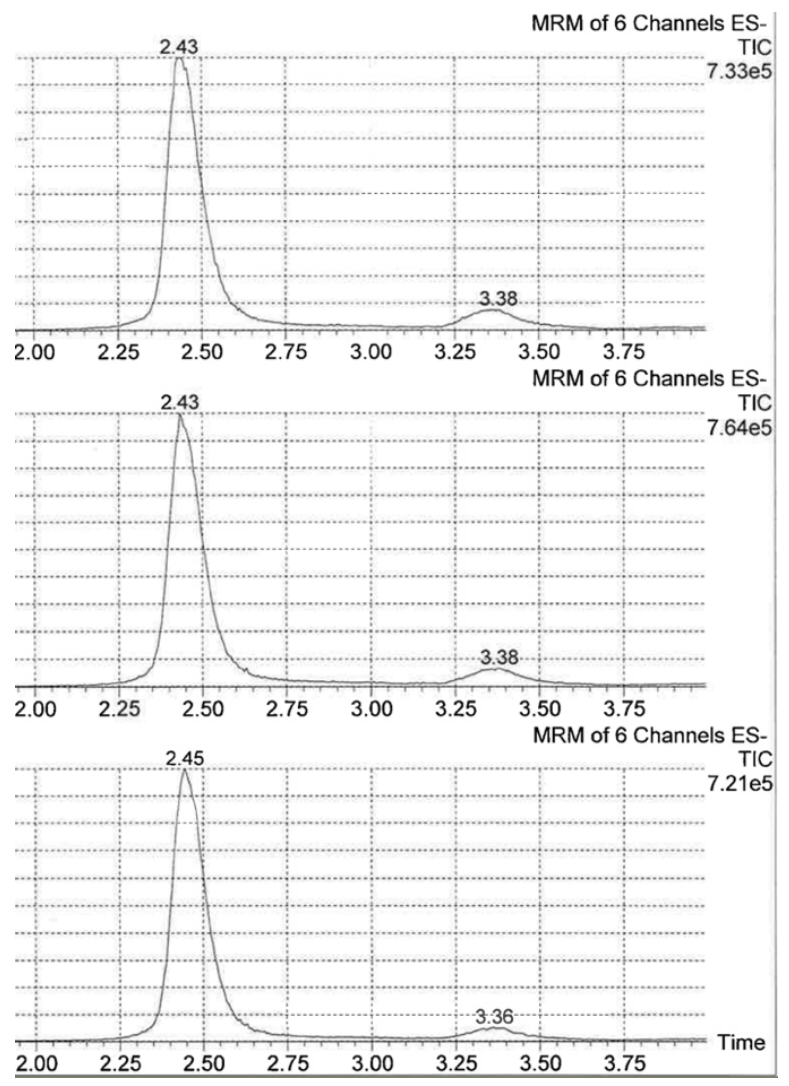

Figure 41. $10 \mathrm{mM}$ ammonium formate $0.1 \%$ formic acid on DH repeats (TIC). Repeatability of RT and peak shape look promising with low salt ammonium formate. The $\mathrm{x}$-axis has time in minutes. Three injections in series, lowest pane shows first injection. 
It should be noted that the signals for MMA and MMA-d3 were greater in the "high salt" situation, but this is likely due to the higher formic acid concentration that was paired with this high salt MP, rather than the salt itself. The various retention times, selectivity and resolution for the high salt versus low salt MP combinations on DH are summarized in Table 12.

Table 12. Varied MP Additive Concentration to Test ANP Retention Theory

\begin{tabular}{|c|c|c|c|c|}
\hline MP Composition & $\begin{array}{c}\text { RT (SA*, } \\
\text { MMA) (mins) }\end{array}$ & $\begin{array}{c}\text { Capacity } \\
\text { Factor } \\
\left(k_{\mathrm{MMA}}\right)\end{array}$ & Selectivity & Resolution \\
\hline $\begin{array}{c}\text { High Salt }- \\
100 \mathrm{mM} \text { ammonium acetate, } \\
1 \% \text { formic acid }\end{array}$ & $1.65,2.72$ & 3.72 & $\mathbf{1 . 9 6}$ & $\mathbf{4 . 7 0}$ \\
\hline $\begin{array}{c}\text { Low Salt }- \\
10 \mathrm{mM} \text { ammonium acetate, } \\
0.1 \% \text { formic acid }\end{array}$ & $2.70,3.36$ & $\mathbf{4 . 8 9}$ & 1.31 & 2.28 \\
\hline $\begin{array}{c}\text { Low Salt }- \\
10 \text { mM ammonium formate, } \\
0.1 \% \text { formic acid }\end{array}$ & $2.44,3.35$ & $\mathbf{4 . 8 8}$ & 1.49 & 2.99 \\
\hline
\end{tabular}

The results of this salt experiment on the DH column, confirm that the retention of these organic acids is inversely proportional to salt concentration in the MP, which agrees with established ANP retention mechanism theory. It would be interesting to try a slightly longer run time and an even lower salt concentration to see if the capacity factor would increase even more. While the capacity factor did increase it did so for both compounds which actually made the selectivity decrease. The decrease in selectivity in the low salt concentrations and a slightly broader peak, resulted in a decreased resolution as well. 


\section{CONCLUSION AND FUTURE WORK}

The purpose of this study was to perform preliminary experiments to guide the method development process for a quantitative LC-MS/MS assay for MMA in biological samples. The project proved immensely challenging and humbling due to the early results' inconsistency and the amount of time devoted to instrument troubleshooting, which made progress very gradual and non-linear. The two major aims of the project were the MS/MS method development and LC optimization. In addition to these major goals, LC optimization attempts were made to examine if the retention mechanism on DH is consistent with ANP theory.

The MS/MS method development demonstrated the importance of verifying that instrumentation is operating to specifications before beginning actual experiments. The mass accuracy, contamination and software hurdles made early experiments problematic, but these challenges eventually were overcome, and a valid mass calibration established for low-end ESI negative mode. Once the instrument was operating as expected, a combination of Intellistart and manual tuning successfully found multiple transitions per analyte and the MS/MS method was established. The transitions for MMA and MMA-d3 were verified by other groups in the literature, using ESI negative mode without derivatization as can be seen in Table 2 .

In addition, the parent ions were the expected $[\mathrm{M}-\mathrm{H}]^{-}$ions for these organic acids. There is still a good deal of room for improvement in terms of tuning parameters and optimizing the elution solvent MP composition to enhance the signal at the MS detector. The final $\mathrm{m} / \mathrm{z}$ transitions, cone voltages and collision energies established by this process 
can be seen in Table 8. Although a major challenge for a good portion of the project, in many ways the MS/MS method development was simpler than the ensuing LC optimization experiments.

The LC method development and optimization provided a number of challenges, many of which were due to the experimental design. The attempt to try to optimize one gradient and MP composition across three unique stationary phases, representing various separation modes, was an ill-fated endeavor. This approach is not recommended for no other reason than the amount of time spent priming the instrument. Although it is nice to be able to compare trends across columns and comment on the differences in selectivity, resolution and peak shape, compromises had to be made to choose solvents that would work across all three columns. A wiser approach would have been to choose one column chemistry and optimize, iterate and optimize again. The data obtained were often interesting, and each experiment brought questions to the forefront which guided future experiments. However, due to the attempts to make these adjustments and try each iteration across all three columns, experiments regularly were delayed. On the other hand, had the three columns and various chemistries not been screened it may not have been obvious which to choose.

Now that the three separation modes have been compared, a few things can be seen. There is potential to chromatographically separate MMA and SA, based on the data obtained with SA* This separation can be done without derivatization, and detection by MS/MS in ESI negative mode is a viable approach. The comparisons of RP, ANP, and HILIC for the chromatographic performance of MMA show the best peak shape, 
retention, and selectivity by ANP on the DH column with an atypical MP mixture. The C18 column using typical RPLC solvents showed no analyte retention, as expected, due to the polarity of the analytes. The experimentation with ethyl acetate as a loading MP and 80:20 acetonitrile:water as an elution MP with $100 \mathrm{mM}$ ammonium acetate and 1\% formic acid showed promising initial results across all three columns. The preliminary gradient and MP composition were only intended to be a starting point. However, with many successive iterations due to various challenges, the progress was not as rapid as expected. There are many unanswered questions and many variations that were untested, due to time, availability of solvents/reagents and need for troubleshooting.

When comparing all the data collected it is clear the best chromatographic performance for MMA is on the DH column, namely the high capacity factor, sharp peak and high resolution at high salt concentrations. Several trends were noted for various MP conditions that could assist future method development experiments for these compounds. For instance, at high salt concentrations the BEH-Amide showed the best selectivity among the conditions tested, with a value of 2.24. While the BEH-Amide did not perform well based on other metrics, it shows promise for separating these compounds that could likely be further refined with optimization experiments. Another notable trend was the increase in capacity factor at lower salt concentrations on the $\mathrm{DH}$ column, which suggests trying various salt concentrations, from $0.5 \mathrm{mM}$ up to $150 \mathrm{mM}$ at regular intervals, to see the true optimum in capacity factor and resolution. These lessons learned can be thought of as leads for future experimentation. In many circumstances, 
only extremes of various conditions could be tested, not an ideal way to optimize as promising trends may go unnoticed.

The most stable chromatography with a good capacity factor and resolution was at the lower salt, the $10 \mathrm{mM}$ ammonium formate or ammonium acetate and $0.1 \%$ formic acid elution combination for the elution MP. However, no attempts were made at different ammonium formate concentrations or at the same concentration but with various acid concentrations to improve the ionization efficiency. While RT was stable at the lower salt concentrations the peak shape was not quite as good, suggesting perhaps somewhere between $10 \mathrm{mM}$ and $100 \mathrm{mM}$ may be the ideal salt concentration. The peak shape when using a low salt concentration of ammonium formate is promising and warrants further investigation.

Even though there were issues with repeatability, the sharpest peak, and the best response were at high salt and acid concentration, $100 \mathrm{mM}$ ammonium acetate with $1 \%$ formic acid. This MP combination resulted in an MMA-d3 peak with a peak width at half maximum of 7.8 seconds, but the RT stability was not as good as what was observed with the low salt concentration or with acetonitrile:water as the loading MP. The increased concentration of formic acid was likely beneficial for the MS/MS response, yet no experiments were performed at low salt but with higher acid content, which would be interesting.

The salt concentration experiment provided some interesting commentary on the clear difference between ANP and HILIC retention mechanisms. The terms HILIC and ANP were often conflated in literature until the last decade as more seminal works have come 
out that delineate the two. Despite the recent published works which can point at clear differences in retention mechanism, there is not a large amount of experimental data or applications that clearly illustrate these theoretical principles. The salt concentration experiment did just that; lowered salt concentration resulted in increased retention. This inverse relationship makes a clear point that DH does not operate by a HILIC retention mechanism. The distinction is critical as it will guide future optimization experiments and provide theoretical backing to understand the results. The $\mathrm{LC}$ optimization is iterative, and future iterations should move away from the three-column approach and focus on the $\mathrm{DH}$ as the most promising column chemistry tested. Although the goal of a fully optimized LC-MS/MS method for the quantitative measurement of MMA was not realized, significant progress was made towards understanding MMA's chromatographic behavior on the $\mathrm{DH}$ column. 


\section{LITERATURE CITED}

(1) CLSI. Liquid Chromatography-Mass Spectrometry Methods; Approved Guideline. CLSI document C62-A. Wayne, PA: Clinical and Laboratory Standards Institute; 2014.

(2) Kushnir, M. M.; Komaromy-Hiller, G.; Shushan, B.; Urry, F. M.; Roberts, W. L. Analysis of Dicarboxylic Acids by Tandem Mass Spectrometry. High-Throughput Quantitative Measurement of Methylmalonic Acid in Serum, Plasma, and Urine. Clin. Chem. 2001, 47 (11), 1993-2002.

(3) Mayo Medical Laboratories https://www.mayocliniclabs.com/testcatalog/Clinical+and+Interpretive/80289.

(4) Baumgartner, M. R.; Hörster, F.; Dionisi-Vici, C.; Haliloglu, G.; Karall, D.; Chapman, K. A.; Huemer, M.; Hochuli, M.; Assoun, M.; Ballhausen, D.; Burlina, A.; Fowler, B.; Grünert, S. C.; Grünewald, S.; Honzik, T.; Merinero, B.; PérezCerdá, C.; Scholl-Bürgi, S.; Skovby, F.; Wijburg, F.; MacDonald, A.; Martinelli, D.; Sass, J. O.; Valayannopoulos, V.; Chakrapani, A. Proposed Guidelines for the Diagnosis and Management of Methylmalonic and Propionic Acidemia. Orphanet J. Rare Dis. 2014, 9 (1).

(5) Yuan, C.; Gabler, J.; El-Khoury, J. M.; Spatholt, R.; Wang, S. Highly Sensitive and Selective Measurement of Underivatized Methylmalonic Acid in Serum and Plasma by Liquid Chromatography-Tandem Mass Spectrometry. Anal. Bioanal. Chem. 2012, 404 (1), 133-140.

(6) California Department of Public Health. Newborn Screening Booklet. cdph.ca.gov/Programs/CFH/DGDS.

(7) Magera, M. J.; Helgeson, J. K.; Matern, D.; Rinaldo, P. Methylmalonic Acid Measured in Plasma and Urine by Stable-Isotope Dilution and Electrospray Tandem Mass Spectrometry. Clin. Chem. 2000, 46 (11), 1804-1810.

(8) Villani, G. R. D.; Gallo, G.; Scolamiero, E.; Salvatore, F.; Ruoppolo, M. Classical Organic Acidurias: Diagnosis and Pathogenesis. Clin. Exp. Med. 2016, 17 (3), $305-323$.

(9) Monostori, P.; Klinke, G.; Richter, S.; Baráth, Á.; Fingerhut, R.; Baumgartner, M. R.; Kölker, S.; Hoffmann, G. F.; Gramer, G.; Okun, J. G. Simultaneous Determination of 3-Hydroxypropionic Acid, Methylmalonic Acid and Methylcitric Acid in Dried Blood Spots: Second-Tier $\{L C\}-\{M S\} /\{M S\}$ Assay for Newborn Screening of Propionic Acidemia, Methylmalonic Acidemias and Combined Remethylation Disorders. $\{P L O S\}\{O N E\}$ 2017, 12 (9), e0184897. 
(10) Schroder, T. H.; Mattman, A.; Sinclair, G.; Vallance, H. D.; Lamers, Y. Reference Interval of Methylmalonic Acid Concentrations in Dried Blood Spots of Healthy, Term Newborns to Facilitate Neonatal Screening of Vitamin B12 Deficiency. Clin. Biochem. 2016, 49 (13-14), 973-978.

(11) Sowers, S. A Primer On Branched Chain Amino Acids. Lit. Educ. Diet. Suppl. 2009, No. 5, 1-6.

(12) Stabler, S. P.; Marcell, P. D.; Podell, E. R.; Allen, R. H.; Lindenbaum, J. Assay of Methylmalonic Acid in the Serum of Patients with Cobalamin Deficiency Using Capillary Gas Chromatography-Mass Spectrometry. J. Clin. Invest. 1986, 77 (5), 1606-1612.

(13) Hempen, C.; Wanschers, H.; van der Sluijs Veer, G. A Fast Liquid Chromatographic Tandem Mass Spectrometric Method for the Simultaneous Determination of Total Homocysteine and Methylmalonic Acid. Anal. Bioanal. Chem. 2008, 391 (1), 263-270.

(14) Stabler, S. P. Vitamin B12 Deficiency. N. Engl. J. Med. 2013, 368 (2), 149-160.

(15) Klee, G. G. Cobalamin and Folate Evaluation: Measurement of Methylmalonic Acid and Homocysteine vs Vitamin B12 and Folate. Clin. Chem. 2000, 46 (8), 1277-1283.

(16) Pedersen, T. L.; Keyes, W. R.; Shahab-Ferdows, S.; Allen, L. H.; Newman, J. W. Methylmalonic Acid Quantification in Low Serum Volumes by UPLC-MS/MS. $J$. Chromatogr. B 2011, 879 (19), 1502-1506.

(17) Burtis, Carl A.; Bruns, D. E. Tietz Fundamentals of Clinical Chemistry and Molecular Diagnostics, Seventh.; Sawyer, B. G., Ed.; Elsevier: St. Louis, Missouri, 2015.

(18) Mineva, E. M.; Zhang, M.; Rabinowitz, D. J.; Phinney, K. W.; Pfeiffer, C. M. An LC-MS/MS Method for Serum Methylmalonic Acid Suitable for Monitoring Vitamin B12 Status in Population Surveys. Anal. Bioanal. Chem. 2015, 407 (11), 2955-2964.

(19) Thorpe, S. J.; Heath, A.; Blackmore, S.; Lee, A.; Hamilton, M.; O'Broin, S.; Nelson, B. C.; Pfeiffer, C. International Standard for Serum Vitamin B12 and Serum Folate: International Collaborative Study to Evaluate a Batch of Lyophilised Serum for B12 and Folate Content. Clin. Chem. Lab. Med. 2007, 45 (3). 
(20) Lide, D. Dissociation Constants of Organic Acids and Bases. CRC Handb. Chem. physics. 2005, 42-51.

(21) Hoofnagle, A. N.; Wener, M. H. The Fundamental Flaws of Immunoassays and Potential Solutions Using Tandem Mass Spectrometry. J Immunol Methods. 2009, 347 (1-2):3-11.

(22) Jannetto, P. J.; Fitzgerald, R. L. Effective Use of Mass Spectrometry in the Clinical Laboratory. Clinical Chemistry. 2016, pp 92-98.

(23) Ettre, L. S.; Sakodynskii, K. I. M. S. Tswett and the Discovery of Chromatography I: Early Work (1899-1903). Chromatographia 1993, 35 (3), 223-231.

(24) Rimmer, C. A. Lloyd R. Snyder, Joseph J. Kirkland, John W. Dolan: Introduction to Modern Liquid Chromatography, 3rd Ed. Anal. Bioanal. Chem. 2010, 399 (5), 1809-1810.

(25) Maslow, A. H. The Psychology of Science: A Reconnaissance; Harper \& Row: New York, 1966.

(26) Rappold, B. A.; Grant, R. P. HILIC-MS/MS Method Development for Targeted Quantitation of Metabolites: Practical Considerations from a Clinical Diagnostic Perspective. J. Sep. Sci. 2011, 34 (24), 3527-3537.

(27) Hemstrom, P.; Irgum, K. Hydrophilic Interaction Chromatography; 2006; Vol. 29.

(28) Pesek, J. J.; Boysen, R. I.; Hearn, M. T. W.; Matyska, M. T. Hydride-Based HPLC Stationary Phases: A Rapidly Evolving Technology for the Development of New Bio-Analytical Methods. Anal. Methods 2013, No. January 2013, 1-100.

(29) Jandera, P.; Janás, P. Recent Advances in Stationary Phases and Understanding of Retention in Hydrophilic Interaction Chromatography. A Review. Anal. Chim. Acta. 2017, 967, 12-32.

(30) Rodríguez-Gonzalo, E.; García-Gómez, D. Hydrophilic Interaction Chromatography: Current Trends and Applications, 3rd ed.; Elsevier Inc., 2018.

(31) Jandera, P.; Hájek, T. Mobile Phase Effects on the Retention on Polar Columns with Special Attention to the Dual Hydrophilic Interaction-Reversed-Phase Liquid Chromatography Mechanism, a Review. J. Sep. Sci. 2018, 41 (1), 145-162. 
(32) Pesek, J. J.; Matyska, M. T. A New Approach to Bioanalysis: Aqueous NormalPhase Chromatography with Silica Hydride Stationary Phases. Bioanalysis 2012, 4 (7), 845-853.

(33) Grumbach, Eric S.; Fountain, K. J. Comprehensive Guide to HILIC: Hydrophilic Interaction Chromatography; Waters Corporation: Milford, Massachusetts, 2010.

(34) Watanabe, S. Synthesis and Evaluation of Novel Silica Hydride-Based Stationary Phases for Bioanalytical Applications. 2019.

(35) Soukup, J.; Jandera, P. Adsorption of Water from Aqueous Acetonitrile on SilicaBased Stationary Phases in Aqueous Normal-Phase Liquid Chromatography. $J$. Chromatogr. A 2014, 1374, 102-111.

(36) Griffiths, J. A Brief History of Mass Spectrometry. Analytical Chemistry. 2008.

(37) Greaves, J; Roboz, J. Mass Spectrometry for the Novice; CRC Press: Boca Raton, Florida, 2014.

(38) Dahl, P. F. A History of JJ Thomson's Electron: Flash of the Cathode Rays; Institue of Physics Publishing: London, Engladnd, 1997.

(39) Hoofnagle, A. N.; Wener, M. H. The Fundamental Flaws of Immunoassays and Potential Solutions Using Tandem Mass Spectrometry. J. Immunol. Methods 2009, 347 (1-2), 3-11.

(40) Yost, Richard A.; Enke, C. G. Triple Quadrupole Mass Spectrometry for Direct Mixture Analysis and Structure Elucidation; 1979.

(41) Grant, Russell P.; Crawford, M. LC-MSMS 301: Development and Validation of Quantitative LC-MS/MS Assays for Use in Clinical Diagnostics; Elsevier: Palm Springs, California, 2018.

(42) LITTLE, J.; WEMPE, M.; BUCHANAN, C. Liquid Chromatography-mass Spectrometry/Mass Spectrometry Method Development for Drug Metabolism Studies: Examining Lipid Matrix Ionization Effects in Plasma. J. Chromatogr. B 2006, 833 (2), 219-230.

(43) Windelberg, A.; A trrseth, O.; Kvalheim, G.; Ueland, P. M. Automated Assay for the Determination of Methylmalonic Acid, Total Homocysteine, and Related Amino Acids in Human Serum or Plasma by Means of Methylchloroformate Derivatization and Gas Chromatography-Mass Spectrometry. Clin. Chem. 2005, 51 (11), 2103-2109. 
(44) Keyfi, F.; Varatesh, A. Development and Validation of GC-FID Method for Diagnosis of Methylmalonic Acidemia. Rep. Biochem. Mol. Biol. 2016, 4 (2).

(45) Mineva, E. M.; Zhang, M.; Rabinowitz, D. J.; Phinney, K. W.; Pfeiffer, C. M. An $\{L C\}-\{$ MS $\} /\{$ MS $\}$ Method for Serum Methylmalonic Acid Suitable for Monitoring Vitamin B12 Status in Population Surveys. Anal. Bioanal. Chem. 2014, 407 (11), 2955-2964.

(46) Hempen, C.; Wanschers, H.; Van Der Sluijs Veer, G. A Fast Liquid Chromatographic Tandem Mass Spectrometric Method for the Simultaneous Determination of Total Homocysteine and Methylmalonic Acid. Anal. Bioanal. Chem. 2008, 391 (1), 263-270.

(47) Lo, S.-Y.; Gordon, C.; Sadilkova, K.; Jack, R. M.; Dickerson, J. A. Quantifying $\{$ MMA $\}$ by $\{\mathrm{SLE}\}\{\mathrm{LC}\}-\{\mathrm{MS}\} /\{\mathrm{MS}\}$ : Unexpected Challenges in Assay Development. Clin. Biochem. 2016, 49 (13-14), 967-972.

(48) Lakso, H.-A.; Appelblad, P.; Schneede, J. Quantification of Methylmalonic Acid in Human Plasma with Hydrophilic Interaction Liquid Chromatography Separation and Mass Spectrometric Detection. Clin. Chem. 2008, 54 (12), 2028-2035.

(49) Sriboonvorakul, N.; Leepipatpiboon, N.; Dondorp, A. M.; Pouplin, T.; White, N. J.; Tarning, J.; Lindegardh, N. Liquid Chromatographic-mass Spectrometric Method for Simultaneous Determination of Small Organic Acids Potentially Contributing to Acidosis in Severe Malaria. J. Chromatogr. B 2013, 941, 116-122.

(50) Pesek, J. J.; Matyska, M. T.; Fischer, S. M.; Sana, T. R. Analysis of Hydrophilic Metabolites by High-Performance Liquid Chromatography-Mass Spectrometry Using a Silica Hydride-Based Stationary Phase. J. Chromatogr. A 2008, 1204 (1).

(51) Waters MS Primer: Quantitation and Calibration https://www.waters.com/waters/en_US/Quantitation-and-Calibration/.

(52) Reichardt, C. Solvents and Solvent Effects in Organic Chemistry, 3rd ed.; WileyVCH Publishers, 2003. 